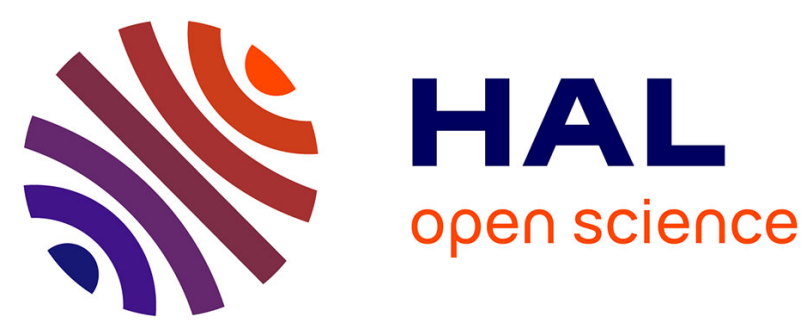

\title{
Toxicity and remediation of pharmaceuticals and pesticides using metal oxides and carbon nanomaterials
}

Zari Fallah, Ehsan Nazarzadeh Zare, Matineh Ghomi, Farhad Ahmadijokani, Majed Amini, Mahmood Tajbakhsh, Mohammad Arjmand, Gaurav Sharma, Hamna Ali, Awais Ahmad, et al.

\section{To cite this version:}

Zari Fallah, Ehsan Nazarzadeh Zare, Matineh Ghomi, Farhad Ahmadijokani, Majed Amini, et al.. Toxicity and remediation of pharmaceuticals and pesticides using metal oxides and carbon nanomaterials. Chemosphere, 2021, 275, pp.1-37. 10.1016/j.chemosphere.2021.130055 . hal-03170433

\section{HAL Id: hal-03170433 https://hal.science/hal-03170433}

Submitted on 16 Mar 2021

HAL is a multi-disciplinary open access archive for the deposit and dissemination of scientific research documents, whether they are published or not. The documents may come from teaching and research institutions in France or abroad, or from public or private research centers.
L'archive ouverte pluridisciplinaire HAL, est destinée au dépôt et à la diffusion de documents scientifiques de niveau recherche, publiés ou non, émanant des établissements d'enseignement et de recherche français ou étrangers, des laboratoires publics ou privés. 
Review

\title{
Toxicity and remediation of pharmaceuticals and pesticides using metal oxides and carbon nanomaterials
}

\author{
Zari Fallah a, Ehsan Nazarzadeh Zare ${ }^{\text {b, * }}$, Matineh Ghomi ${ }^{\text {b }}$, Farhad Ahmadijokani ${ }^{\text {c }}$ \\ Majed Amini ${ }^{\text {c }}$, Mahmood Tajbakhsh ${ }^{a}$, Mohammad Arjmand ${ }^{\text {c}}$, Gaurav Sharma d, m,

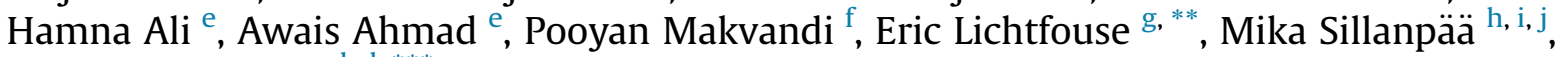 \\ Rajender S. Varma ${ }^{k, 1, * * *}$
}

a Faculty of Chemistry, University of Mazandaran, Babolsar, 47416-95447, Iran

b School of Chemistry, Damghan University, Damghan, 36716-41167, Iran

c School of Engineering, University of British Columbia, Kelowna, BC, V1V 1V7, Canada

${ }^{\mathrm{d}}$ College of Materials Science and Engineering, Shenzhen Key Laboratory of Polymer Science and Technology, Guangdong Research Center for Interfacial Engineering of Functional Materials, Nanshan District Key Lab. for Biopolymers and Safety Evaluation, Shenzhen University, Shenzhen, 518055, PR China

e Department of Chemistry, The University of Lahore, Lahore, 54590, Pakistan

${ }^{\mathrm{f}}$ Center for Micro-BioRobotics, Istituto Italiano di Tecnologia (IIT), Viale R. Piaggio 34, 56025, Pontedera, Pisa, Italy

${ }^{g}$ Aix-Marseille University, CNRS, IRD, INRA, Coll France, CEREGE, 13100, Aix en Provence, France

${ }^{\mathrm{h}}$ Institute of Research and Development, Duy Tan University, Da Nang, 550000, Viet Nam

${ }^{i}$ Faculty of Environment and Chemical Engineering, Duy Tan University, Da Nang, 550000, Viet Nam

${ }^{\mathrm{j}}$ Department of Chemical Engineering, School of Mining, Metallurgy and Chemical Engineering, University of Johannesburg, P. O. Box 17011, Doornfontein, 2028, South Africa

${ }^{\mathrm{k}}$ Chemical Methods and Treatment Branch, Water Infrastructure Division, Center for Environmental Solutions and Emergency Response, U. S.

Environmental Protection Agency, 26 West Martin Luther King Drive, Cincinnati, OH, 45268, USA

${ }^{1}$ Regional Center of Advanced Technologies and Materials, Department of Physical Chemistry, Faculty of Science, Palacky University, Š lechtitelu 27, 783 71,

Olomouc, Czech Republic

${ }^{\mathrm{m}}$ International Research Centre of Nanotechnology for Himalayan Sustainability (IRCNHS), Shoolini University, Solan 173212, Himachal Pradesh, India

Abbreviations: ClP, chlorophenol; CMs, carbamates; CNTs, carbon nanotubes; CPF, chlorpyrifos; CYP450, Cytochrome P450; 2D, two-dimensional; 2,4-D, 2,4-

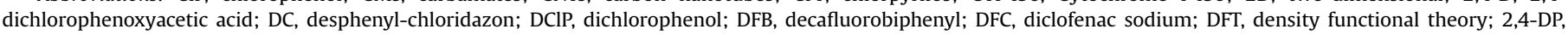

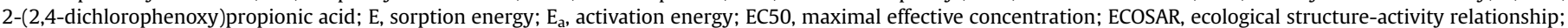

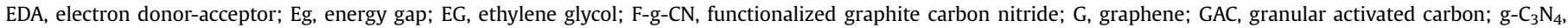

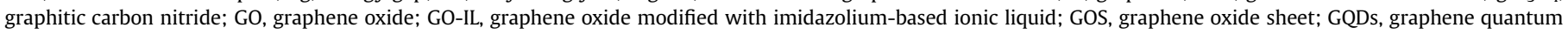

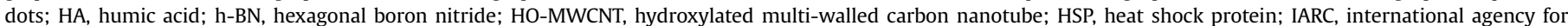

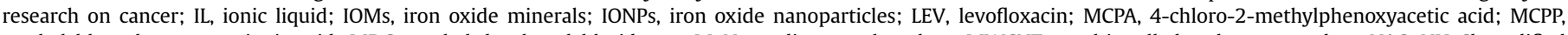

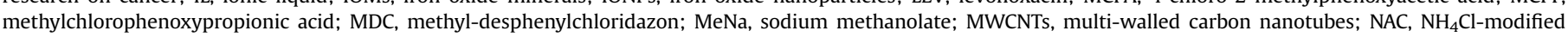

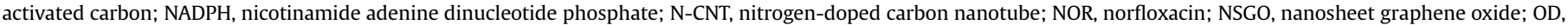

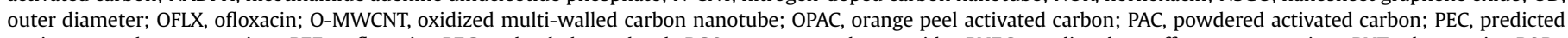

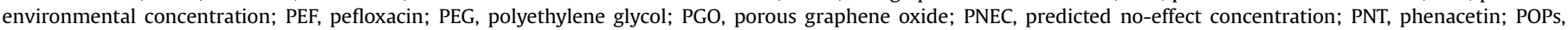

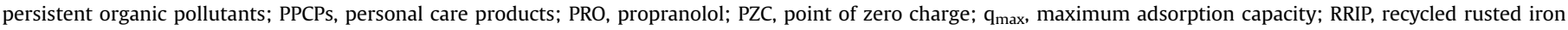

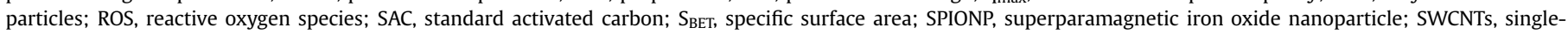

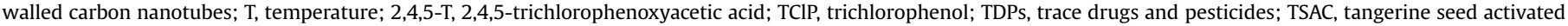
carbon; WHO, world health organization; WSAC, walnut shell activated carbon.

* Corresponding author.

** Corresponding author.

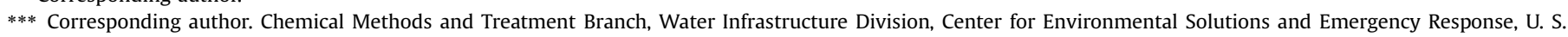
Environmental Protection Agency, 26 West Martin Luther King Drive, Cincinnati, OH, 45268, USA.

E-mail addresses: ehsan.nazarzadehzare@gmail.com, e.nazarzadeh@du.ac.ir (E.N. Zare), eric.lichtfouse@inrae.fr (E. Lichtfouse), Varma.Rajender@epa.gov (R.S. Varma). 
- Water contamination by drugs/pesticides led to environmental issues.

- Metal oxides/carbon-based sorbents used for drugs/pesticides removal.

- Residual drugs in the human body cause serious issues e.g., genetic disorders, etc.

- Presence of pesticides in the body leads to Parkinson's disease and breast cancer.

- Chemisorption and physisorption are main mechanisms for drugs and pesticides removal.

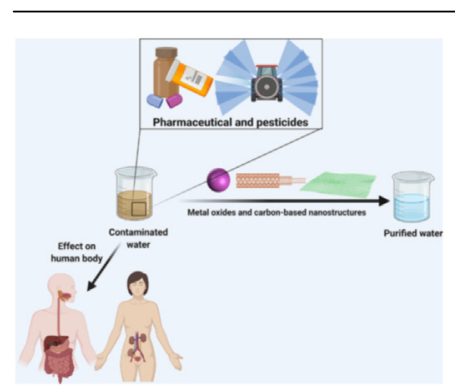

\begin{abstract}
A B S T R A C T
The worldwide development of agriculture and industry has resulted in contamination of water bodies by pharmaceuticals, pesticides and other xenobiotics. Even at trace levels of few micrograms per liter in waters, these contaminants induce public health and environmental issues, thus calling for efficient removal methods such as adsorption. Recent adsorption techniques for wastewater treatment involve metal oxide compounds, e.g. $\mathrm{Fe}_{2} \mathrm{O}_{3}, \mathrm{ZnO}, \mathrm{Al}_{2} \mathrm{O}_{3}$ and $\mathrm{ZnO}-\mathrm{MgO}$, and carbon-based materials such as graphene oxide, activated carbon, carbon nanotubes, and carbon/graphene quantum dots. Here, the small size of metal oxides and the presence various functional groups has allowed higher adsorption efficiencies. Moreover, carbon-based adsorbents exhibit unique properties such as high surface area, high porosity, easy functionalization, low price, and high surface reactivity. Here we review the cytotoxic effects of pharmaceutical drugs and pesticides in terms of human risk and ecotoxicology. We also present remediation techniques involving adsorption on metal oxides and carbon-based materials.
\end{abstract}

Keywords:

Human risk

Pharmaceutical

Pesticides

Metal oxides

Carbon nanostructures

\section{Contents}

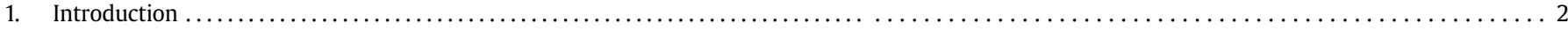

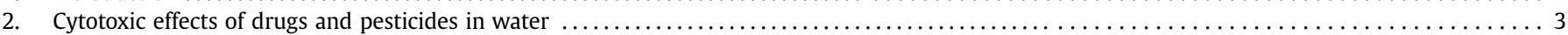

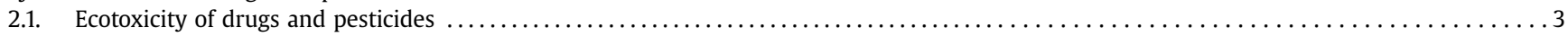

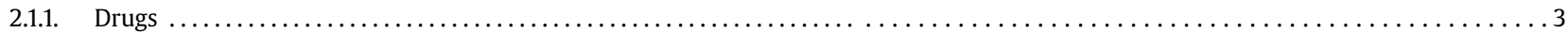

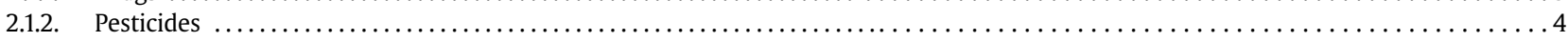

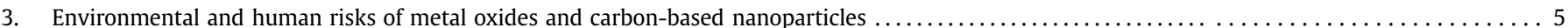

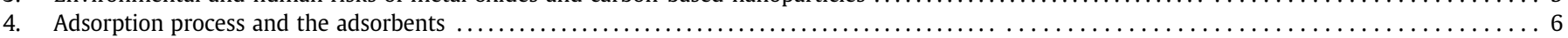

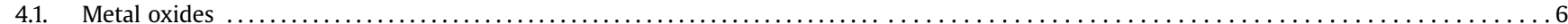

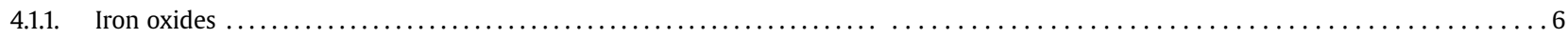

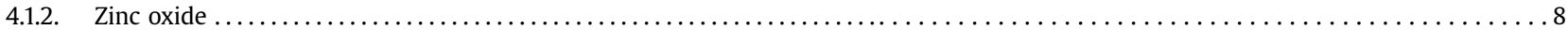

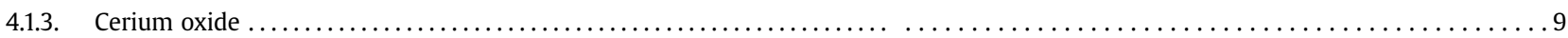

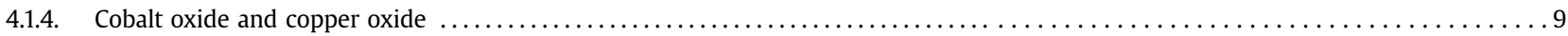

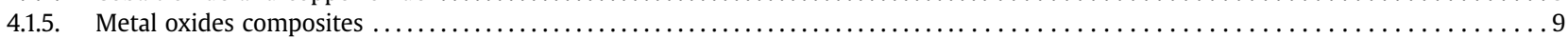

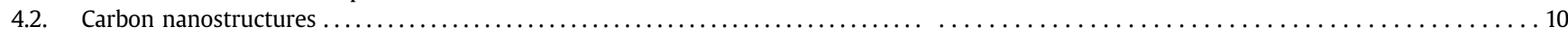

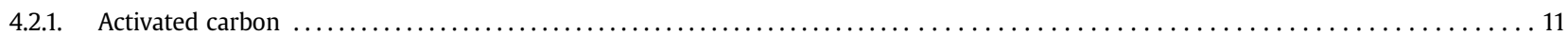

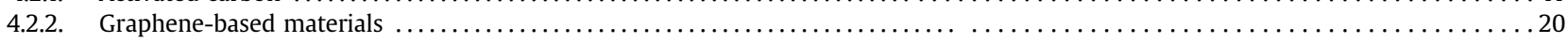

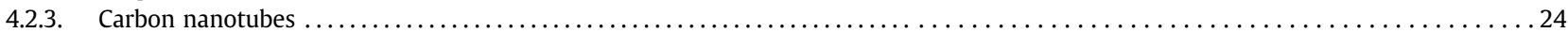

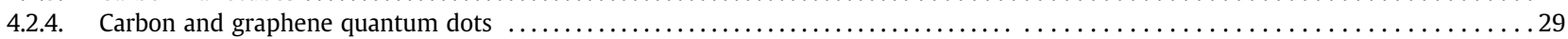

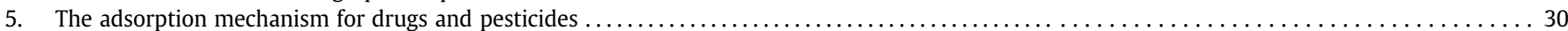

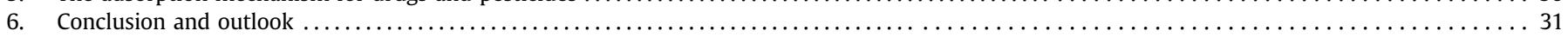

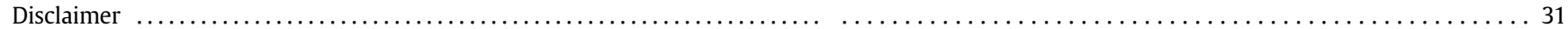

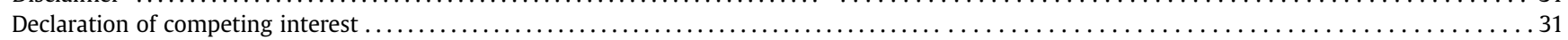

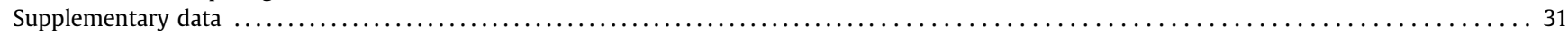

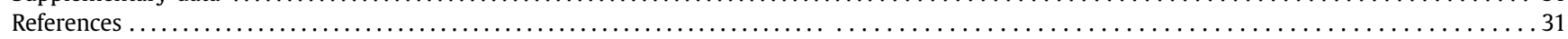

\section{Introduction}

Population growth, overexploitation of finite water supplies, and contamination by human, agricultural and industrial practices will lead to a future water shortage if advanced techniques are not rapidly designed to clean water (Márquez et al., 2014). By 2050, water and food demand are predicted to rise by $50 \%$ and $70 \%$, 
respectively, from current levels (Márquez et al., 2014). The shortage of high-quality water supplies has already resulted in the expansion of wastewater treatment and recycling systems to ensure that treated water is healthy for reuse (Kıdak and Doğan, 2018). Over the last decade, the concentration of organic pollutants such as drugs and pesticides has considerably increased, from nanograms to micrograms per liter in urban wastewater, freshwater, and drinking. Contaminants often originate primarily from municipal, hospital, veterinary, and farm wastewater. The growing water pollution is thus of great concern for human health (Kidak and Doğan, 2018; Márquez et al., 2014; Nikolaou et al., 2007).

According to the World Health Organization (WHO) report at 2012, the concentrations of pharmaceuticals in surface water, groundwater, and partially treated water are typically less than $0.1 \mu \mathrm{g} \mathrm{L}^{-1}$, and concentrations in the treated water are generally below $0.05 \mu \mathrm{g} \mathrm{L}^{-1}$ (Organization, 2012). The widespread exposure to medications and pesticides has raised concerns about their adverse effects on the environment and human health. Residues reenter humans and animals' bodies from various water resources and cause adverse effects such as drug resistance and metabolism disruption in the body (Zhang, 2003). Therefore, removing pharmaceuticals from wastewater resources is a pressing issue to safeguard the environment.

Traces of drugs and pesticides in various water resources indicate the low efficiency of traditional systems of water treatment such as filtration, reverse osmosis, and direct contact membrane distillation. Moreover, some pollutants such as diclofenac, paraben, carbamazepine, and diazepam are not fully biologically removable by fungi, bacteria and activated sludge (Dehaghi et al., 2014). Various techniques such as adsorption (Ahmadijokani et al., 2020a, 2020b, 2020c, ndAhmadijokani et al., 2020a, n.d.; Ahmed et al., 2017), reverse osmosis (Rodriguez-Mozaz et al., 2015), air stripping (Zareei and Ghoreyshi, 2011), and biological methods (Ahmed et al., 2017; Carboneras et al., 2018; Goswami et al., 2018; Marican and Duran-Lara, 2018; Saleh et al., 2020) have been deployed widely to remove pollutants, yet adsorption appears promising for several reasons (Ahmad et al., 2010). For instance, adsorption surpass other water treatment strategies because of the lower initial price, higher efficiency, simplicity of layout, and lower maintenance. Furthermore, adsorption does not contribute to the creation of harmful compounds (Ahmad et al., 2010).

Enhanced adsorption can be accomplished using metal oxides, which as crystalline materials that possess a metal cation and an oxide anion, e.g. $\mathrm{MgO}, \mathrm{Al}_{2} \mathrm{O}_{3}, \mathrm{Fe}_{2} \mathrm{O}_{3}, \mathrm{Fe}_{3} \mathrm{O}_{4}, \mathrm{CuO}$, and $\mathrm{ZnO}$ (Gupta et al., 2007; Srivastava et al., 2020). Indeed, metal oxide-based adsorbents bear functional groups, e.g., $-\mathrm{OH},-\mathrm{C}=\mathrm{O}$, and $-\mathrm{COOH}$, and active sites, which can adsorb a wide range of contaminants (Batzill, 2011; Mahmoud et al., 2015; Wu et al., 2016; Bagbi et al., 2017; Li et al., 2016). Carbon-based materials as adsorbents are also under active research because these materials have large surface area, high porosity and surface reactivity, and they can be easily functionalized (Y. Wang et al., 2019; Nazal, 2020). Carbonbased materials such as graphene oxide (Umbreen et al., 2018), activated carbon (Alves et al., 2018), carbon nanotubes (Wang et al., 2019a,b,c), quantum carbon dots (Koe et al., 2020), and graphene (Ali et al., 2019b), are considered the most effective adsorbents for the removal of pollutants, e.g., dyes and pesticides.

The high adsorption capacity of carbonaceous materials has been applied to treat organic pollutants such as drugs, dyes, pesticides and odors (I. Ali et al., 2019b; Umbreen et al., 2018) and for chemical transformations (Varma, 2019). Their unusual physical and chemical properties include exceptionally high tensile strength, high young modulus, good chemical and thermal stability, and excellent thermal and electrical conductivity, thus rendering carbonaceous materials ideal candidates for adsorption applications (I. Ali et al., 2019b; Umbreen et al., 2018). Dissolved organic pollutants are removed from water by water-insoluble carbonbased adsorbents. The adsorption capacity of carbon-based adsorbents depends on porosity, chemical properties and adsorbent durability (Rafati et al., 2018; Shaker and Yakout, 2016; Srivastava et al., 2020). Here we review cytotoxicity effects of drugs and pesticides on humans, and then present the use of metal oxide and carbon nanostructure as efficient adsorbents to remove pesticides and drugs from aqueous media.

\section{Cytotoxic effects of drugs and pesticides in water}

The presence of pesticides and drugs is one of the factors affecting the health of aquatic environments. The major sources, environmental pathways, and toxicity of active pharmaceutical ingredients and pesticides are shown in Fig. 1S (Supplementary file), (Seo et al., 2005). These contaminants change the quality of water resources via their transit from the land to adjacent rivers or water bodies. They are then absorbed by aquatic plants and reach human bodies through the food chain. This pollution transfer cycle causes antagonistic or synergistic effects with acute and long-term results (Bianchi et al., 2017; Hartz et al., 2019; Wolfram et al., 2019). Due to the importance of serious problems caused by the excessive use of these materials, the following sections describe the main impacts of drugs and pesticides on human health.

\subsection{Ecotoxicity of drugs and pesticides}

In recent years, the use of drugs and pesticides has annually increased, and their abuse has become more rampant. These conditions cause drugs and pesticides or their metabolites to persist in the environment as they are relatively stable in the environment and are difficult to control using conventional methods.

\subsubsection{Drugs}

To date, most studies on the residual effects of drugs on human life are focused on drugs used in large amounts, like non-steroidal anti-inflammatory, $\beta$-blockers, fat regulators, and to some extent, psychiatric drugs (Touraud et al., 2011). These lingering drugs illustrate the toxic effects that cause genetic disorderliness, sickness, and cell death. Unhealthy lifestyle behaviors and curative medicines are factors that negatively affect prenatal development and increase the children's susceptibility to diseases. The toxicological mechanisms of chemicals and lifestyle factors include the generation of reactive oxygen species and oxidative cell injury (AlGubory, 2014; Gray et al., 2013). In eco-toxicological terms, dissolved amounts of the drug can be assessed by human blood plasma analysis as an elementary valuation of the eco-toxicological authority of a substance. Then, these results can be compared with blood plasma levels of mammalian in aquatic creatures. Comparative data may reveal whether chronic exposure is required for evaluation in specific long-term trials or not (Länge and Dietrich, 2002). Following the European Directive EC 93/67/EEC (European Commission, 1993), chemical classification is performed based on their EC50 values in three categories; EC50 $<1 \mathrm{mg} \mathrm{L}^{-1}, 1-10 \mathrm{mg} \mathrm{L}^{-1}$, and $10-100 \mathrm{mg} \mathrm{L}^{-1}$ are considered very toxic, toxic, and harmful, respectively, to aquatic organisms (Grabarczyk et al., 2020).

Environmental drug contamination is also found in various states, such as sewage sludge in the ground, leaching from landfills, or irrigating farmable land with treated/untreated effluent in the ground (Kümmerer, 2008). The production and formulation of drugs and their use by patients have caused their improper disposal in the aquatic environment. They mainly enter the aquatic milieu in 
low $\left(<\mathrm{ng} \mathrm{L}^{-1}\right)$ or high concentrations $\left(\mathrm{mg} \mathrm{L}^{-1}\right)$ through human excretion, industrial effluents, and recipient streams with remarkable adverse effects on wildlife and human life (aus der Beek et al., 2016; Boxall et al., 2012; Larsson, 2014). The limited data on measured environmental concentration for bromazepam coincide with a confined eco-toxicological profile. The chronic data on fishes, daphnids, and green microalgae were investigated using the ecological structure-activity relationships-class program (ECOSAR), and the values obtained were 174,6458 , and $2995 \mu \mathrm{g} \mathrm{L}^{-1}$, respectively. For eco-toxicological assays of bromazepam, the behavior of the fish Danio rerio was evaluated and demonstrated the reduction of shoal cohesion. Citalopram with a concentration of $4000 \mu \mathrm{g} \mathrm{L}^{-1}$ presented chronic toxicity for Ceriodaphnia dubia and Daphnia similis during an 8-day experiment (Cunha et al., 2019).

To undertake the environmental risk assessment, the drug concentration in the natural world and the lowest drug concentration with side effects are often examined. Prediction of effets of chemicals parameter on flora and fauna can be ascertained by quantitative structure-activity relationships or common ecotoxicity experiments (Johnson et al., 2008). Consequences for the children include cranial and facial abnormalities and digitalis, along with growth retardation. The required experiments for conducting a robust environmental hazard evaluation of drugs are established by subjecting fishes to genotoxic xenobiotics; effects can increase cancer occurrence, DNA damage, and nuclear abnormalities (Johnson et al., 2008; Priyan et al., 2020).

Anti-cancer drugs mostly induce cytotoxic/genotoxic effects and may damage DNA synthesis and alter the mechanism of endocrine glands regulating tumor growth. Hence, they are considered a threat. There are currently no specific guidelines on how to assess the residual drug risk for human health. However, to evaluate the risk of indirect exposure to drugs, data on the toxicity of drug components are applied to calculate the acceptable daily intake. These acceptable daily intake values are thought to have no pharmacological or toxicological predictive figures but are applied for the predicted no-effect concentrations of human/environmental vulnerability. The predicted no-effect concentrations values can be compared with the predicted environmental concentrations using evaluation patterns (Touraud et al., 2011). The risk ratio of predicted environmental concentrations to predicted no-effect concentrations for human health is presented in Table 1S (Supplementary file), which includes the therapeutic and generic names of some drugs and the acceptable daily intake for drinkable water or fisheating in humans, especially children whose acceptable daily intake are lower than adults. Antibiotics for bacteria control in humans and animals can potentially be hazardous when entering the terrestrial environment. Antibiotic-rich fertilizers used as a source of nutrients for plants raise concerns about the effects of antibiotics on plant growth, soil enzyme/fauna activities, and nutrient cycles. These antibiotics can directly affect the animals and plants in the soil via the ensuing toxicity or indirectly affect the availability of nutrients by altering the microfauna and microflora (Kumar et al., 2005).

One of the discrete pharmacokinetic processes is drug metabolism. In biological drug transfer, lipophilic centers convert to hydrophilic centers to help assist in expelling drugs from the body. Biotransmission is the metabolic degradation of xenobiotics through a specific enzyme process, e.g., the CYP450 family. This process is vital because the drugs with a lipophilic class stay longer in the body. The prolonged shelf life of the drug may lead to poisoning. The well-known pathways of drug metabolism are shown in Fig. 2S (Supplementary file) (De Groot, 2006; Jaladanki et al., 2020).

CYP450 manages the metabolic reactions of drugs, wherein the reactions impact some special atoms of the molecules depending on the electronic factors. Of note, CYP450 controls the entry/exit channel and the catalytic site. The catalytic region of cytochromes comprises three sections of the base, wall, and dome. The drug situations inside the cavity are principally defined based on the chemical characteristics of the dome section (Fig. 3a-S) (Supplementary file); the cavity walls of CYP450 are hydrophobic. The related walls conduct the hydrophobic functions of drugs toward the cavity of Fe-porphyrin. Moreover, this location of drugs is assisted by the amino acids existing in the arch section. Generally, the CYP450 enzyme participates in a catalytic cycle of eight stages (Fig. 3b-S) (Supplementary file):

- Stage 1: replacing the CYP450 enzyme with a water molecule for binding to the substrate.

- Stage 2: reducing Fe(III) to Fe(II) to provide an electron by redox proteins (NADPH-CYP450 reductase).

- Stage 3: binding of $\mathrm{O}_{2}$ with $\mathrm{Fe}(\mathrm{II})$ to electron transfer from $\mathrm{Fe}(\mathrm{II})$ to $\mathrm{O}_{2}$.

- Stage 4-5: enzyme-substrate complexation for taking another electron from redox proteins (cytochrome $b 5$ ).

- Stage 6-7: addition of $\mathrm{H}^{+}$to complex. This complex leads to the failure of $\mathrm{O}-\mathrm{O}$ bond and escape of $\mathrm{H}_{2} \mathrm{O}$ to create Cpd I. Subsequently, $\mathrm{Cpd}$ I withdrew $\mathrm{H}^{+}$from the substrate to set up a radical substrate.

- Stage 8: The oxygen and hydrogen are transferred to the radical substrate and finally release heme iron as $\mathrm{Fe}(\mathrm{III})$. The general catalytic cycle is shown in (Fig. $3 b-S$ ) (Supplementary file):

\subsubsection{Pesticides}

Pesticides are biologically converted to more water-soluble metabolites upon absorption by the body. The biological transfer rate can be fast for organophosphate insecticides (hours to days), or very gradual for organochlorine insecticides, from decades to years. The purpose of biotransformation is essentially detoxification and elimination. The latter event caused the bio-agglomeration of these lipophilic compounds in fatty contexture. Several mechanisms, such as oxidative stress and receptor-mediated mechanisms, are presumed to induce carcinogenicity by pesticides. For oxidative stress, the cytochrome P450 (CYP) catalyzes the normal oxidation of pesticides and produce hydroxylated metabolites (Mechanism 1). CYP450s lead to the oxidative metabolism of organochlorines and polychlorinated biphenyls; hence, the generation of superoxide (Mechanism 2). Other mechanisms including inflammatory and aberrant epigenetic, are in the development stage of the study. Epigenetic rectifications of tumor suppressor genes and their oncogenes altering their tumor expression can also be introduced as molecular stimuli of pathogenesis during cancer progression and progression stages (Banerjee et al., 2001). To evaluate the toxicity and operational mechanisms of pesticide rotenone, tebufenpyrad, and fenazaquin, dose-dependent cell death was studied in the period of exposure 24-48 h. Exposure of cells with various doses of pesticides showed a decrease in ATP and superoxide generation. In other mechanisms, redox-active pesticide metabolites, viz. quinones, or bipyridinium compounds, generate superoxide. Moreover, the electron transport cascades in mitochondria were impaired, producing an excess of superoxide flux. Nicotinamide adenine dinucleotide phosphate (NADPH) oxidase can be activated by pesticides that liberate superoxides (Banerjee et al., 2001; Green et al., 2008; Sherer et al., 2007). The oxidative equations pertaining to CYP and its inefficacious cycling for superoxide production are depicted in Fig. 4S (Supplementary file).

Pesticides may boost the incidence of Parkinson's disease through disruption of mitochondrial function (Sherer et al., 2007). 1-methyl-4-phenyl-1,2,3,6-tetrahydropyridine induces Parkinson's 
disease) with symptoms such as dopaminergic degeneration, $\alpha$ synuclein aggregation, high oxidative damage, and a-synuclein aggregation by inhibition of complex I. Dose-dependent cell death of human neuroblastoma cell line (SK-N-MC) neuroblastoma cells, exposed to pesticide treatment, exhibited a rank order toxicity: pyridaben $>$ rotenone $>$ fenpyroximate $>$ fenazaquin $>$ tebufen pyrad. The most toxic compound of pyridaben illustrated the significant toxicity at a concentration of $10 \mathrm{pmol} \mathrm{L}^{-1}$ (picomoles per liter), while the toxicity of tebufenpyrad and fenazaquin was attained at a concentration of $1 \mathrm{M}$ (Sherer et al., 2007). Also, pesticides can cause stinging eyes, rashes, blisters, blindness, nausea, dizziness, diarrhea and death. Examples of known chronic effects are cancers, birth defects, reproductive harm, neurological and developmental toxicity, immunotoxicity, and disruption of the endocrine system (Hallenbeck and Cunningham-Burns, 2012).

Although pesticides are not genotoxic by themselves, their binding efficiency to steroid and xenobiotic receptors may lead to creating alterations in gene expression programs. Pesticideinduced cancers appear to be caused by effects on biomarkers, genetic sensitivity, oxidative stress, and DNA lesion (Gray et al., 2013). Based on the available epidemiological evidence, there is a link between pesticide exposure and cancer. Alteration by pesticides may damage DNA and contribute to cancer mutagenic mechanisms. Many xenochemicals can block or mimic the hormone function by attaching the endogenous ligands to the steroid nuclear receptor family and displacing them, thereby activating the function of the receptor, leading to a change in gene expression (Kretschmer and Baldwin, 2005; Schug et al., 2011). Moreover, insecticides can stress midgut cells, and heat shock protein 70 (HSP70) can eliminate this damage. Major damage of morphological alterations was precluded through the amplified expression of this stress protein. The localization of HSP70 may be related to areas where detoxification is performed (de Souza et al., 2019). Leading pesticide compounds and their action mechanism on body organisms are summarized in Table $2 \mathrm{~S}$ (Supplementary file).

There is evidence that persistent organic pollutants are related to type 2 diabetes. Inflammation in fatty organs, lipotoxicity in living organs such as pancreas, liver, muscle, and mitochondrial abnormality or impairment leads to the obesity and type 2 diabetes epidemic's initial mechanism. Persistent organic pollutants contaminate the food materials and our environments; hence, they can increase human's adipose tissue after accumulation in the tissue. Persistent organic pollutants with continuous release into circulation can reach critical organs and induce chemical-specific or physiological responses. The low doses of persistent organic pollutants induce three phases (viz., phase I, phase II, and phase III xenobiotic metabolism pathways) that enhance the excretion of persistent organic pollutants. Phase II leads to chronically decreasing inconsumption of mitochondrial function. This decrease in glutathione wreck the mitochondrial function and the reduction is acutely dependent on the inflammation and lipid accumulation of extrauterine. On the other hand, an increased amount of persistent organic pollutants mixtures can enhance proteins' synthesis with cytoprotective and restorative functions. This activation may ameliorate mitochondrial function and theoretically reduce the risk of type 2 diabetes. Therefore, U-shaped inverted associations can be expected. In other words, persistent organic pollutants can induce a dual effect on adipose (i.e., alteration in hyperplasia and hypertrophy) by various endocrine disrupting mechanisms depending on the type or dose of POPs (Han et al., 2020; Lee et al., 2014; Mansouri and Reggabi, 2020). Fig. 5S (Supplementary file) presents the overview of correlations of persistent organic pollutants and type 2 diabetes and their conceivable mechanisms.

\section{Environmental and human risks of metal oxides and carbon-based nanoparticles}

The success of nanotechnology is due to the unique properties of nanoparticles. Although nanoparticles have numerous advantages in various arena of the scientific world and in the daily life of human communities (Makvandi et al., 2020; C.Y. Wang et al., 2020), they may also have toxic effects on the plant, animals, and humans. A large quantity of nanoparticles has recently entered the environment because of the growing applications and development. Humans and animals are exposed to the nanoparticles through inhalation, swallow, and skin uptake, and after nanoparticles entry, they can damage the cells and ultimately the organs by complicated mechanisms (Sengul and Asmatulu, 2020). However, toxicity of metal oxide nanoparticles and carbon-based nanomaterials is not well known and is still being assayed for potential ecological and human health hazards. According to the obtained results from invivo and in-vitro assays, the cytotoxicity and gene toxicity of metal oxide nanoparticles on animals is related to the production of reactive oxygen species (ROS) and oxidative stress that can lead to lipid peroxidation, inflammation induction, animals' organs damage, the nervous system interference, DNA damage, and affect offspring (Srivastava et al., 2020; Zhu et al., 2019). There are few reports on the toxicity of metal oxide nanoparticles to human's body (Djurišić et al., 2015; Schneider et al., 2017), especially the influences of metal oxide nanoparticles on oral cells, mucosal cells, so these toxic influences on human life should not be ignored, and more study in this field is required in the future.

The industrial usage and widespread application of carbonbased nanomaterials have caused a rapid increase in their production, so the anxiety about their harmful effects on the environment and human is raised. Carbon-based nanomaterials have possible long-term ecological consequences, while the ecological and human health dangers of these nanomaterials have not been systematically investigated. Cytotoxicity towards animal, algae, and bacteria cells has been observed for graphene oxide, reduced graphene oxide (Shareena et al., 2018), graphene quantum dots (Hashemi et al., 2020), and carbon nanotubes chiefly multi-walled carbon nanotube.

The graphene oxide cytotoxic effect to microbial communities was confirmed at $50-300 \mathrm{mg} \mathrm{L}^{-1}$, and there was no substantial decrease in viability of human cells after exposure to the noncytotoxic dosage of $15 \mu \mathrm{g} \mathrm{mL} \mathrm{m}^{-1}$ for graphene oxide $(100 \mathrm{~nm})$ and graphene quantum dots $(50 \mathrm{~nm})$ (Hashemi et al., 2020). The considerable alterations in the expression level of Bax, Bcl2, miR-21, miR-29a, and PTEN genes, and changes in mitochondrial activity at cellular level were observed after treatment with non-cytotoxic dosage. The basal level of genes and mitochondrial membrane potential were more influenced by the graphene oxide in compared to graphene quantum dots. The miRNA alteration can alter the fate of cells, and all these genes are involved in the development and metastasis of breast tumor. Thus, the secondary toxic influence of graphene oxide-100 and graphene quantum dots-50 at a non-toxic dosage was confirmed on both, the cellular and molecular level.

The carcinogenic risks of carbon nanotubes were published by the International Agency for Research on Cancer (IARC) in 2014 (Kobayashi et al., 2017). The carcinogenicity effect of single wall carbon nanotube is less than multi wall carbon nanotube for humans (Heller et al., 2020), and the suspected carcinogenic effects of single wall carbon nanotube ( $>55 \%$; diameter $<2 \mathrm{~nm}$ and length $=5-15 \mu \mathrm{m}$ ) was reported by both IARC and chemical secretariat monographs. Exposure to multi wall carbon nanotubes stimulate local and systemic inflammation, contributing to formation of the pre-metastatic and metastatic niches, along with 
damaging the central nervous system (Falahian et al., 2018) and promoting the metastatic cascade of breast cancer (Lu et al., 2019). The detrimental health influences in animal studies comprise cardiac inflammation, acute and chronic respiratory damage, and cancer such as mesothelioma (Ellenbecker et al., 2018). The longterm sustainability of carbon nanotubes for water treatment is questionable without the nano-safety guidelines, and so the current risk measurements of carbon nanotubes are overshadowed by uncertainties (Das et al., 2018).

Despite the intrinsic cytotoxicity of metal oxides and carbonbased nanomaterials as described above, abundant outstanding features of these nano-adsorbents make their application in the water treatment field extremely attractive. As a result, the topic need to address how to make the useful appliance of nanoparticles in terms of benefits while decreasing their toxic impact on the environment and human life.

\section{Adsorption process and the adsorbents}

The term adsorption, initially introduced by Kayser in 1881 (Dąbrowski, 2001), is a physicochemical process that typically happens in the solid-liquid form. Although adsorption is fully efficient in both liquid-liquid and gas-liquid phases, it is primarily used to treat water in solid-liquid phases. Adsorption is the mechanism by which molecules of a liquid or gaseous material are bound to a solid adsorbent (Dąbrowski, 2001; del Mar Orta et al., 2019; Derylo-Marczewska et al., 2017) and this process requires the aggregation of materials at the interface between the two phases. Essentially, the adsorption of molecules by internal or exterior surfaces of a solid could be termed as adsorption. The substance taken out of the liquid phase is called the adsorbate, and the material providing the adsorption surface is called the adsorbent. The adsorption process can be classified into three groups: physisorption, chemisorption, and ion exchange. Van der Waals and covalent forces are the main forces that control physical and chemical adsorption, respectively (related to the bonding between the adsorbent and the adsorbent) (Crini et al., 2018). Several factors like the $\mathrm{pH}$ of the aqueous solution, the sorbent dosage, the contact time, the concentration of the main substances, and the temperature hugely influence the adsorption of pollutants. The optimization of these factors plays a significant role in the adsorption of contaminants (Ali et al., 2019a), which is invariably accompanied by the release of energy. The heat released from the physical and chemical adsorption is between 5-10 and 10-100 kcal mol ${ }^{-1}$, respectively (Ali et al., 2019a; Crini et al., 2018).

Adsorbents play an important role in the elimination of water contaminants and their efficiency depends on the structural features. The important properties of good adsorbent comprise biocompatibility, non-toxicity, readily available, simple renewability, and biodegradability (Nasrollahzadeh et al., 2021a). They must have great adsorption capacity due to the high surface area, along with good thermal stability and high abrasion resistance. Adsorbent compounds can be organic, mineral, or biological, and they are usually employed in the form of spherical, rod, wire, tube, or particle with sizes ranging from nanometer to millimeter (Singh et al., 2018). The adsorbents are generally categorized into three classes: (I) polar and hydrophilic oxygen-containing materials such as metal oxides (Gusain et al., 2019), (II) non-polar and hydrophobic carbon-based materials such as activated carbon, graphite, graphitic carbon nitride, graphene, carbon nanotubes, carbon nanofibers, fullerenes, and nanodiamonds (Nasrollahzadeh et al., 2021b), and (III) polar or non-polar polymer-based materials (Ismail et al., 2020). A great deal of effort has been expanded over the last few years on the usage of various adsorbents for water/ wastewater purification (Srivastava et al., 2020; Zare et al., 2018).

\subsection{Metal oxides}

Metal oxide nanoparticles exhibit significant potential for wastewater remediation due to their low toxicity, thermal stability, large surface area, porous structures, easy recovery, and the presence of Lewis acid-base sites in their structures (Nagpal and Kakkar, 2019).

\subsubsection{Iron oxides}

Iron oxide nanoparticles, in the pure, doped, and composite forms, have been widely used for the adsorption of organic pollutants (D'Cruz et al., 2020; Shrivas et al., 2019). Magnetic nanoparticles, including hematite $\left(\alpha-\mathrm{Fe}_{2} \mathrm{O}_{3}\right)$, maghemite $\left(\gamma-\mathrm{Fe}_{2} \mathrm{O}_{3}\right)$, and magnetite $\left(\mathrm{Fe}_{3} \mathrm{O}_{4}\right)$, were commonly deployed for the elimination of contaminants from aqueous media (Bhateria and Singh, 2019). The reason for the popularity of magnetic nanoparticles as an adsorbent is their easy separation via an external magnetic field and the reduced energy consumption and costs in the recycling process; the use of magnetic nanoparticles in the adsorption of organic pollutants has been highlighted (Bhateria and Singh, 2019). For example, superparamagnetic iron oxide nanoparticles (SPIONP, $\mathrm{Fe}_{3} \mathrm{O}_{4}$ ) have been synthesized via the co-precipitation procedure and used for the sorption of levofloxacin (LEV) drug from aqueous solutions (AlJabari et al., 2019). The maximum removal percentage of levofloxacin by superparamagnetic $\mathrm{Fe}_{3} \mathrm{O}_{4}$ was achieved at $\mathrm{pH} \sim 6.5$, which indicated that the affinity of neutral/zwitterion form of levofloxacin onto the adsorbent is greater than their cationic and anionic counterparts. Based on these data, a plausible mechanism was proposed, which is schematically depicted in Fig. 1.

Modified iron oxide nanoparticles have good potential for the wastewater treatment process as has been exemplified in the rapid green synthesis of magnetic nano-sorbents by using extracts such as Nelumbo nucifera stalks, Colocasia esculenta corms, and Piper betle leaves; they provide an efficient, facile, and environmentally friendly route for the modification of nanoparticles (Misra et al., 2018), and as illustrated, in the good adsorption efficiency for carbamazepine.

Also, the green synthesized iron oxide nanoparticles (IONPs; $\mathrm{Fe}_{3} \mathrm{O}_{4}$ ) using an extract of Excoecaria cochinchinensis leaves (Lin et al., 2018) were applied for the elimination of rifampicin (RIF) from aqueous media (Fig. 2) (Cai et al., 2019); isotherm and kinetic results demonstrated the monolayer chemisorption of rifampicin onto IONPs with a $\mathrm{q}_{\max }$ of $84.8 \mathrm{mg} \mathrm{g}^{-1}$. The adsorption mechanism of rifampicin onto $\mathrm{Fe}_{3} \mathrm{O}_{4}$ nanoparticles at optimum $\mathrm{pH} 5.5$ comprised electrostatic attraction and chemisorption between active sites of $\mathrm{Fe}_{3} \mathrm{O}_{4}(-\mathrm{COOH},-\mathrm{COH}$, and $\mathrm{C} \pi$ electron) with functional groups of the rifampicin molecules.

A greener protocol for the preparation of crystalline hexagonal hematite nanoparticles has been reported using the supernatant culture of a newly isolated bacterium, Bacillus cereus SVK1 (Rajendran et al., 2015). The applicability of eco-friendly bio-synthesized hematite nanoparticles was explored for the adsorptive removal of carbamazepine from water (Rajendran and Sen, 2018). The main reason for high removal percent $(>90 \%)$ is believed to be related to the hydrogen bonding between amide groups of carbamazepine and the functional groups on hematite.

Modified nanoparticles with functional groups increase the surface functionality along with the surface wettability and stability (Gao and Yan, 2012). Indeed, the hydrophobic, electrostatic, van der Waals, $\pi-\pi$ interactions and hydrogen bonding of adsorbent functional groups cause the adsorption of organic contaminants onto the modified metal oxides. For example, the magnetic sorbent (103-HC-DS/Fe-5) was synthesized via modification of hydrocalumite-iron oxide with dodecyl sulfate to remove agrichemicals, atrazine $\left(\mathrm{q}_{\max }=4.5 \mathrm{mg} \mathrm{\textrm {g } ^ { - 1 }}\right)$ and chlorpyrifos 


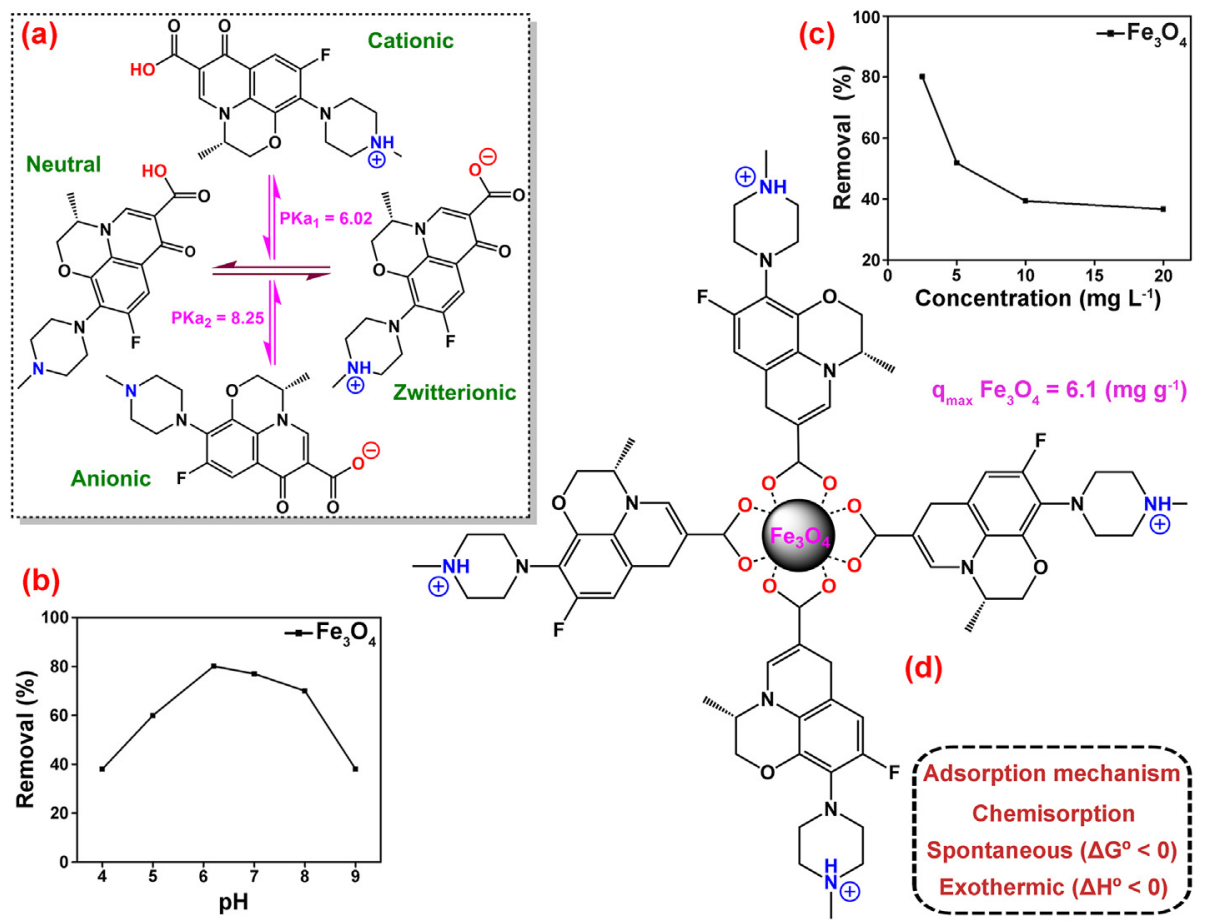

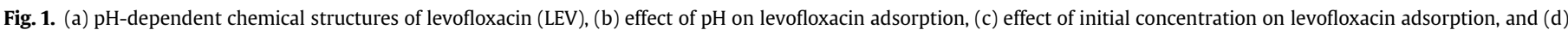

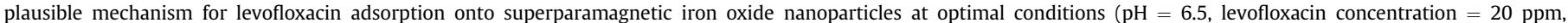
time $=240 \mathrm{~min}$, and temperature $=298 \mathrm{~K}$ ). Reproduced with modification with permission from (Al-Jabari et al., (2019)).

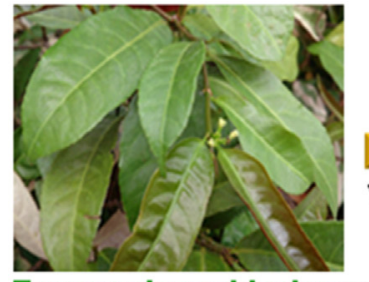

Excoecaria cochinchnensis

(EC)

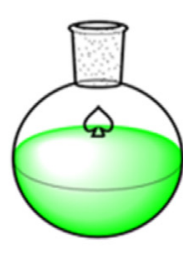

EC Extract
$\mathrm{CH}_{3}$ COONa

$\mathrm{FeCl}_{3} \cdot 6 \mathrm{H}_{2} \mathrm{O}$

$\left(70^{\circ} \mathrm{C}, 2 \mathrm{~h}\right)$

Heat $\left(80^{\circ} \mathrm{C}, 1 \mathrm{~h}\right)$

Vacuum-filtration

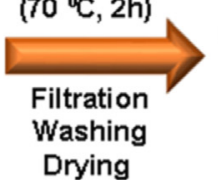

Drying
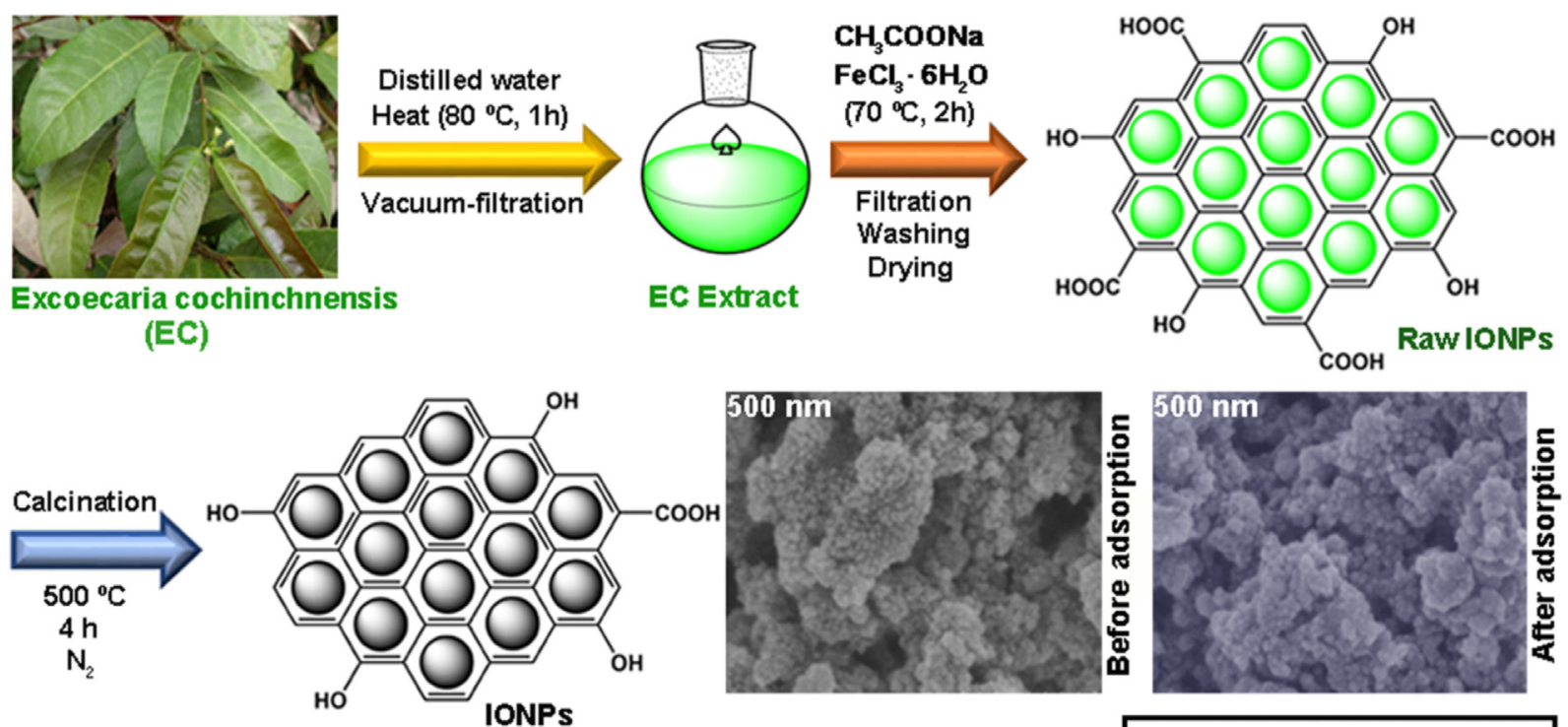

是 $\left(\mathrm{S}_{\mathrm{BET}}=111.8 \mathrm{~m}^{2} \mathrm{~g}^{1}\right)$

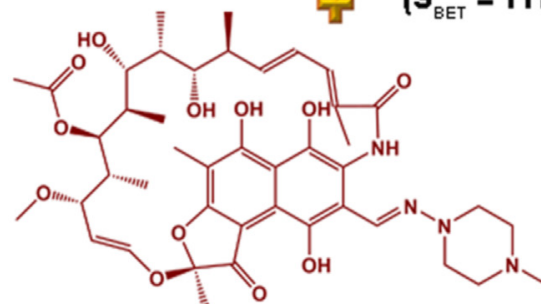

Rifampicin (RIF): pKa1 = 1.7, pKa2 = 7.9

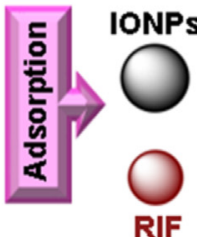

RIF

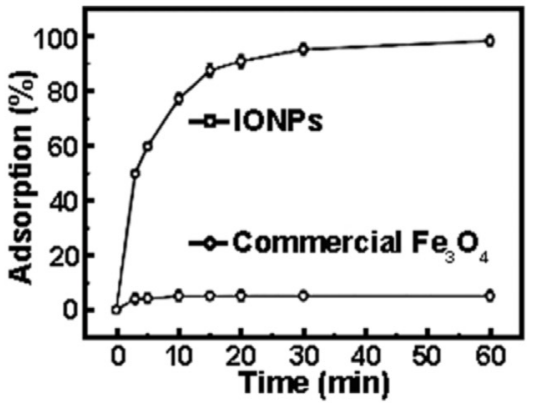

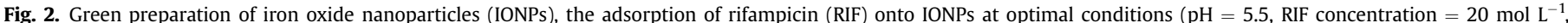

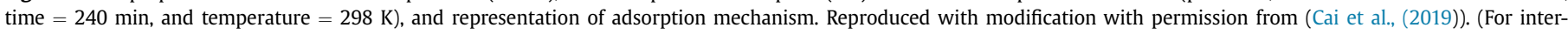
pretation of the references to colour in this figure legend, the reader is referred to the Web version of this article.) 
$\left(\mathrm{q}_{\max }=72.9 \mathrm{mg} \mathrm{g}^{-1}\right)$, from aqueous solutions (Milagres et al., 2020). Further, Milagres et al. reported that the 103-HC-DS/Fe-5 sorbent could be successfully utilized for the elimination of acetamiprid, atrazine, chlorpyrifos, and thiamethoxam agrichemicals from river water (Milagres et al., 2020).

The $\mathrm{Fe}_{3} \mathrm{O}_{4}$-red mud nanoparticles have been prepared and used for the removal of ciprofloxacin from wastewater via a batch technique (Aydin et al., 2019); their adsorption capacity for ciprofloxacin was determined to be $111.11 \mathrm{mg} \mathrm{g}^{-1}$ and the removal percent for ciprofloxacin being over $90 \%$ from real wastewater. The combination of $\mathrm{Fe}_{3} \mathrm{O}_{4}$ and red mud has overcome the disadvantages such as iron leaching, agglomeration of nanoparticles, and separation difficulties from aqueous solutions relating to pristine materials. The low price and ready availability of the red mud as processes waste renders the $\mathrm{Fe}_{3} \mathrm{O}_{4}$-red mud nanoparticles a suitable alternative to commercial adsorbents.

Facile and one-pot hydrothermal procedure have been documented for the preparation of pure hematite $\left(\alpha-\mathrm{Fe}_{2} \mathrm{O}_{3}\right)$ nanoparticles (Nassar et al., 2018) with high adsorption capacity (70 $\mathrm{mg} \mathrm{g}^{-1}$ ) for the elimination of cephalexin antibiotic from aqueous media at optimum conditions $\left(\alpha-\mathrm{Fe}_{2} \mathrm{O}_{3}\right.$ dosage $=0.05 \mathrm{~g}$, cephalexin solution volume $=25 \mathrm{~mL}, \mathrm{pH}=7.5$, cephalexin initial concentration $=150 \mathrm{mg} \mathrm{L} \mathrm{L}^{-1}$, time $=180 \mathrm{~min}$, and temperature $=25^{\circ} \mathrm{C}$ ). The strong electrostatic attractions between the $-\mathrm{CO}_{2}^{-}$groups of negatively charged deprotonated-cephalexin molecules $\left(\mathrm{pKa}_{2}<7.5\right)$ and the positively charged $\alpha-\mathrm{Fe}_{2} \mathrm{O}_{3}$ nanoparticles ( $\mathrm{pH}$ PZC of $\alpha-\mathrm{Fe}_{2} \mathrm{O}_{3}>7.5$ ) were responsible for the obtained maximum adsorption capacity at $\mathrm{pH}=7.5$.

Some studies have been reported on the adsorption of oxolinic acid, levofloxacin, diclofenac, ketoprofen, and naproxen by goethite adsorbent, and have been demonstrated the good adsorption efficiency of geothite (Cheng et al., 2019; Qin et al., 2018; Yu et al., 2019). Mechanistic insight into interactions between two iron oxide minerals (IOMs; ferrihydrite and goethite) with different crystal structures and tetracycline was presented by Wu et al. (Fig. 3) (Wu et al., 2019). It was indicated that ferrihydrite, an amorphous iron oxide with a larger specific surface area and smaller pore size, exhibited a better adsorption capability than goethite, a crystalline iron oxide, to remove of tetracycline from aqueous media; both IOMs formed stable inner-sphere complexation and had specific tetracycline sorption. Although both IOMs systems had electrostatic interactions with tetracycline sorbate, the significant effect for tetracycline adsorption onto goethite was only discernible. After tetracycline adsorption on ferrihydrite, $\mathrm{Fe}^{2+}$ ion was released in solution, indicating a redox chemical reaction. $\mathrm{Na}^{+}$and $\mathrm{Ca}^{2+}$ ions had no significant influence on tetracycline sorption, whereas $\mathrm{Cu}^{2+}$ ions formed a complex with tetracycline and its size became larger. Therefore, the $\mathrm{Cu}$ tetracycline complex reduced the tetracycline sorption onto ferrihydrite with a smaller pore size but improved tetracycline sorption onto goethite. The ensuing results from the ionic force influence provided proof about iron oxide mineral effect on the mobility of tetracycline.

An innovative approach for the adsorption and persulfate-based advanced oxidation of ibuprofen has been reported by Yin et al. using recycled rusted iron particles (RRIP) from heat pads (Fig. 4) (Yin et al., 2018); RRIP core-shell structure comprises zero-valent iron $\left(\mathrm{Fe}^{0}\right)$ in the core, and amorphous $\mathrm{FeOOH}$ and $\alpha$-FeOOH on the shell. The $\mathrm{q}_{\max }$ of RRIP for ibuprofen sorption was $3.47 \mathrm{mg} \mathrm{g}^{-1}$, which was 4.8 times greater than the synthetic $\alpha$-FeOOH. The larger pore volume/size and higher specific surface area of RRIP compared with $\alpha$-FeOOH was the reason for the enhanced ibuprofen adsorption. Also, the iron (hydr)oxides created on the rusted iron particles surface generate a large number of sites for the adsorption of ibuprofen. The RRIP activated persulfate can degrade the adsorbed and dissolved ibuprofen by generating both the hydroxyl $(\mathrm{OH} \cdot)$ and sulfate $\left(\mathrm{SO}_{4}^{-} \cdot\right)$ radicals.

\subsubsection{Zinc oxide}

Other metal oxides or their composites have been investigated either theoretically or experimentally for the removal of drug and (a)
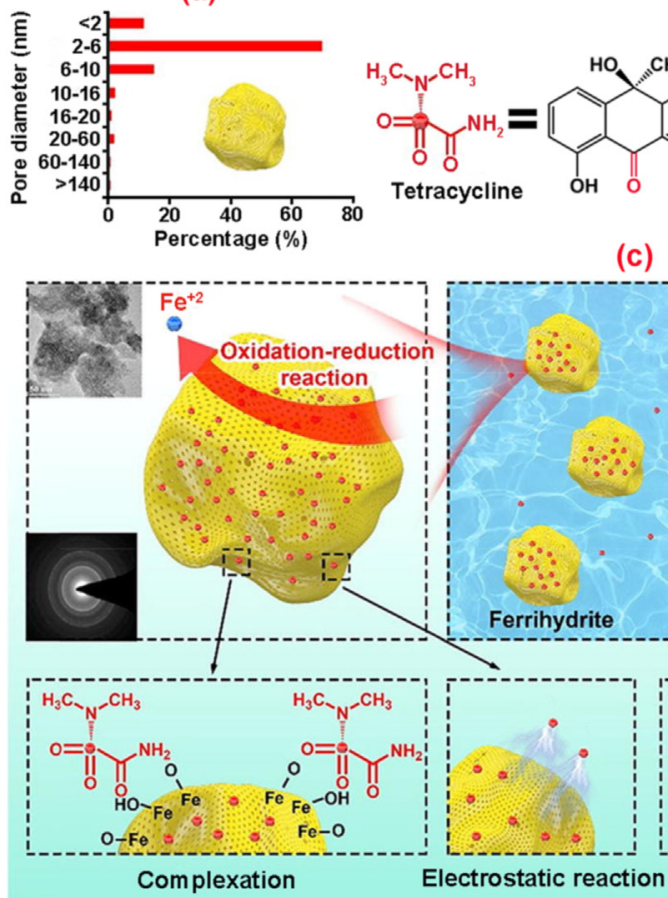

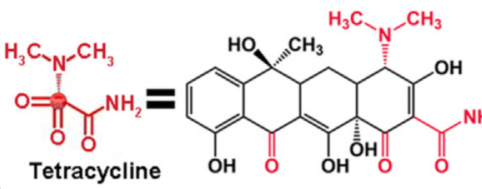

(c)

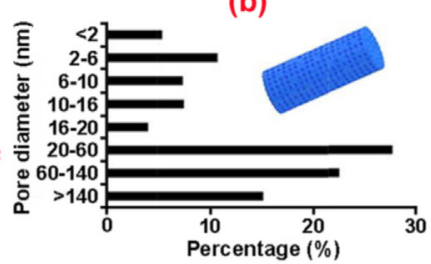

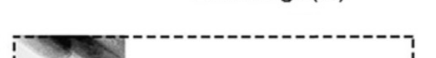

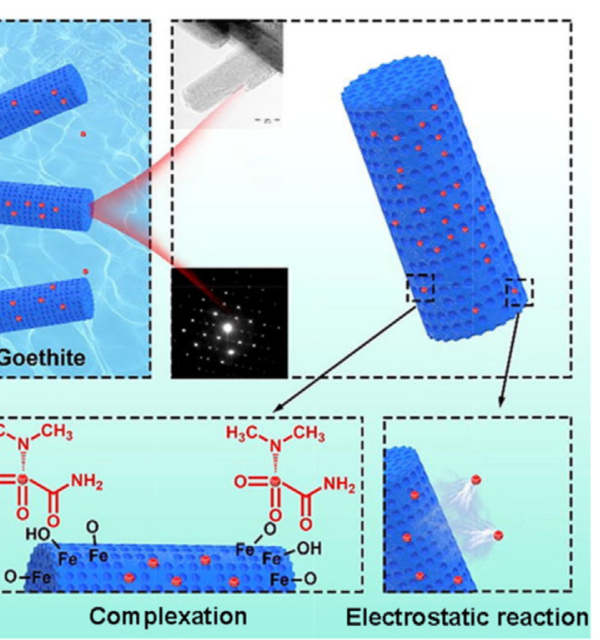

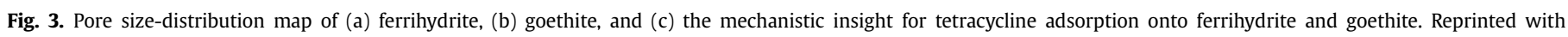
permission from (Wu et al., (2019)). 


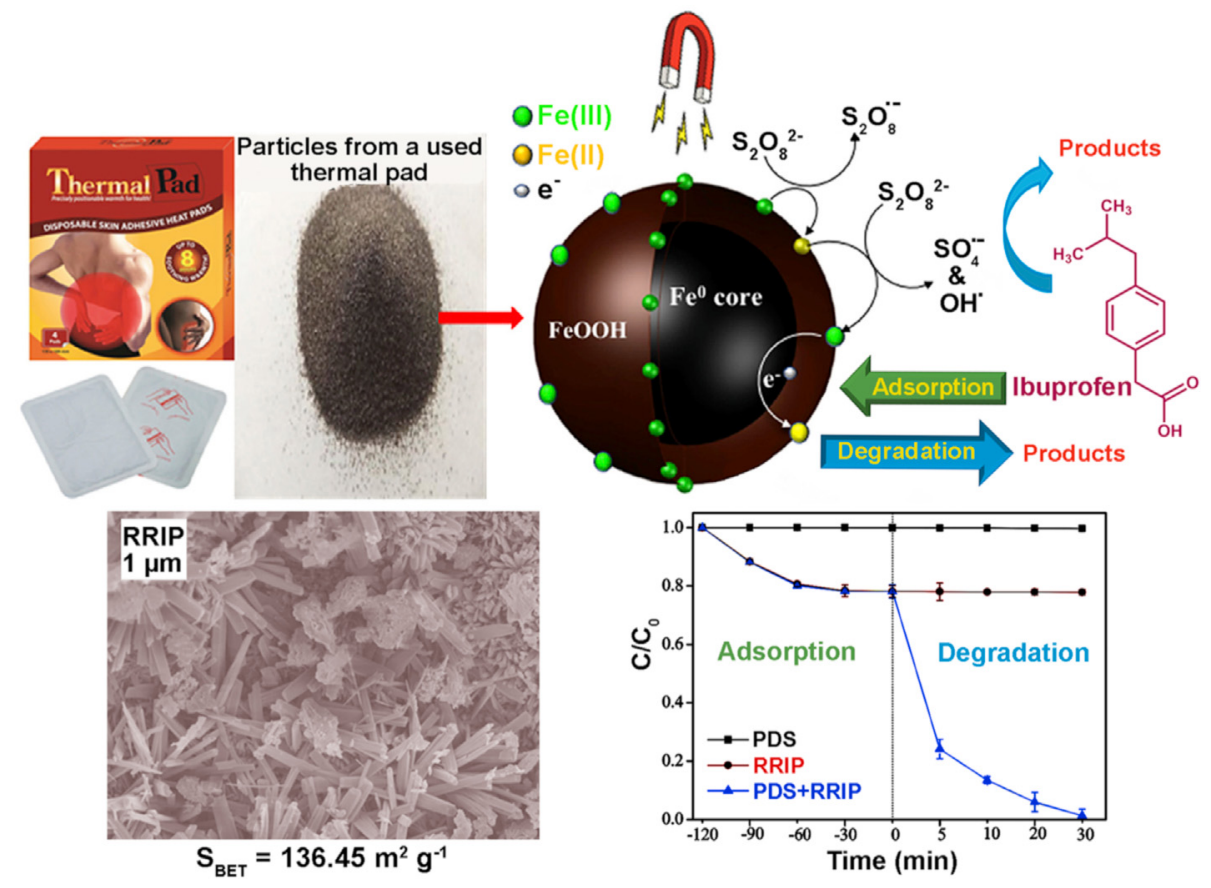

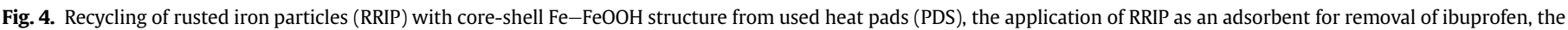

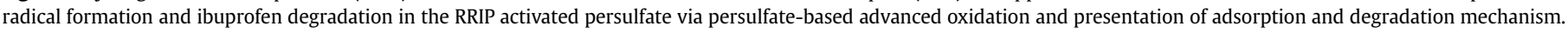
Reprinted with modification with permission from (Yin et al., (2018)).

pesticide residues. The $\mathrm{Zn}_{12} \mathrm{O}_{12}$ nanocluster containing 6 tetragonal and 8 hexagonal rings with $36 \mathrm{Zn}-\mathrm{O}$ bonds was synthesized by Onsori et al., and its adsorption capability and electronic sensitivity to the anticancer drug platinol, was explored via density functional theory (DFT) calculations in aqueous solution (Onsori and Alipour, 2018). Platinol simultaneously interacted with $\mathrm{Zn}$ and $\mathrm{O}$ atoms of $\mathrm{Zn}-\mathrm{O}$ bond via the $\mathrm{Cl}, \mathrm{Pt}$, and $\mathrm{H}$ atoms and lay on a $\mathrm{ZnO}$ nanocluster hexagonal ring with an adsorption energy of $-31.7 \mathrm{kcal} \mathrm{mol}^{-1}$ $(298 \mathrm{~K})$. After drug adsorption, the $\mathrm{ZnO}$ nanocluster valence level shifted to higher energies, and the high destabilization of the HOMO level decreased the HOMO-LUMO energy gap (Eg) of the $\mathrm{ZnO}$ nanocluster. Thus, Eg decrease and conduction electrons increase of adsorbent largely enhanced the electrical conductivity. The enhancement in $\mathrm{ZnO}$ nanocluster electrical conductivity after platinol adsorption was converted to an electrical signal, thus rendering $\mathrm{ZnO}$ nanocluster a promising candidate for platinol detection.

Besides theoretical investigations, the $\mathrm{ZnO}$ nanoparticles that were prepared via the precipitation method were evaluated for the elimination of ciprofloxacin hydrochloride from aqueous media via batch experiments (Dhiman and Sharma, 2019a). The highest adsorption of $85.4 \%$ at $\mathrm{pH} 4$ and assessment of isotherm and kinetic equations for ciprofloxacin antibiotic adsorption onto $\mathrm{ZnO}$ nanoparticles illustrated that favorable monolayer sorption with uniform distribution of sorbate onto sorbent surface occurred via physical interactions.

In another report, competitive removal of [ofloxacin hydrochloride + ciprofloxacin hydrochloride] and [paracetamol + diclofenac sodium] from binary solutions was studied using ZnO nanoparticles (Dhiman and Sharma, 2019b). The $\mathrm{ZnO}$ nanoparticles displayed good adsorption efficiency for the studied drugs in primary solutions and $\mathrm{pH}$ played an important role in drug sorption onto $\mathrm{ZnO}$ nanoparticles. The optimal $\mathrm{pH}$ was chosen at $\mathrm{pH}=8$; the $\mathrm{pH}$ below $\mathrm{ZnO}$ point zero charge $\left(\mathrm{pH}_{\mathrm{PZC}}=8.7\right), \mathrm{pKa}_{1}<\mathrm{pH}<\mathrm{pKa}_{2}$ for ofloxacin and ciprofloxacin, $\mathrm{pKa}$ diclofenac sodium $<\mathrm{pH}$, and $\mathrm{pKa}$ paracetamol $>\mathrm{pH}$. Thus, there were strong electrostatic attractions between the positively charged surface of $\mathrm{ZnO}$ with the negatively charged carboxyl group of zwitterionic ofloxacin and ciprofloxacin, as well as with anionic diclofenac but the hydrophobic and electrostatic interactions did not play a role in the paracetamol adsorption. In binary solutions, the presence of the second drug had a negative effect on the removal of the first drug, and vice versa. So, the adsorption of each drug was significantly reduced in the presence of other drugs.

\subsubsection{Cerium oxide}

The preparation of $\mathrm{CeO}_{2}$ nanofibers from $\mathrm{Ce}(1,3,5$-benzene tricarboxylate) $\left(\mathrm{H}_{2} \mathrm{O}\right)_{6}$ (Ce-BTC) metal-organic frameworks and their application was investigated for the pesticide adsorption from aqueous media (Abdelillah Ali Elhussein et al., 2018). The Ce-BTC metal-organic frameworks were synthesized via the hydrothermal method and $\mathrm{CeO}_{2}$ nanofibers were created from Ce-BTC nanoparticles by calcination method at $650{ }^{\circ} \mathrm{C}$ for $3 \mathrm{~h}$. The $\mathrm{CeO}_{2}$ nanofibers were used for 2,4-dichlorophenoxyacetic acid (2,4-D) adsorption from the water by a batch system with the $\mathrm{q}_{\max }$ value to be $95.78 \mathrm{mg} \mathrm{g}^{-1}$ at $308 \mathrm{~K}$. Based on the kinetic and isotherm studies, the 2,4-D sorption onto $\mathrm{CeO}_{2}$ was performed through boundary layer diffusion and intra-particle diffusion.

\subsubsection{Cobalt oxide and copper oxide}

Other research works for drug adsorption onto metal oxides were exemplified by doxycycline hyclate sorption onto cobalt oxide $\left(\mathrm{Co}_{3} \mathrm{O}_{4}\right)$ (Abbas et al., 2019) and ciprofloxacin hydrochloride adsorption onto copper oxide (CuO) (Sharma and Dhiman, 2017) in aqueous solutions.

\subsubsection{Metal oxides composites}

Despite the good performance of metal oxide nanoparticles, metal oxide composites had a better performance for removing organic pollutants due to the synergistic effects of metal oxides 
participating in their structures. Magnetically retrievable mesoporous magnesium ferrite $\left(\mathrm{MgFe}_{2} \mathrm{O}_{4}\right)$ has been explored for chlorpyrifos adsorption from real pesticide wastewater (Sharma and Kakkar, 2018). The mesoporous $\mathrm{MgFe}_{2} \mathrm{O}_{4}$ with high surface area $\left(170 \mathrm{~m}^{2} \mathrm{~g}^{-1}\right)$ was prepared using benign starting materials and urea as a dual-purpose mediator in the one-step solvothermal method (Fig. 5a). Batch adsorption experiments demonstrated that the effective adsorption of chlorpyrifos occurred onto $\mathrm{MgFe}_{2} \mathrm{O}_{4}$ adsorbent at $\mathrm{pH}>9$ (Fig. 5b); the proposed chlorpyrifos adsorption mechanism is depicted in Fig. 5c. Accordingly, a chemisorption process occurred via the interaction of hydroxylated $\mathrm{MgFe}_{2} \mathrm{O}_{4}$ surface with the electronegative atoms (chlorine, oxygen, and sulfur) and the chlorpyrifos aromatic ring followed by degradation into small organic species. This investigation revealed that the mesoporous $\mathrm{MgFe}_{2} \mathrm{O}_{4}$ with high adsorption efficiency can be an interesting selection for wastewater treatment.

The adsorption capability of $\mathrm{ZnO}-\mathrm{MgO}$ nanocomposite and $\mathrm{MgO}$ nanoparticles were evaluated and compared for the elimination of antibiotics from aqueous media via response surface methodology (Fakhri and Behrouz, 2015); fast and efficient linezolid adsorption occurred onto $\mathrm{MgO}$ nanoparticles and $\mathrm{ZnO}-\mathrm{MgO}$ nanocomposite via a simple procedure. The maximum adsorption capacity of $\mathrm{ZnO}-\mathrm{MgO}$ nanocomposite (140.28 $\mathrm{mg} \mathrm{g}^{-1}$ ) was better than $\mathrm{MgO}$ nanoparticles (123.45 $\mathrm{mg} \mathrm{g}^{-1}$ ) because of the higher specific surface area of the nanocomposite.

The composite of iron and copper oxides ( $\mathrm{Fe} / \mathrm{Cu}$ oxides) has been synthesized using the extract of pine needle and was applied as an efficient adsorbent for ofloxacin and norfloxacin removal from aqueous media (Fig. 6a) (Ma et al., 2020). The maximum adsorption capacity of $\mathrm{Fe} / \mathrm{Cu}$ oxide for ofloxacin and norfloxacin were calculated $1.26 \mathrm{mmol} \mathrm{g}^{-1}$ and $1.64 \mathrm{mmol} \mathrm{g}^{-1}$, respectively. The thermodynamic parameters established the endothermic and spontaneous nature of adsorption, and kinetic results revealed that the diffusion had an important role in the adsorption rate-limiting step. The ofloxacin and norfloxacin adsorption mechanism involved a combination of electrostatic attraction $\left(\mathrm{pH}<\mathrm{pH}_{\mathrm{PZC}}<\mathrm{pKa}_{1}\right)$, hydrogen bonds formation, $\pi-\pi$ stacking, hydrophobic interaction, and surface complexation (Fig. 6b).

Similar studies were performed in the field of water purification by metal oxides nanocomposites for the removal of anticonvulsant drug oxcarbazepine onto 3D porous $\mathrm{NiFe}_{2} \mathrm{O}_{4}$ (Parashar et al., 2020) and adsorption of riboflavin onto silver-deposited iron oxide magnetic $\left(\mathrm{Ag} / \mathrm{Fe}_{3} \mathrm{O}_{4}\right)$ nanoparticles (Akhond et al., 2016). The excellent adsorption efficiencies demonstrated by the most used metal oxides and their composites for various drugs/pesticides removal are presented in Table 1.

\subsection{Carbon nanostructures}

Carbon is among the most plentiful and versatile elements on the earth with a wide-ranging application (Benzigar et al., 2018). Carbon-based nano-compounds exhibit special and extraordinary characteristics, including tremendous thermal and mechanical stability, corrosion resistance, hardness, and low density (Z. M. Li

(a)

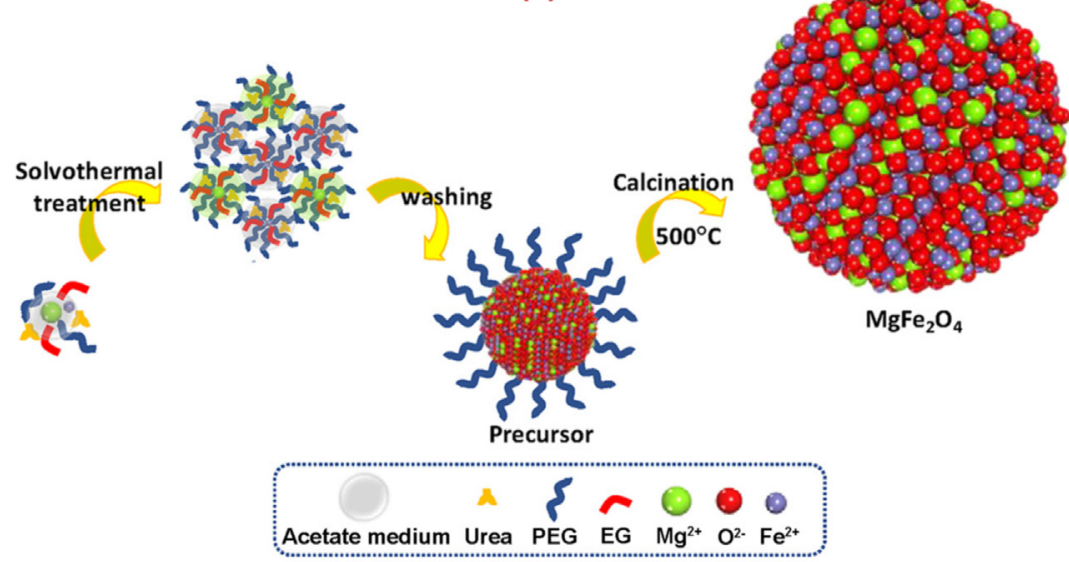

(b)

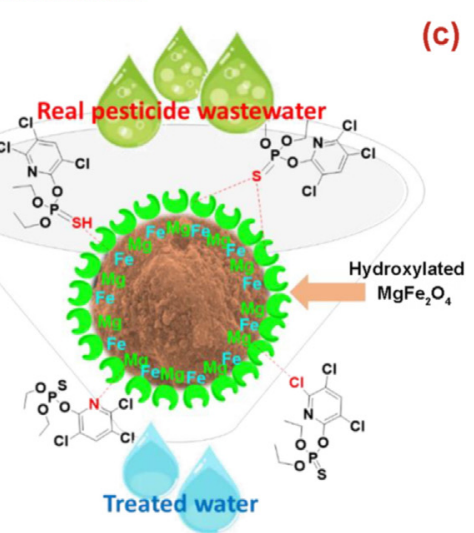

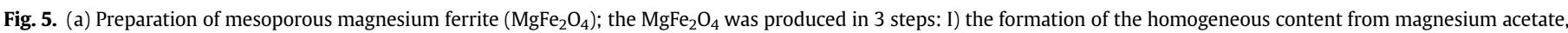

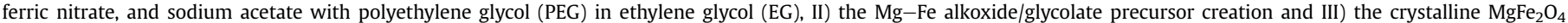

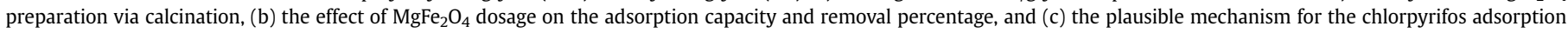
onto the $\mathrm{MgFe}_{2} \mathrm{O}_{4}$ surface. Reprinted with modification with permission from (Sharma and Kakkar, (2018)). 


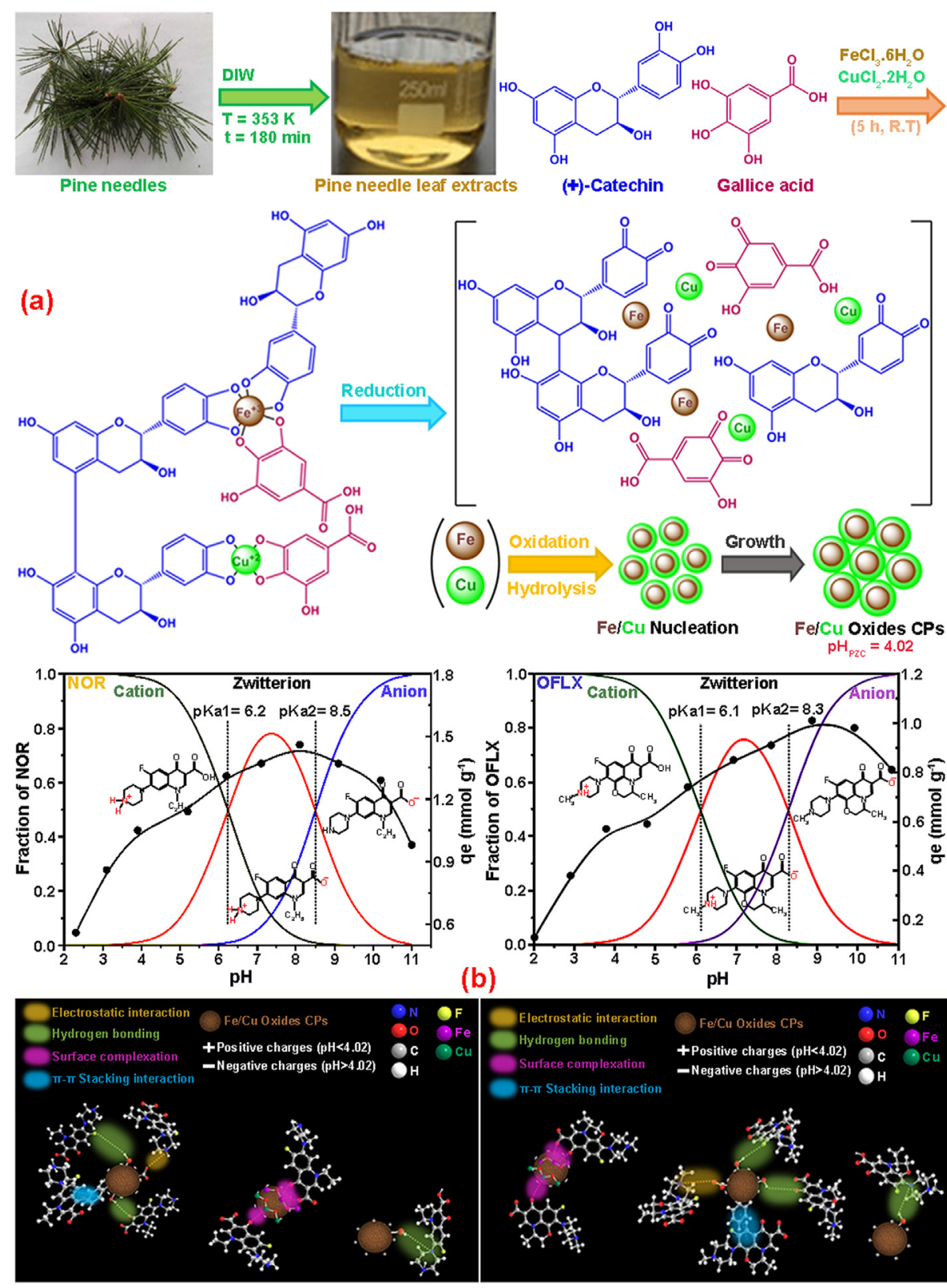

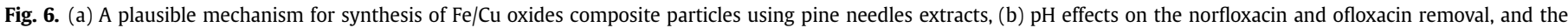
norfloxacin and ofloxacin adsorption mechanism onto Fe/Cu oxides composite particles. Reproduced with modification with permission from (Ma et al., (2020)).

et al., 2019a,b). These unique properties have led to the deployment of carbon-based nanomaterials in diverse fields comprising environmental remediation (Zhang et al., 2019), biotechnology (Molaei, 2019), drug delivery (Panwar et al., 2019), coating (Yi et al., 2019), packaging (Sobhan et al., 2019), and energy storage (Hu et al., 2019), among others (Bi et al., 2019). The carbon-based nanomaterials synthesis for targeted applications is interesting because of their unique properties, including flexibility, superior directionality, high surface area, and outstanding optical properties, which made them special and more challenging than bulk materials for specialized uses (Gan et al., 2019; Nekoueian et al., 2019; M. Yang et al., 2019). The carbon-based nanomaterials cover a range of nano-structural materials and their common forms encompass activated carbon, graphene, graphene oxide, reduced graphene oxide, graphitic carbon nitride $\left(\mathrm{g}-\mathrm{C}_{3} \mathrm{~N}_{4}\right)$, carbon nanotubes, and carbon/graphene quantum dots. Several methods pertaining to the preparation and incorporation of carbon-based nanomaterials for the elimination of contaminants from aqueous media are discussed in this review.

\subsubsection{Activated carbon}

The activated carbon typical elemental composition consists of around $80 \%$ carbon with the rest material comprising hydrogen, nitrogen, oxygen, and sulfur, alongside main functional groups such as phenols, carbonyl, carboxyl, quinones, lactones, and others. Two types of often used activated carbon are powdered activated carbon and granular activated carbon. The pretreatment, carbonization, and activation are the three essential steps during the production of activated carbon. The activated carbon is characterized as carbonrich material with a well-built internal porous structure that is usually created via the pyrolysis and chemical treatment of natural sources, including wood (Danish and Ahmad, 2018), coal (Shokry et al., 2019), rice husks (Menya et al., 2018), sugarcane bagasse 
Table 1

Data on the drugs or pesticides removal by metal oxides and their composites.

\begin{tabular}{|c|c|c|c|c|c|c|}
\hline Adsorbents & Pollutants & $\mathrm{pH}$ & Temperature (K) & Time (min) & $\mathrm{q}_{\max }\left(\mathrm{mg} \mathrm{g}^{-1}\right)$ & Reference \\
\hline $\mathrm{Fe}_{3} \mathrm{O}_{4}$ & Carbamazepine & 7 & 300 & 180 & 0.715 & Misra et al. (2018) \\
\hline $\mathrm{Fe}_{3} \mathrm{O}_{4}$ & Levofloxacin & 6.5 & 298 & 240 & 6.094 & Al-Jabari et al. (2019) \\
\hline $\mathrm{Fe}_{3} \mathrm{O}_{4}$ & Rifampicin & 5.5 & 303 & 60 & 84.8 & Cai et al. (2019) \\
\hline$\alpha-\mathrm{Fe}_{2} \mathrm{O}_{3}$ & Cephalexin & 7.5 & 298 & 180 & 52.28 & Nassar et al. (2018) \\
\hline $\mathrm{Fe}_{2} \mathrm{O}_{3}$ & Carbamazepine & - & 298 & 150 & 2.89 & Rajendran and Sen (2018) \\
\hline$\alpha$-FeOOH & Diclofenac & 5.23 & 298 & 480 & 0.025 & Zhao et al. (2017) \\
\hline$\alpha-\mathrm{FeOOH}$ & Tetracycline & 5.5 & 298 & 5760 & 1.726 & Wu et al. (2019) \\
\hline Ferrihydrite & Tetracycline & 5.5 & 298 & 5760 & 3.822 & Wu et al. (2019) \\
\hline $\mathrm{Fe}_{3} \mathrm{O}_{4} /$ red mud & Ciprofloxacin & 6 & 298 & 180 & 110.15 & Aydin et al. (2019) \\
\hline $\mathrm{Ag} / \mathrm{Fe}_{3} \mathrm{O}_{4}$ & Riboflavin & 6 & 298 & 2 & 18.14 & Akhond et al. (2016) \\
\hline $\mathrm{ZnO}$ & Ciprofloxacin & 4 & 298 & 150 & 8.88 & Dhiman and Sharma (2019a) \\
\hline $\mathrm{MgO}$ & Linezolid & 10 & 308 & - & 123.45 & Fakhri and Behrouz (2015) \\
\hline $\mathrm{ZnO}-\mathrm{MgO}$ & Linezolid & 10 & 308 & - & 140.28 & Fakhri and Behrouz (2015) \\
\hline $\mathrm{CuO}$ & Ciprofloxacin & 4 & 298 & 135 & 8.15 & Sharma and Dhiman (2017) \\
\hline Cobalt oxide & Doxycycline hyclate & 6 & 313 & 90 & 4.484 & Abbas et al. (2019) \\
\hline $\mathrm{NiFe}_{2} \mathrm{O}_{4}$ & Oxcarbazepine & 2.5 & 303 & 20 & 0.103 & Parashar et al. (2020) \\
\hline $\mathrm{Fe} / \mathrm{Cu}$ oxides & Norfloxacin & 8.12 & 293 & 180 & 523.7 & Ma et al. (2020) \\
\hline $\mathrm{Fe} / \mathrm{Cu}$ oxides & Ofloxacin & 8.87 & 293 & 180 & 455.3 & Ma et al. (2020) \\
\hline $\mathrm{Fe}_{3} \mathrm{O}_{4}$ & Methoxychlor & 2 & 298 & 55 & 163.9 & Nayak et al. (2018) \\
\hline $\mathrm{Fe}_{3} \mathrm{O}_{4}$ & Atrazine & 2 & 298 & 55 & 77.5 & Nayak et al. (2018) \\
\hline $\mathrm{MgFe}_{2} \mathrm{O}_{4}$ & Chlorpyrifos & 10 & 295 & 360 & 4461 & Sharma and Kakkar (2018) \\
\hline $\mathrm{ZnO}$ & Naphthalene & 4 & 298 & 40 & 66.80 & Kaur et al. (2017) \\
\hline CTAB-ZnO & Naphthalene & 4 & 298 & 40 & 89.96 & Kaur et al. (2017) \\
\hline BMTF-IL-ZnO & Naphthalene & 4 & 298 & 40 & 148.3 & Kaur et al. (2017) \\
\hline $\mathrm{Hr}-\mathrm{MgO}^{\mathrm{c}}$ & Chlorpyrifos & 7 & 295 & 60 & 3974 & Sharma and Kakkar (2017) \\
\hline $\mathrm{CeO}_{2}$ & 2,4-Dichloro phenoxy acetic acid & - & 308 & 100 & 95.78 & Abdelillah Ali Elhussein et al. (2018) \\
\hline
\end{tabular}

a) CTAB-ZnO: Cetyltrimethylammonium bromide functionalized Zinc oxide.

b) BMTF-IL-ZnO: 1-Butyl-3-methylimidazolium tetrafluoroborate functionalized Zinc oxide.

c) Hr-MgO: Hierarchical magnesium oxide.

(Guo et al., 2020), orange peel (Tovar et al., 2019), bamboo (Negara et al., 2019), date stones (Chowdhury et al., 2019), and other organic wastes (A. Rahman et al., 2019; B. Yang et al., 2019; Yek et al., 2019).

The activated carbons with a broad variety of functional groups, well-organized micro, meso, and macro-pores, and high surface area have been applied as the oldest, most popular, and most common sorbent for the adsorption of organic contaminants in water and wastewater remediation (Mansour et al., 2018; Völker et al., 2019). The main emphasis has been on improving the activation procedures in an optimum manner with proper precursors for different pollutants because the activation method greatly influences pore structure and surface functional groups of activated carbons (Bhatnagar et al., 2013). The activation procedure is typically performed by acidic/basic-, microwave-, ozone-, and plasma treatment wherein the activated carbon surface modification is carried out by physical, chemical, and physiochemical treatment for higher sorption of specific contaminants (Ahmed, 2017). Of note, physical and chemical activation are the two major types of treatments. The physical activation occurs in the presence of inert gases, $\mathrm{O}_{2}$ absence or activation by oxidizing agents such as steam, air, $\mathrm{CO}_{2}$ and their combination, while the chemical activation occurs via carbonization, impregnation with base $\left(\mathrm{KOH}, \mathrm{NaOH}\right.$ and $\left.\mathrm{Na}_{2} \mathrm{CO}_{3}\right)$, alkali metal salt $\left(\mathrm{AlCl}_{3}\right.$ and $\left.\mathrm{ZnCl}_{2}\right)$ or acid $\left(\mathrm{H}_{3} \mathrm{PO}_{4}\right.$ and $\left.\mathrm{H}_{2} \mathrm{SO}_{4}\right)$, and strongly dehydration (Shejale et al., 2020). The activation by using chemical oxidants is performed under mild conditions as compared to physical activation and is created by the chemical-treated activated carbons with higher micro-porosity and larger surface area (Ahmed, 2017). Thus, the source material, activation method, and any additional modifications determine the surface area (up to $2500 \mathrm{~m}^{2} \mathrm{~g}^{-1}$ ) and the accurate internal pore composition of activated carbon.

4.2.1.1. Drugs adsorption onto activated carbons. Many studies have been reported for the adsorption of drug from aqueous solution onto activated carbons, e.g., diclofenac (de Franco et al., 2018) and triclosan (Bernal et al., 2020a) onto the activated carbons, atenolol (Haro et al., 2017), and phenol, salicylic acid, and methylparaben (Bernal et al., 2020b) onto commercial granular activated carbons, ampicillin (Del Vecchio et al., 2019), and acetaminophen and salicylic acid (Bernal et al., 2020c) onto granular activated carbon, ranitidine onto activated carbon prepared from lemon peel (Bhattacharyya et al., 2019), metformin hydrochloride onto Zea mays tassel activated carbon (Kalumpha et al., 2020), metronidazole and sulfamethoxazole onto walnut shell-based activated carbon (Teixeira et al., 2019), etodolac onto microporous activated carbon derived from apricot, peach stones, and almond shell mixture biomasses (Erdem et al., 2020), acetaminophen onto cashew nut shell biomass-derived activated carbons (Geczo et al., 2020), acetylsalicylic acid and sodium diclofenac onto lovegrass (Poaceae) derived activated carbon (Cimirro et al., 2020), and amoxicillin onto activated carbon obtained from the sugar and alcohol industry byproducts (Schultz et al., 2020).

The activated carbon textural properties have been tailored to improve its potential for diclofenac adsorption from aqueous media (Moral-Rodríguez et al., 2019). The activated carbons were prepared as activated carbon $0 \quad\left(\mathrm{~S}_{\mathrm{BET}}=799 \mathrm{~m}^{2} \mathrm{~g}^{-1}\right.$, $\left.\mathrm{V}_{\text {Micro }}=0.31 \mathrm{~cm}^{3} \mathrm{~g}^{-1}\right)$, activated carbon $12\left(\mathrm{~S}_{\mathrm{BET}}=1146 \mathrm{~m}^{2} \mathrm{~g}^{-1}\right.$, $\left.\mathrm{V}_{\text {Micro }}=0.45 \mathrm{~cm}^{3} \mathrm{~g}^{-1}\right)$, activated carbon $24\left(\mathrm{~S}_{\mathrm{BET}}=1279 \mathrm{~m}^{2} \mathrm{~g}^{-1}\right.$, $\left.\mathrm{V}_{\text {Micro }}=0.50 \mathrm{~cm}^{3} \mathrm{~g}^{-1}\right)$ and activated carbon $40\left(\mathrm{~S}_{\mathrm{BET}}=1801 \mathrm{~m}^{2} \mathrm{~g}^{-1}\right.$, $\mathrm{V}_{\text {Micro }}=0.66 \mathrm{~cm}^{3} \mathrm{~g}^{-1}$ ) by modifying a commercial activated carbon via physical treatment with $\mathrm{CO}_{2}$ for $0,12,24$ and $40 \mathrm{~h}$ during the activation process. The $\mathrm{q}_{\max }$ of activated carbons was mainly attributed to the activated carbons porous structure or activated carbons textural properties and was considerably improved by increasing the activation time. Hence, the activated carbonspotential for the removal of emerging pollutants can be significantly improved by tailoring the activated carbons textural properties.

The influence of hydrophobicity/lipophilicity of pharmaceuticals and personal care products (PPCPs), caffeine $\left(\log \mathrm{K}_{\mathrm{ow}}=-0.07\right)$, 
Table 2

The recent drugs and pesticide adsorption onto activated carbons.

\begin{tabular}{|c|c|c|c|c|c|c|c|c|}
\hline $\begin{array}{l}\text { Activation } \\
\text { Method }\end{array}$ & Modification conditions & Carbon source & Adsorbate & Adsorption conditions & $\begin{array}{l}\text { Adsorption } \\
\text { isotherm }\end{array}$ & Adsorption kinetic & $\mathrm{q}_{\max }\left(\mathrm{mg} \mathrm{g}^{-1}\right)$ & Reference \\
\hline $\begin{array}{l}\text { Chemical } \\
\text { activation }\end{array}$ & $\mathrm{KOH}, 4 \mathrm{~h}$ & Prosopis juliflora & Tetracycline & $\begin{array}{l}\mathrm{pH}=7.0 \\
\mathrm{C}_{0}=1 \mathrm{mg} \mathrm{L}^{-1} \\
\mathrm{~V}=100 \mathrm{~mL}, \mathrm{~m}=1 \mathrm{~g} \mathrm{~L}^{-1} \\
\mathrm{~T}=303 \mathrm{~K}, \mathrm{t}=120 \mathrm{~min}\end{array}$ & Langmuir & $\begin{array}{l}\text { Pseudo-second- } \\
\text { order }\end{array}$ & 28.81 & Manjunath et al. (2020) \\
\hline $\begin{array}{l}\text { Chemical } \\
\text { activation }\end{array}$ & $\mathrm{KOH}, 4 \mathrm{~h}$ & Prosopis juliflora & Metronidazole & $\begin{array}{l}\mathrm{pH}=7.0 \\
\mathrm{C}_{0}=1 \mathrm{mg} \mathrm{L}^{-1} \\
\mathrm{~V}=100 \mathrm{~mL}, \mathrm{~m}=1 \mathrm{~g} \mathrm{~L}^{-1} \\
\mathrm{~T}=303 \mathrm{~K}, \mathrm{t}=120 \mathrm{~min}\end{array}$ & Langmuir & $\begin{array}{l}\text { Pseudo-second- } \\
\text { order }\end{array}$ & 25.06 & Manjunath et al. (2020) \\
\hline $\begin{array}{l}\text { Chemical } \\
\text { activation }\end{array}$ & $\mathrm{KOH}, 4 \mathrm{~h}$ & Prosopis juliflora & Sulfadiazine & $\begin{array}{l}\mathrm{pH}=7.0 \\
\mathrm{C}_{0}=1 \mathrm{mg} \mathrm{L}^{-1} \\
\mathrm{~V}=100 \mathrm{~mL}, \mathrm{~m}=1 \mathrm{~g} \mathrm{~L}^{-1} \\
\mathrm{~T}=303 \mathrm{~K}, \mathrm{t}=120 \mathrm{~min}\end{array}$ & Langmuir & $\begin{array}{l}\text { Pseudo-second- } \\
\text { order }\end{array}$ & 18.48 & Manjunath et al. (2020) \\
\hline $\begin{array}{l}\text { Chemical } \\
\text { activation }\end{array}$ & $\begin{array}{l}\text { I) Carbonization }\left(500{ }^{\circ} \mathrm{C}, 2 \mathrm{~h}\right) \\
\text { II) } \mathrm{NaOH}\left(700^{\circ} \mathrm{C}, 90 \mathrm{~min}\right)\end{array}$ & Date press cake & Cefixime & $\begin{array}{l}\mathrm{pH}=4.0 \\
\mathrm{C}_{0}=500 \mathrm{mg} \mathrm{L}^{-1} \\
\mathrm{~V}=25 \mathrm{~mL}, \mathrm{~m}=1 \mathrm{~g} \mathrm{~L}^{-1} \\
\mathrm{~T}=298 \mathrm{~K}, \mathrm{t}=240 \mathrm{~min}\end{array}$ & Redlich-Peterson & $\begin{array}{l}\text { Elovich } \\
\text { Pseudo-second- } \\
\text { order }\end{array}$ & 557.9 & Hasanzadeh et al. (2020) \\
\hline $\begin{array}{l}\text { Chemical } \\
\text { activation }\end{array}$ & $\begin{array}{l}\text { I) Carbonization }\left(500^{\circ} \mathrm{C}, 2 \mathrm{~h}\right) \\
\text { II) } \mathrm{KOH}\left(700^{\circ} \mathrm{C}, 90 \mathrm{~min}\right)\end{array}$ & Date press cake & Cefixime & $\begin{array}{l}\mathrm{pH}=4.0 \\
\mathrm{C}_{0}=500 \mathrm{mg} \mathrm{L}^{-1} \\
\mathrm{~V}=25 \mathrm{~mL}, \mathrm{~m}=1 \mathrm{~g} \mathrm{~L}^{-1} \\
\mathrm{~T}=298 \mathrm{~K}, \mathrm{t}=240 \mathrm{~min}\end{array}$ & Redlich-Peterson & $\begin{array}{l}\text { Elovich } \\
\text { Pseudo-second- } \\
\text { order }\end{array}$ & 571.5 & Hasanzadeh et al. (2020) \\
\hline $\begin{array}{l}\text { Chemical } \\
\text { activation }\end{array}$ & $\begin{array}{l}\text { I) Steam-pyrolysis }\left(90{ }^{\circ} \mathrm{C}, 3 \mathrm{~h}\right) \\
\text { II) } \mathrm{HNO}_{3}+\mathrm{H}_{2} \mathrm{O}_{2}\left(200^{\circ} \mathrm{C}, 1 \mathrm{~h}\right)\end{array}$ & $\begin{array}{l}\text { Sucrose } \\
\text { Polystyrene }\end{array}$ & Tetracycline & $\begin{array}{l}\mathrm{pH}=7.0 \\
\mathrm{C}_{0}=60 \mathrm{mg} \mathrm{L}-1 \\
\mathrm{~V}=25 \mathrm{~mL}, \mathrm{~m}=0.1 \mathrm{~g} \\
\mathrm{~T}=298 \mathrm{~K}, \mathrm{t}=120 \mathrm{~min}\end{array}$ & Langmuir & $\begin{array}{l}\text { Pseudo-second- } \\
\text { order }\end{array}$ & 909.2 & Hubetska et al. (2020) \\
\hline $\begin{array}{l}\text { Chemical } \\
\text { activation }\end{array}$ & $\begin{array}{l}\text { I) Carbonization }\left(300^{\circ} \mathrm{C}\right) \\
\text { II) Impregnation }\left(\mathrm{H}_{3} \mathrm{PO}_{4}, 24 \mathrm{~h}\right) \\
\text { III) Chemical activation }\left(400{ }^{\circ} \mathrm{C}\right)\end{array}$ & Zea mays tassel & Metformin hydrochloride & $\begin{array}{l}\mathrm{pH}=9.5 \\
\mathrm{C}_{0}=152.5 \mathrm{mg} \mathrm{L}-1 \\
\mathrm{~V}=20 \mathrm{~mL}, \mathrm{~m}=0.575 \mathrm{~g} \\
\mathrm{~T}=293 \mathrm{~K}, \mathrm{t}=67.5 \mathrm{~min}\end{array}$ & Langmuir & $\begin{array}{l}\text { Pseudo-second- } \\
\text { order }\end{array}$ & 44.84 & Kalumpha et al. (2020) \\
\hline $\begin{array}{l}\text { Chemical } \\
\text { activation }\end{array}$ & $\begin{array}{l}\text { I) Impregnation }\left(\mathrm{FeSO}_{4}, 700{ }^{\circ} \mathrm{C}\right) \\
\text { II) Pyrolysis }\left(700^{\circ} \mathrm{C}, 2 \mathrm{~h}\right)\end{array}$ & $\begin{array}{l}\text { Apricot and peach } \\
\text { stones } \\
+ \\
\text { Almond shell }\end{array}$ & Etodolac & $\begin{array}{l}\mathrm{pH}=4.0 \\
\mathrm{C}_{0}=20 \mathrm{mg} \mathrm{L}^{-1} \\
\mathrm{~V}=100 \mathrm{~mL}, \mathrm{~m}=5 \mathrm{~g} \mathrm{~L}^{-1} \\
\mathrm{~T}=298 \mathrm{~K}, \mathrm{t}=150 \mathrm{~min}\end{array}$ & Freundlich & $\begin{array}{l}\text { Pseudo-second- } \\
\text { order }\end{array}$ & 19.74 & Erdem et al. (2020) \\
\hline $\begin{array}{l}\text { Physical } \\
\text { activation }\end{array}$ & Physically activated with $\mathrm{CO}_{2}$ & Coconut shell & Methylparaben & $\begin{array}{l}\mathrm{pH}=7.42 \\
\mathrm{C}_{0}=6.58 \mathrm{mmol} \mathrm{L} \\
\mathrm{V}=25 \mathrm{~mL}, \mathrm{~m}=100 \mathrm{mg} \\
\mathrm{T}=293 \mathrm{~K}, \mathrm{t}=7 \text { days }\end{array}$ & Langmuir & - & $1.58 \mathrm{mmol} \mathrm{g}^{-1}$ & Bernal et al. (2020b) \\
\hline $\begin{array}{l}\text { Chemical } \\
\text { activation }\end{array}$ & $\begin{array}{l}\text { I) } \mathrm{H}_{3} \mathrm{PO}_{4}\left(85^{\circ} \mathrm{C}, 2 \mathrm{~h}\right) \\
\text { II) Carbonization }\left(400-700{ }^{\circ} \mathrm{C} \text {, }\right. \\
\text { 2h) }\end{array}$ & Cashew nut shell & Acetaminophen & $\begin{array}{l}\mathrm{pH}=-\mathrm{C}_{0}=50 \mathrm{mg} \mathrm{L}^{-1} \\
\mathrm{~V}=25 \mathrm{~mL}, \mathrm{~m}=12.5 \mathrm{mg} \\
\mathrm{T}=-\mathrm{K}, \mathrm{t}=24 \mathrm{~h}\end{array}$ & Langmuir & $\begin{array}{l}\text { Pseudo-second- } \\
\text { order }\end{array}$ & 146 & Geczo et al. (2020) \\
\hline $\begin{array}{l}\text { Chemical } \\
\text { activation }\end{array}$ & $\begin{array}{l}\text { I) Impregnation }\left(\mathrm{ZnCl}_{2}, 80^{\circ} \mathrm{C} \text {, }\right. \\
2 \mathrm{~h}) \\
\text { II) Pyrolysis }\left(700^{\circ} \mathrm{C}, 1 \mathrm{~h}\right)\end{array}$ & Lovegrass (Poaceae) & Sodium diclofenac & $\begin{array}{l}\mathrm{pH}=4.0 \\
\mathrm{C}_{0}=200 \mathrm{mg} \mathrm{L}^{-1} \\
\mathrm{~V}=20 \mathrm{~mL}, \mathrm{~m}=60 \mathrm{mg} \\
\mathrm{T}=298 \mathrm{~K}, \mathrm{t}=60 \mathrm{~min}\end{array}$ & Liu & $\begin{array}{l}\text { Pseudo-second- } \\
\text { order }\end{array}$ & 312.4 & Cimirro et al. (2020) \\
\hline $\begin{array}{l}\text { Chemical } \\
\text { activation }\end{array}$ & $\begin{array}{l}\text { I) Impregnation }\left(\mathrm{ZnCl}_{2}, 80^{\circ} \mathrm{C} \text {, }\right. \\
2 \mathrm{~h}) \\
\text { II) Pyrolysis }\left(700^{\circ} \mathrm{C}, 1 \mathrm{~h}\right)\end{array}$ & Lovegrass (Poaceae) & Acetylsalicylic acid & $\begin{array}{l}\mathrm{pH}=4.0 \\
\mathrm{C}_{0}=200 \mathrm{mg} \mathrm{L}^{-1} \\
\mathrm{~V}=20 \mathrm{~mL}, \mathrm{~m}=60 \mathrm{mg} \\
\mathrm{T}=298 \mathrm{~K}, \mathrm{t}=45 \mathrm{~min}\end{array}$ & Liu & $\begin{array}{l}\text { Pseudo-second- } \\
\text { order }\end{array}$ & 221.7 & Cimirro et al. (2020) \\
\hline $\begin{array}{l}\text { Chemical } \\
\text { activation }\end{array}$ & $\begin{array}{l}\text { I) Impregnation }\left(\mathrm{H}_{3} \mathrm{PO}_{4}, 24 \mathrm{~h}\right) \\
\text { II) Microwave }\left(\mathrm{N}_{2}, 20 \mathrm{~min} \text {, }\right. \\
1 \mathrm{~kW})\end{array}$ & Reynoutria japonica & Diclofenac & $\begin{array}{l}\mathrm{pH}=7.0 \\
\mathrm{C}_{0}=300 \mathrm{mg} \mathrm{L} \\
\mathrm{V}=10 \mathrm{~mL}, \mathrm{~m}=20 \mathrm{mg} \\
\mathrm{T}=296 \mathrm{~K}, \mathrm{t}=90 \mathrm{~min}\end{array}$ & Sips & $\begin{array}{l}\text { Pseudo-second- } \\
\text { order }\end{array}$ & 87.09 & Koutník et al. (2020) \\
\hline $\begin{array}{l}\text { Chemical } \\
\text { activation }\end{array}$ & $\begin{array}{l}\text { I) Impregnation }\left(\mathrm{H}_{3} \mathrm{PO}_{4}, 24 \mathrm{~h}\right) \\
\text { II) Microwave }\left(\mathrm{N}_{2}, 20 \mathrm{~min} \text {, }\right. \\
1 \mathrm{~kW})\end{array}$ & Reynoutria japonica & Paracetamol & $\begin{array}{l}\mathrm{pH}=7.0 \\
\mathrm{C}_{0}=300 \mathrm{mg} \mathrm{L}^{-1} \\
\mathrm{~V}=10 \mathrm{~mL}, \mathrm{~m}=20 \mathrm{mg} \\
\mathrm{T}=296 \mathrm{~K}, \mathrm{t}=90 \mathrm{~min}\end{array}$ & Sips & $\begin{array}{l}\text { Pseudo-second- } \\
\text { order }\end{array}$ & 136.61 & Koutník et al. (2020) \\
\hline
\end{tabular}




\begin{tabular}{|c|c|c|c|c|c|c|c|c|}
\hline $\begin{array}{l}\text { Activation } \\
\text { Method }\end{array}$ & Modification conditions & Carbon source & Adsorbate & Adsorption conditions & $\begin{array}{l}\text { Adsorption } \\
\text { isotherm }\end{array}$ & Adsorption kinetic & $\mathrm{q}_{\max }\left(\mathrm{mg} \mathrm{g}^{-1}\right)$ & Reference \\
\hline $\begin{array}{l}\text { Chemical } \\
\text { activation }\end{array}$ & $\begin{array}{l}\text { I) Impregnation }\left(\mathrm{ZnCl}_{2} \text {, R.T., }\right. \\
24 \mathrm{~h}) \\
\text { II) Carbonization }\left(500{ }^{\circ} \mathrm{C}, 2 \mathrm{~h}\right)\end{array}$ & Sugar cane bagasse & Diclofenac sodium & $\begin{array}{l}\mathrm{pH}=2.0 \\
\mathrm{C}_{0}=50 \mathrm{mg} \mathrm{L}^{-1} \\
\mathrm{~V}=25 \mathrm{~mL}, \mathrm{~m}=0.4 \mathrm{~g} \mathrm{~L}^{-1} \\
\mathrm{~T}=298 \mathrm{~K}, \mathrm{t}=15 \mathrm{~min}\end{array}$ & Langmuir & $\begin{array}{l}\text { Pseudo-second- } \\
\text { order }\end{array}$ & 315.0 & Abo El Naga et al. (2019) \\
\hline $\begin{array}{l}\text { Chemical } \\
\text { activation }\end{array}$ & $\begin{array}{l}\text { I) Pyrolysis }\left(300{ }^{\circ} \mathrm{C}, 1 \mathrm{~h}, \mathrm{~N}_{2}\right) \\
\text { II) Impregnation }\left(\mathrm{K}_{2} \mathrm{CO}_{3}, 105^{\circ} \mathrm{C} \text {, }\right. \\
8 \mathrm{~h}) \\
\text { III) Carbonization }\left(900{ }^{\circ} \mathrm{C}, 1 \mathrm{~h} \text {, }\right. \\
\left.\mathrm{N}_{2}\right)\end{array}$ & Walnut shell & Metronidazole & $\begin{array}{l}\mathrm{pH}=6.0 \\
\mathrm{C}_{0}=40 \mathrm{mg} \mathrm{L} \\
\mathrm{V}=-\mathrm{mL}, \mathrm{m}=10 \mathrm{mg} \\
\mathrm{T}=293 \mathrm{~K}, \mathrm{t}=48 \mathrm{~h}\end{array}$ & Langmuir & - & 107.4 & Teixeira et al. (2019) \\
\hline $\begin{array}{l}\text { Chemical } \\
\text { activation }\end{array}$ & $\begin{array}{l}\text { I) Pyrolysis }\left(300{ }^{\circ} \mathrm{C}, 1 \mathrm{~h}, \mathrm{~N}_{2}\right) \\
\text { II) Impregnation }\left(\mathrm{K}_{2} \mathrm{CO}_{3}, 105^{\circ} \mathrm{C} \text {, }\right. \\
8 \mathrm{~h}) \\
\text { III) Carbonization }\left(900{ }^{\circ} \mathrm{C}, 1 \mathrm{~h} \text {, }\right. \\
\left.\mathrm{N}_{2}\right)\end{array}$ & Walnut shell & Sulfamethoxazole & $\begin{array}{l}\mathrm{pH}=6.0 \\
\mathrm{C}_{0}=40 \mathrm{mg} \mathrm{L} \\
\mathrm{V}=-\mathrm{mL}, \mathrm{m}=10 \mathrm{mg} \\
\mathrm{T}=293 \mathrm{~K}, \mathrm{t}=48 \mathrm{~h}\end{array}$ & Langmuir & - & 93.5 & Teixeira et al. (2019) \\
\hline $\begin{array}{l}\text { Chemical } \\
\text { activation }\end{array}$ & $\begin{array}{l}\text { I) Carbonization }\left(500^{\circ} \mathrm{C}, 2 \mathrm{~h}, \mathrm{~N}_{2}\right) \\
\text { II) Impregnation }\left(\mathrm{KOH}, 120^{\circ} \mathrm{C}\right) \\
\text { III) Pyrolysis }\left(550^{\circ} \mathrm{C}, 1 \mathrm{~h}, \mathrm{~N}_{2}\right)\end{array}$ & Date palm leaflets & Chlorpheniramine & $\begin{array}{l}\mathrm{pH}=7.0 \\
\mathrm{C}_{0}=50 \mathrm{mg} \mathrm{L} \\
\mathrm{V}=25 \mathrm{~mL}, \mathrm{~m}=0.06 \mathrm{~g} \\
\mathrm{~T}=298 \mathrm{~K}, \mathrm{t}=48 \mathrm{~h}\end{array}$ & Langmuir & $\begin{array}{l}\text { Pseudo-second- } \\
\text { order }\end{array}$ & 100.2 & (S. N. F. Ali et al., 2019) \\
\hline $\begin{array}{l}\text { Chemical } \\
\text { activation }\end{array}$ & $\begin{array}{l}\text { I) Carbonization }\left(500{ }^{\circ} \mathrm{C}, 2 \mathrm{~h}, \mathrm{~N}_{2}\right) \\
\text { II) Impregnation }\left(\mathrm{KOH}, 120^{\circ} \mathrm{C}\right) \\
\text { III) Pyrolysis }\left(550^{\circ} \mathrm{C}, 1 \mathrm{~h}, \mathrm{~N}_{2}\right)\end{array}$ & Date palm leaflets & Ibuprofen & $\begin{array}{l}\mathrm{pH}=7.0 \\
\mathrm{C}_{0}=50 \mathrm{mg} \mathrm{L} \\
\mathrm{V}=25 \mathrm{~mL}, \mathrm{~m}=0.06 \mathrm{~g} \\
\mathrm{~T}=298 \mathrm{~K}, \mathrm{t}=48 \mathrm{~h}\end{array}$ & Langmuir & $\begin{array}{l}\text { Pseudo-second- } \\
\text { order }\end{array}$ & 52.4 & (S. N. F. Ali et al., 2019) \\
\hline- & - & Babassu coconut & Diclofenac & $\begin{array}{l}\mathrm{pH}=7.0 \\
\mathrm{C}_{0}=50 \mathrm{mg} \mathrm{L} \\
\mathrm{V}=50 \mathrm{~mL}, \mathrm{~m}=0.05 \mathrm{~g} \\
\mathrm{~T}=298 \mathrm{~K}, \mathrm{t}=30 \mathrm{~h}\end{array}$ & Freundlich & $\begin{array}{l}\text { Intraparticle } \\
\text { diffusion }\end{array}$ & 71.150 & Viotti et al. (2019) \\
\hline $\begin{array}{l}\text { Chemical } \\
\text { activation }\end{array}$ & $\begin{array}{l}\text { I) Impregnation with } \mathrm{ZnCl}_{2} \\
\text { II) Pyrolysis }\left(600^{\circ} \mathrm{C}, 30 \mathrm{~min}, \mathrm{~N}_{2}\right) \\
\text { III) Leaching out with } \mathrm{HCl} \\
\text { (Reflux, } 1 \mathrm{~h} \text { ) }\end{array}$ & Brazil nutshells & Acetaminophen & $\begin{array}{l}\mathrm{pH}=7.0 \\
\mathrm{C}_{0}=800 \mathrm{mg} \mathrm{L}^{-1} \\
\mathrm{~V}=-\mathrm{mL}, \mathrm{m}=1.5 \mathrm{~g} \mathrm{~L}^{-1} \\
\mathrm{~T}=298 \mathrm{~K}, \mathrm{t}=30 \mathrm{~min}\end{array}$ & Liu & $\begin{array}{l}\text { Avrami fractional- } \\
\text { order }\end{array}$ & 411.0 & Lima et al. (2019) \\
\hline $\begin{array}{l}\text { Chemical } \\
\text { activation }\end{array}$ & $\begin{array}{l}\text { I) Impregnation }\left(\mathrm{H}_{3} \mathrm{PO}_{4}, 85^{\circ} \mathrm{C} \text {, }\right. \\
\text { 2h) } \\
\text { II) Pyrolysis }\left(500{ }^{\circ} \mathrm{C}, 2 \mathrm{~h}, \mathrm{~N}_{2}\right)\end{array}$ & Kenaf & Paracetamol & $\begin{array}{l}\mathrm{pH}=7.0 \\
\mathrm{C}_{0}=120 \mathrm{mg} \mathrm{L} \\
\mathrm{V}=15 \mathrm{~mL}, \mathrm{~m}=10 \mathrm{mg} \\
\mathrm{T}=298 \mathrm{~K}, \mathrm{t}=300 \mathrm{~min}\end{array}$ & - & $\begin{array}{l}\text { Pseudo-second- } \\
\text { order }\end{array}$ & - & $\begin{array}{l}\text { Macías-García et al. } \\
\text { (2019) }\end{array}$ \\
\hline $\begin{array}{l}\text { Chemical } \\
\text { activation }\end{array}$ & $\begin{array}{l}\text { I) Mechanical grinding } \\
\text { II) Heat }\left(105^{\circ} \mathrm{C}, 4 \mathrm{~h}\right) \\
\text { III) Carbonization }\left(350-500{ }^{\circ} \mathrm{C} \text {, }\right. \\
\text { 1h) } \\
\text { IV) Impregnation }\left(\mathrm{NaOH}, 130^{\circ} \mathrm{C} \text {, }\right. \\
\text { 4h) }\end{array}$ & Waste lemon peel & Ranitidine & $\begin{array}{l}\mathrm{pH}=6.0 \\
\mathrm{C}_{0}=10 \mathrm{mg} \mathrm{L} \\
\mathrm{V}=100 \mathrm{~mL}, \mathrm{~m}=200 \mathrm{mg} \\
\mathrm{T}=313 \mathrm{~K}, \mathrm{t}=60 \mathrm{~min}\end{array}$ & - & $\begin{array}{l}\text { Pseudo-second- } \\
\text { order }\end{array}$ & - & $\begin{array}{l}\text { Bhattacharyya et al. } \\
\text { (2019) }\end{array}$ \\
\hline $\begin{array}{l}\text { Chemical } \\
\text { activation }\end{array}$ & $\begin{array}{l}\text { I) Carbonization }\left(500{ }^{\circ} \mathrm{C}, 2 \mathrm{~h}, \mathrm{~N}_{2}\right) \\
\text { II) Impregnation }\left(\mathrm{NaOH}, 800^{\circ} \mathrm{C} \text {, }\right. \\
\text { 1h) } \\
\text { III) Dehydration }\left(105^{\circ} \mathrm{C}, 24 \mathrm{~h}\right)\end{array}$ & Rice husk & Acetaminophen & $\begin{array}{l}\mathrm{pH}=5.8 \\
\mathrm{C}_{0}=40 \mathrm{mg} \mathrm{L}-1 \\
\mathrm{~V}=100 \mathrm{~mL}, \mathrm{~m}=0.15 \mathrm{~g} \\
\mathrm{~T}=298 \mathrm{~K}, \mathrm{t}=60 \mathrm{~min}\end{array}$ & Redlich-Peterson & $\begin{array}{l}\text { Pseudo-second- } \\
\text { order }\end{array}$ & 50.25 & $\begin{array}{l}\text { Paredes-Laverde et al. } \\
\text { (2019) }\end{array}$ \\
\hline $\begin{array}{l}\text { Chemical } \\
\text { activation }\end{array}$ & $\begin{array}{l}\text { I) Impregnation }\left(\mathrm{ZnCl}_{2}, 28^{\circ} \mathrm{C} \text {, }\right. \\
\text { 24h) } \\
\text { II) Carbonization }\left(600^{\circ} \mathrm{C}, 2 \mathrm{~h}, \mathrm{~N}_{2}\right)\end{array}$ & Tea waste & Sodium diclofenac & $\begin{array}{l}\mathrm{pH}=6.5 \\
\mathrm{C}_{0}=30 \mathrm{mg} \mathrm{L}^{-1} \\
\mathrm{~V}=100 \mathrm{~mL}, \mathrm{~m}=300 \mathrm{mg} \mathrm{L}^{-1} \\
\mathrm{~T}=303 \mathrm{~K}, \mathrm{t}=8 \mathrm{~h}\end{array}$ & Langmuir & $\begin{array}{l}\text { Pseudo-second- } \\
\text { order }\end{array}$ & 91.2 & Malhotra et al. (2018) \\
\hline $\begin{array}{l}\text { Chemical } \\
\text { activation }\end{array}$ & $\begin{array}{l}\text { I) Impregnation with } \mathrm{ZnCl}_{2} \\
(\mathrm{r}=1.5) \\
\text { II) Carbonization }\left(600{ }^{\circ} \mathrm{C}\right)\end{array}$ & $\begin{array}{l}\text { Caesalpinia ferrea seed } \\
\text { pod }\end{array}$ & Captopril & $\begin{array}{l}\mathrm{pH}=7.0 \\
\mathrm{C}_{0}=600 \mathrm{mg} \mathrm{L}^{-1} \\
\mathrm{~V}=20 \mathrm{~mL}, \mathrm{~m}=30 \mathrm{mg} \mathrm{L}^{-1} \\
\mathrm{~T}=298 \mathrm{~K}, \mathrm{t}=60 \mathrm{~min}\end{array}$ & Liu & $\begin{array}{l}\text { General-order } \\
\text { kinetic }\end{array}$ & 535.5 & Kasperiski et al. (2018) \\
\hline $\begin{array}{l}\text { Chemical } \\
\text { treatment }\end{array}$ & $\begin{array}{l}\text { I) Dehydration }\left(105^{\circ} \mathrm{C}, 24 \mathrm{~h}\right) \\
\text { II) Carbonization }\left(600^{\circ} \mathrm{C}, 1 \mathrm{~h}\right) \\
\text { III) Chemical-thermal activation }\end{array}$ & $\begin{array}{l}\text { Quercus Brantii (oak) } \\
\text { acorn }\end{array}$ & Acetaminophen & $\begin{array}{l}\mathrm{pH}=3.0 \\
\mathrm{C}_{0}=100 \mathrm{mg} \mathrm{L} \\
\mathrm{V}=100 \mathrm{~mL}, \mathrm{~m}=100 \mathrm{mg} \\
\mathrm{T}=298 \mathrm{~K}, \mathrm{t}=150 \mathrm{~min}\end{array}$ & Freundlich & $\begin{array}{l}\text { Pseudo-second- } \\
\text { order }\end{array}$ & 45.45 & Nourmoradi et al. (2018) \\
\hline
\end{tabular}


$\mathrm{pH}=3.0$
$\mathrm{C}_{0}=100 \mathrm{mg}$
$\mathrm{V}=100 \mathrm{~m}, \mathrm{~m}$

$\mathrm{T}=298 \mathrm{~K}, \mathrm{t}=120 \mathrm{~min}$

$\mathrm{pH}=7.0$

$\mathrm{C}_{0}=50 \mathrm{mg} \mathrm{L}^{-1}$

Chemical
activation

Impregnation with $\mathrm{FeCl}_{3}$

Tara gum

Antipyrine

Chemical activation

Carbonization $\left(700^{\circ} \mathrm{C}, 1 \mathrm{~h}, \mathrm{~N}_{2}\right)$ Pomegranate wood I) Impregnation $\left(\mathrm{NH}_{4} \mathrm{Cl}, 105^{\circ} \mathrm{C}\right.$

III) Pyrolysis $\left(800{ }^{\circ} \mathrm{C}, 2.5 \mathrm{~h}, \mathrm{~N}_{2}\right)$

Chemical

I) Imp

Luffa sponge

II) Carbonization $\left(450^{\circ} \mathrm{C}, 2 \mathrm{~h}\right)$

III) Dehydration $\left(70^{\circ} \mathrm{C}, 24 \mathrm{~h}\right)$

Chemical

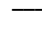

Apricot shells

activation

Walnut shells

Chemical

Chemical

activation

Chemical

activation

Carbonization $\left(950^{\circ} \mathrm{C}, 3 \mathrm{~h}\right) \quad$ Casuarina wood

II) $\mathrm{HCl}$ treatment

Chemical I) Impregnation $\left(\mathrm{KOH}, 105^{\circ} \mathrm{C}\right.$, Orange peel

II) Carbonization $\left(700^{\circ} \mathrm{C}, 2 \mathrm{~h}, \mathrm{~N}_{2}\right)$

Chemical I) Impregnation $\left(\mathrm{KOH}, 105^{\circ} \mathrm{C}\right.$, Orange peel

activation $1 \mathrm{~h}$ )

II) Carbonization $\left(700^{\circ} \mathrm{C}, 2 \mathrm{~h}, \mathrm{~N}_{2}\right)$

Chemical I) Impregnation $\left(\mathrm{KOH}, 105^{\circ} \mathrm{C}\right.$, Orange peel

activation $1 \mathrm{~h})$

II) Carbonization $\left(700^{\circ} \mathrm{C}, 2 \mathrm{~h}, \mathrm{~N}_{2}\right)$

Chemical I) Impregnation $\left(\mathrm{KOH}, 105^{\circ} \mathrm{C}\right.$, Orange peel activation $1 \mathrm{~h}$ )

II) Carbonization $\left(700^{\circ} \mathrm{C}, 2 \mathrm{~h}, \mathrm{~N}_{2}\right)$

Chemical I) Impregnation $\left(\mathrm{KOH}, 105^{\circ} \mathrm{C}, \quad\right.$ Orange peel activation

II) Carbonization $\left(700^{\circ} \mathrm{C}, 2 \mathrm{~h}, \mathrm{~N}_{2}\right)$

Ofloxacin

Atrazine

Atrazine

Atrazine

Chlorothalon

2-(2,4-Dichlorophenoxy)

propionic acid

2-Methyl-4-chlorophenoxy

acetic acid

2,4,5-Trichlorophenoxy acetic

2-Methyl-4-chlorophenox acetic acid

\section{Methyl}

acid

$\mathrm{V}=-\mathrm{mL}, \mathrm{m}=0.2 \mathrm{~g} \mathrm{~L}^{-1}$

$\mathrm{T}=290 \mathrm{~K}, \mathrm{t}=60 \mathrm{~min}$

$\mathrm{pH}=6.0$

$\mathrm{C}_{0}=100 \mathrm{mg} \mathrm{L}^{-1}$

$=100 \mathrm{~mL}, \mathrm{~m}=0.2 \mathrm{~g} \mathrm{~L}^{-1}$

$\mathrm{T}=293 \mathrm{~K}, \mathrm{t}=60 \mathrm{~min}$

$\mathrm{pH}=6.0$

$\mathrm{C}_{0}=30 \mathrm{mg} \mathrm{L}^{-1}$

$\mathrm{V}=50 \mathrm{~mL}, \mathrm{~m}=0.5 \mathrm{~g} \mathrm{~L}^{-1}$

$\mathrm{T}=308 \mathrm{~K}, \mathrm{t}=60 \mathrm{~min}$

$\mathrm{pH}=6.0$

$\mathrm{C}_{0}=90 \mathrm{mg} \mathrm{L}^{-1}$

$\mathrm{V}=50 \mathrm{~mL}, \mathrm{~m}=20 \mathrm{mg}$

$\mathrm{T}=293 \mathrm{~K}, \mathrm{t}=5 \mathrm{~h}$

$\mathrm{pH}=6.0$

$\mathrm{V}=50 \mathrm{~mL}, \mathrm{~m}=20 \mathrm{mg}$

$\mathrm{T}=293 \mathrm{~K}, \mathrm{t}=5 \mathrm{~h}$

$\mathrm{pH}=6.0$

$\mathrm{C}_{0}=90 \mathrm{mg} \mathrm{L}^{-1}$

$\mathrm{V}=50 \mathrm{~mL}, \mathrm{~m}=20 \mathrm{mg}$

$\mathrm{T}=293 \mathrm{~K}, \mathrm{t}=5 \mathrm{~h}$

$\mathrm{pH}=7.0$

$\mathrm{C}_{0}=480 \mathrm{mg} \mathrm{L}^{-1}$

$\mathrm{V}=200 \mathrm{~mL}, \mathrm{~m}=0.5 \mathrm{~g} \mathrm{~L}^{-1}$

$\mathrm{T}=298 \mathrm{~K}, \mathrm{t}=40 \mathrm{~min}$

$\mathrm{pH}=3.0$

$\mathrm{C}_{0}=50 \mathrm{mg} \mathrm{L}^{-1}$

$\mathrm{V}=50 \mathrm{~mL}, \mathrm{~m}=50 \mathrm{mg}$

$\mathrm{T}=313 \mathrm{~K}, \mathrm{t}=210 \mathrm{~min}$

$\mathrm{pH}=3.0$

$\mathrm{C}_{0}=50 \mathrm{mg} \mathrm{L}^{-1}$

$=50 \mathrm{~mL}, \mathrm{~m}=50 \mathrm{mg}$

$=313 \mathrm{~K}, \mathrm{t}=210 \mathrm{~min}$

$\mathrm{pH}=3.0$

$\mathrm{V}=50 \mathrm{~mL}, \mathrm{~m}=50 \mathrm{mg}$

$\mathrm{T}=313 \mathrm{~K}, \mathrm{t}=210 \mathrm{~min}$

$\mathrm{pH}=3.0$

$\mathrm{C}_{0}=50 \mathrm{mg} \mathrm{L} \mathrm{L}^{-1}$

$\mathrm{V}=50 \mathrm{~mL}, \mathrm{~m}=50 \mathrm{mg}$

$\mathrm{T}=313 \mathrm{~K}, \mathrm{t}=210 \mathrm{~min}$

$\mathrm{C}_{0}=50 \mathrm{mg}$

$\mathrm{V}=50 \mathrm{~mL}, \mathrm{~m}=50 \mathrm{mg}$

$\mathrm{T}=313 \mathrm{~K}, \mathrm{t}=210 \mathrm{~min}$

$\mathrm{pH}=4.8$

$\mathrm{C}_{0}=5 \mathrm{mg} \mathrm{L}^{-1}$

$\mathrm{V}=50 \mathrm{~mL} \mathrm{~m}=1 \mathrm{~g} \mathrm{~L}^{-1}$

$\mathrm{T}=298 \mathrm{~K}, \mathrm{t}=20 \mathrm{~min}$

$\mathrm{C}_{0}=5 \mathrm{mg} \mathrm{L}^{-1}$
Pseudo-se

orde

96.15

Nourmoradi et al. (2018)

Langmuir

Hyperbolic

307.7

Bedia et al. (2018)

Redlich-Peterson Ritchie second

482.47

orde

Pseudo-second

order

Freundlic

Pseudo-second

order

Freundlich

Pseudo-second-

46.30

order

Freundlich

order

Freundlich

Pseuder

Freundlich

Pse

Langmuir

Pseu

order

Langmuir

Pse

order

Langmuir

Pse

Langmuir

Pse

order

Langmuir

Pseudo-s

order

Langmuir

Pseud

order

Pseudo-second-

order
Alahabadi et al. (2017)

Kong et al. (2017)

Wei et al. (2018)

Wei et al. (2018)

Wei et al. (2018)

Gar Alalm and Nas (2018)

Pandiarajan et al. (2018

Pandiarajan et al. (2018)

Pandiarajan et al. (2018)

Pandiarajan et al. (2018)

Pandiarajan et al. (2018

Hgeig et al. (2019)

Hgeig et al. (2019)

continued on next page) 


\begin{tabular}{|c|c|c|c|c|c|c|c|c|}
\hline $\begin{array}{l}\text { Activation } \\
\text { Method }\end{array}$ & Modification conditions & Carbon source & Adsorbate & Adsorption conditions & $\begin{array}{l}\text { Adsorption } \\
\text { isotherm }\end{array}$ & Adsorption kinetic & $\mathrm{q}_{\max }\left(\mathrm{mg} \mathrm{g}^{-1}\right)$ & Reference \\
\hline $\begin{array}{l}\text { Chemical } \\
\text { activation }\end{array}$ & $\begin{array}{l}\text { I) Impregnation with } \mathrm{Na}_{2} \mathrm{CO}_{3} \\
\text { II) Pre-carbonization }\left(300^{\circ} \mathrm{C} \text {, }\right. \\
\text { 2h) } \\
\text { III) Impregnation }\left(\mathrm{KOH}, 400{ }^{\circ} \mathrm{C} \text {, }\right. \\
\text { 2h) }\end{array}$ & Starch & Pyraclostrobin & $\begin{array}{l}\mathrm{V}=50 \mathrm{~mL}, \mathrm{~m}=1 \mathrm{~g} \mathrm{~L}^{-1} \\
\mathrm{~T}=298 \mathrm{~K}, \mathrm{t}=20 \mathrm{~min} \\
\mathrm{pH}=7.0 \\
\mathrm{C}_{0}=0.5 \mathrm{mg} \mathrm{L}^{-1} \\
\mathrm{~V}=5 \mathrm{~mL}, \mathrm{~m}=50 \mathrm{mg} \\
\mathrm{T}=298 \mathrm{~K}, \mathrm{t}=5 \mathrm{~min}\end{array}$ & Langmuir & $\begin{array}{l}\text { Pseudo-second- } \\
\text { order }\end{array}$ & 66.2 & Suo et al. (2019) \\
\hline $\begin{array}{l}\text { Chemical } \\
\text { activation }\end{array}$ & $\begin{array}{l}\text { I) Impregnation }\left(\mathrm{H}_{3} \mathrm{PO}_{4}, 100{ }^{\circ} \mathrm{C} \text {, }\right. \\
\text { 24h) } \\
\text { II) Carbonization }\left(500{ }^{\circ} \mathrm{C}, 1 \mathrm{~h}\right)\end{array}$ & Tangerine peels & Acetamiprid & $\begin{array}{l}\mathrm{pH}=5.0 \\
\mathrm{C}_{0}=25 \mathrm{mg} \mathrm{L} \\
\mathrm{V}=100 \mathrm{~mL}, \mathrm{~m}=0.1 \mathrm{~g} \\
\mathrm{~T}=308 \mathrm{~K}, \mathrm{t}=240 \mathrm{~min}\end{array}$ & Langmuir & $\begin{array}{l}\text { Pseudo-second- } \\
\text { order }\end{array}$ & 35.7 & Mohammad et al. (2020) \\
\hline $\begin{array}{l}\text { Chemical } \\
\text { activation }\end{array}$ & $\begin{array}{l}\text { I) Impregnation }\left(\mathrm{H}_{3} \mathrm{PO}_{4}, 24 \mathrm{~h}\right) \\
\text { II) Carbonization }\left(700^{\circ} \mathrm{C}, 4 \mathrm{~h}\right)\end{array}$ & Tangerine seed & Bendiocarb & $\begin{array}{l}\mathrm{pH}=7.0 \\
\mathrm{C}_{0}=10 \mathrm{mg} \mathrm{L} \\
\mathrm{V}=10 \mathrm{~mL}, \mathrm{~m}=10 \mathrm{mg} \\
\mathrm{T}=303 \mathrm{~K}, \mathrm{t}=12 \mathrm{~min}\end{array}$ & Langmuir & $\begin{array}{l}\text { Pseudo-second- } \\
\text { order }\end{array}$ & 7.97 & (Y. Wang et al., 2020) \\
\hline $\begin{array}{l}\text { Chemical } \\
\text { activation }\end{array}$ & $\begin{array}{l}\text { I) Impregnation }\left(\mathrm{H}_{3} \mathrm{PO}_{4}, 24 \mathrm{~h}\right) \\
\text { II) Carbonization }\left(700^{\circ} \mathrm{C}, 4 \mathrm{~h}\right)\end{array}$ & Tangerine seed & Metolcarb & $\begin{array}{l}\mathrm{pH}=7.0 \\
\mathrm{C}_{0}=10 \mathrm{mg} \mathrm{L} \\
\mathrm{V}=10 \mathrm{~mL}, \mathrm{~m}=10 \mathrm{mg} \\
\mathrm{T}=303 \mathrm{~K}, \mathrm{t}=12 \mathrm{~min}\end{array}$ & Langmuir & $\begin{array}{l}\text { Pseudo-second- } \\
\text { order }\end{array}$ & 9.11 & (Y. Wang et al., 2020) \\
\hline $\begin{array}{l}\text { Chemical } \\
\text { activation }\end{array}$ & $\begin{array}{l}\text { I) Impregnation }\left(\mathrm{H}_{3} \mathrm{PO}_{4}, 24 \mathrm{~h}\right) \\
\text { II) Carbonization }\left(700^{\circ} \mathrm{C}, 4 \mathrm{~h}\right)\end{array}$ & Tangerine seed & Isoprocarb & $\begin{array}{l}\mathrm{pH}=7.0 \\
\mathrm{C}_{0}=10 \mathrm{mg} \mathrm{L}^{-1} \\
\mathrm{~V}=10 \mathrm{~mL}, \mathrm{~m}=10 \mathrm{mg} \\
\mathrm{T}=303 \mathrm{~K}, \mathrm{t}=12 \mathrm{~min}\end{array}$ & Langmuir & $\begin{array}{l}\text { Pseudo-second- } \\
\text { order }\end{array}$ & 13.95 & (Y. Wang et al., 2020) \\
\hline $\begin{array}{l}\text { Chemical } \\
\text { activation }\end{array}$ & $\begin{array}{l}\text { I) Impregnation }\left(\mathrm{H}_{3} \mathrm{PO}_{4}, 24 \mathrm{~h}\right) \\
\text { II) Carbonization }\left(700^{\circ} \mathrm{C}, 4 \mathrm{~h}\right)\end{array}$ & Tangerine seed & Pirimicarb & $\begin{array}{l}\mathrm{pH}=7.0 \\
\mathrm{C}_{0}=10 \mathrm{mg} \mathrm{L} \\
\mathrm{V}=10 \mathrm{~mL}, \mathrm{~m}=10 \mathrm{mg} \\
\mathrm{T}=303 \mathrm{~K}, \mathrm{t}=12 \mathrm{~min}\end{array}$ & Langmuir & $\begin{array}{l}\text { Pseudo-second- } \\
\text { order }\end{array}$ & 39.37 & (Y. Wang et al., 2020) \\
\hline $\begin{array}{l}\text { Chemical } \\
\text { activation }\end{array}$ & $\begin{array}{l}\text { I) Impregnation }\left(\mathrm{H}_{3} \mathrm{PO}_{4}, 24 \mathrm{~h}\right) \\
\text { II) Carbonization }\left(700^{\circ} \mathrm{C}, 4 \mathrm{~h}\right)\end{array}$ & Tangerine seed & Carbaryl & $\begin{array}{l}\mathrm{pH}=7.0 \\
\mathrm{C}_{0}=10 \mathrm{mg} \mathrm{L} \\
\mathrm{V}=10 \mathrm{~mL}, \mathrm{~m}=10 \mathrm{mg} \\
\mathrm{T}=303 \mathrm{~K}, \mathrm{t}=12 \mathrm{~min}\end{array}$ & Langmuir & $\begin{array}{l}\text { Pseudo-second- } \\
\text { order }\end{array}$ & 44.64 & (Y. Wang et al., 2020) \\
\hline $\begin{array}{l}\text { Chemical } \\
\text { activation }\end{array}$ & $\begin{array}{l}\text { I) Impregnation }\left(\mathrm{H}_{3} \mathrm{PO}_{4}, 24 \mathrm{~h}\right) \\
\text { II) Carbonization }\left(700^{\circ} \mathrm{C}, 4 \mathrm{~h}\right)\end{array}$ & Tangerine seed & Methiocarb & $\begin{array}{l}\mathrm{pH}=7.0 \\
\mathrm{C}_{0}=10 \mathrm{mg} \mathrm{L} \\
\mathrm{V}=10 \mathrm{~mL}, \mathrm{~m}=10 \mathrm{mg} \\
\mathrm{T}=303 \mathrm{~K}, \mathrm{t}=12 \mathrm{~min}\end{array}$ & Langmuir & $\begin{array}{l}\text { Pseudo-second- } \\
\text { order }\end{array}$ & 93.46 & (Y. Wang et al., 2020) \\
\hline $\begin{array}{l}\text { Chemical } \\
\text { activation }\end{array}$ & $\begin{array}{l}\text { I) Impregnation with } \mathrm{ZnCl}_{2} \\
\text { II) Carbonization }\left(500^{\circ} \mathrm{C}, 2 \mathrm{~h}, \mathrm{~N}_{2}\right)\end{array}$ & Orange pulp & 2,4-Dichlorophenoxy acetic acid & $\begin{array}{l}\mathrm{pH}=6.22 \\
\mathrm{C}_{0}=100 \mathrm{mg} \mathrm{L}^{-1} \\
\mathrm{~V}=100 \mathrm{~mL}, \mathrm{~m}=0.3 \mathrm{~g} \\
\mathrm{~T}=298 \mathrm{~K}, \mathrm{t}=300 \mathrm{~min}\end{array}$ & Langmuir & $\begin{array}{l}\text { Pseudo-second- } \\
\text { order }\end{array}$ & 71.94 & Angın and Güneş (2020) \\
\hline $\begin{array}{l}\text { Physical } \\
\text { activation }\end{array}$ & $\begin{array}{l}\text { I) Carbonization }\left(800^{\circ} \mathrm{C}, 1 \mathrm{~h}\right) \\
\left.\text { II) Activation (Steam, } 200^{\circ} \mathrm{C}\right)\end{array}$ & Sugarcane bagasse & 2,4-Dichlorophenoxy acetic acid & $\begin{array}{l}\mathrm{pH}=6.86 \\
\mathrm{C}_{0}=5 \mathrm{mg} \mathrm{L}^{-1} \\
\mathrm{~V}=25 \mathrm{~mL}, \mathrm{~m}=10 \mathrm{mg} \\
\mathrm{T}=300 \mathrm{~K}, \mathrm{t}=48 \mathrm{~h}\end{array}$ & $\begin{array}{l}\text { Redlich-Peterson } \\
\text { Langmuir }\end{array}$ & $\begin{array}{l}\text { Pseudo-second- } \\
\text { order }\end{array}$ & 153.9 & Brito et al. (2020) \\
\hline $\begin{array}{l}\text { Physical } \\
\text { activation }\end{array}$ & $\begin{array}{l}\text { I) Carbonization }\left(800{ }^{\circ} \mathrm{C}, 1 \mathrm{~h}\right) \\
\left.\text { II) Activation (Steam, } 200^{\circ} \mathrm{C}\right)\end{array}$ & Coconut shell & 2,4-Dichlorophenoxy acetic acid & $\begin{array}{l}\mathrm{pH}=6.86 \\
\mathrm{C}_{0}=5 \mathrm{mg} \mathrm{L} \\
\mathrm{V}=25 \mathrm{~mL}, \mathrm{~m}=10 \mathrm{mg} \\
\mathrm{T}=300 \mathrm{~K}, \mathrm{t}=48 \mathrm{~h}\end{array}$ & $\begin{array}{l}\text { Redlich-Peterson } \\
\text { Langmuir }\end{array}$ & $\begin{array}{l}\text { Pseudo-second- } \\
\text { order }\end{array}$ & 233.0 & Brito et al. (2020) \\
\hline $\begin{array}{l}\text { Physical } \\
\text { activation }\end{array}$ & $\begin{array}{l}\text { I) Carbonization }\left(800^{\circ} \mathrm{C}, 1 \mathrm{~h}\right) \\
\left.\text { II) Activation (Steam, } 200^{\circ} \mathrm{C}\right)\end{array}$ & Endocarp of babassu & 2,4-Dichlorophenoxy acetic acid & $\begin{array}{l}\mathrm{pH}=6.86 \\
\mathrm{C}_{0}=5 \mathrm{mg} \mathrm{L}^{-1} \\
\mathrm{~V}=25 \mathrm{~mL}, \mathrm{~m}=10 \mathrm{mg} \\
\mathrm{T}=300 \mathrm{~K}, \mathrm{t}=48 \mathrm{~h}\end{array}$ & $\begin{array}{l}\text { Redlich-Peterson } \\
\text { Langmuir }\end{array}$ & $\begin{array}{l}\text { Pseudo-second- } \\
\text { order }\end{array}$ & 235.5 & Brito et al. (2020) \\
\hline
\end{tabular}


ibuprofen ( $\left.\log \mathrm{K}_{\mathrm{ow}}=3.97\right)$, and triclosan $\left(\log \mathrm{K}_{\mathrm{ow}}=4.76\right)$, have been evaluated for adsorption onto commercial powdered activated carbon from aqueous solutions (Kaur et al., 2018). The removal efficacy and adsorption capacity were established in the order caffeine < ibuprofen < triclosan, which correlates to the octanolwater partition coefficient (log $\mathrm{K}_{\mathrm{ow}}$ ) and the decreased solubility of the micro-pollutants in water. The obtained results established that the lipophilicity of micro-pollutants displays an important role in adsorption onto activated carbons.

The activated carbon fiber has been deployed for the adsorption of non-ionic carbamazepine, anionic diclofenac, and cationic propranolol from aqueous solutions (Zhao et al., 2020). The activated carbon fiber sorption capability was evaluated by the $\mathrm{pH}$, isotherm, kinetic, and ionic strength experiments. The carbamazepine adsorption onto activated carbon fiber was independent of ionic strength and $\mathrm{pH}$, while the anionic diclofenac adsorption was reduced at high concentrations of $\mathrm{NaCl}$ and alkaline $\mathrm{pHs}$. The kinetic studies revealed the fast sorption of cationic propranolol and uncharged carbamazepine, and relatively slow sorption of anionic diclofenac onto activated carbon fiber. Based on the isotherm studies, the calculated $\mathrm{q}_{\max }\left(\mathrm{mmol} \mathrm{g}^{-1}\right)$ of activated carbon fiber for diclofenac $(0.95 \pm 0.08)$, propranolol $(1.07 \pm 0.08)$, and carbamazepine $(1.27 \pm 0.06)$ exhibited the potent ability of activated carbon fiber for removing the pharmaceutical pollutants from real samples.

The activated carbon prepared from Reynoutria japonica invasive herb was reported for the xenobiotics (diclofenac and paracetamol) sorption from water (Fig. 7) (Koutník et al., 2020). The activated carbons were synthesized by microwave treatment using sodium methanolate (MeNa), $\mathrm{NaOH}$, and $\mathrm{H}_{3} \mathrm{PO}_{4}$ chemicals. The type of activating agent was an important factor with a significant effect on the result of sorbents' texture and the sorbent's surface chemical nature. The activated carbon- $\mathrm{H}_{3} \mathrm{PO}_{4}$ displayed the highest sorption capacity for diclofenac $\left(87.09 \mathrm{mg} \mathrm{g}^{-1}\right)$ and paracetamol $\left(136.61 \mathrm{mg} \mathrm{g}^{-1}\right)$. The activating agent selection, the $\pi-\pi$ interactions, and the interactions between the functional groups of activated carbon and sorbate played an important role in the adsorption rate.

The hierarchical porous activated carbon (SCB-AC) with a high specific surface area (1145 $\mathrm{m}^{2} \mathrm{~g}^{-1}$ ) was produced from sugar cane bagasse via a single-step $\mathrm{ZnCl}_{2}$ activation and then applied for the adsorption of diclofenac sodium (DFC) from contaminated aqueous solutions in a batch system (Abo El Naga et al., 2019). The adsorption experiments exhibited that the fast adsorption of DFC onto SCB-AC occurred with an adsorption capacity of $315.0 \mathrm{mg} \mathrm{g}^{-1}$. The SCB-AC not only demonstrated a rapid removal rate and high maximum adsorption capacity for DFC but also displayed high stability and prominent recyclability through simple washing of the DFC-saturated sorbent with acetone. Also, the $\mathrm{pH}$ effect on the sorbent surface charge and DCF sorption was studied for predicting the mechanism. At optimal adsorption $\mathrm{pH} 2, \mathrm{pH}$ value lower than the DFC pKa (4.2) and SCB-AC pH PZC (7.3), the high DFC adsorption was related to non-electrostatic adsorption mechanisms, including hydrogen bonding, hydrophobic, and $\pi-\pi$ electron donor-acceptor (EDA) interactions.

The activated carbon has been synthesized by chemical activation of Tara gum using $\mathrm{FeCl}_{3}$ for antipyrine removal (Fig. 8); synthesis was performed with different ratios of activating agent and precursor $\left(\mathrm{r}=\mathrm{FeCl}_{3} /\right.$ precursor, $0.5-3.0 \mathrm{wt} \%$ ) at variable temperatures from 400 to $1000{ }^{\circ} \mathrm{C}$. The highly porous structure was obtained at $\mathrm{r}=2$ and $800^{\circ} \mathrm{C}$. The kinetic and isotherm studies of the adsorption process exhibited that the experimental adsorption data fitted well with the hyperbolic kinetic and Langmuir isotherm equations. The adsorption results affirmed that the activated carbon derived from Tara gum with $\mathrm{q}_{\max }=307.7\left(\mathrm{mg} \mathrm{g}^{-1}\right)$ was useful for the adsorption of antipyrine (Bedia et al., 2018).

Activated carbon has also been synthesized from pomegranate wood wastes using an innovated $\mathrm{NH}_{4} \mathrm{Cl}$-induced activation process $\left(\mathrm{NH}_{4} \mathrm{Cl}\right.$-modified activated carbon; NAC) and was evaluated for chlortetracycline adsorption from aqueous solutions (Fig. 9) (Alahabadi et al., 2017). The adsorption ability of NAC was compared with standard activated carbon (SAC) for chlortetracycline adsorption. The NAC comprised a mesoporous granular particle with higher functional groups, greater surface area, and larger pore volume with much greater chlortetracycline removal and adsorption capacity compared to SAC under similar optimum conditions. The $\mathrm{NH}_{4} \mathrm{Cl}$-induced chemical treatment generated more crystallinity with highly ordered pores for NAC, probably due to the $\mathrm{NH}_{4} \mathrm{Cl}$ explosive characteristics. The studied isotherm and kinetic models exhibited more favourability of chlortetracycline sorption onto NAC through the physical $\pi-\pi$ EDA interaction.

Paredes-Laverde et al. prepared activated carbons $\left(\mathrm{CH}-\mathrm{ZnCl}_{2}\right.$ activated carbon and $\mathrm{RH}-\mathrm{NaOH}$ activated carbon) from coffee husk $(\mathrm{CH})$ and rice husk $(\mathrm{RH})$, via impregnation with activating agents $\mathrm{ZnCl}_{2}$ and $\mathrm{NaOH}$ (Fig. 10) (Paredes-Laverde et al., 2019). The synthesized activated carbons were evaluated for acetaminophen removal from both the distilled water and synthetic urine. The RH$\mathrm{NaOH}$ activated carbon with high carbon content displayed the best results in both media due to the creation of micro-porosity in the activation process and higher surface area. The evaluation of temperature influence in the range of $500-800{ }^{\circ} \mathrm{C}$ on the preparation of $\mathrm{RH}-\mathrm{NaOH}$ activated carbons ( $\mathrm{RH}-\mathrm{NaOH}-500, \mathrm{RH}-\mathrm{NaOH}-650$, and $\mathrm{RH}-\mathrm{NaOH}-800$ ) revealed that the functional groups and elemental composition of activated carbons were altered with temperature changes. Furthermore, the rise in temperature during the activation process helped to increase the porosity and surface area. Thus, the $\mathrm{RH}-\mathrm{NaOH}-800$ activated carbon showed the highest adsorption efficiency and removed the acetaminophen from aqueous solutions via hydrogen bonding and $\pi-\pi$ interactions. Table 2 lists some of the reported papers regarding drug adsorption by activated carbons.

4.2.1.2. Pesticides adsorption onto activated carbons. Many researchers have investigated the elimination of pesticides from aqueous solutions using activated carbons, e.g., metaldehyde (Rolph et al., 2018), paclobutrazol (Grant et al., 2018), 11 pesticides (Grant et al., 2019), iodosulfuron (Ahmad, 2019), carbendazim and linuron (Hgeig et al., 2019), carbendazim (T. Wang et al., 2019), and 2,4-dichlorophenoxyacetic acid (2,4-D) (Amiri et al., 2020).

Two commercial activated carbons (GAB; $\mathrm{S}_{\mathrm{Mic}}=580 \mathrm{~m}^{2} \mathrm{~g}^{-1}$, $\mathrm{V}_{\text {Mic }}=0.27 \mathrm{~cm}^{3} \mathrm{~g}^{-1}, \mathrm{pH}_{\mathrm{PZC}}=7.46$ and CBP; $\mathrm{S}_{\mathrm{Mic}}=99 \mathrm{~m}^{2} \mathrm{~g}^{-1}$, $\mathrm{V}_{\text {Mic }}=0.04 \mathrm{~cm}^{3} \mathrm{~g}^{-1}, \mathrm{pH}_{\mathrm{PZC}}=4.76$ ) were utilized for the adsorption of 4-chloro-2-methylphenoxyacetic acid (MCPA; pKa = 3.07) and 2,4-dichlorophenoxyacetic acid $(2,4-\mathrm{D} ; \mathrm{pKa}=2.73)$ from aqueous solutions (Spaltro et al., 2018). The microporous GAB exhibited higher adsorption capacity $\left(\mathrm{q}_{\max }=367.15 \mathrm{mg} \mathrm{g}^{-1}\right.$ ) in comparison to CBP $\left(\mathrm{q}_{\max }=273.07 \mathrm{mg} \mathrm{g}^{-1}\right)$. The results of adsorption evaluation demonstrated that ionic strength and $\mathrm{pH}$ played a decisive role in the adsorption rate, which was enhanced for both the pesticides by increasing the ionic strength. According to the $\mathrm{pH}_{\mathrm{PzC}}$ of activated carbons and pesticides $\mathrm{pKa}$, the MCPA and 2,4-D removal were reduced with increasing of $\mathrm{pH}(\mathrm{pH}>4.76)$. The obtained adsorption data declare that electrostatic interaction between the activated carbons surface and pesticides were governing the adsorption mechanism.

The adsorption of diazinon from the aqueous solution was investigated using batch and fixed-bed column techniques onto a walnut shell activated carbon modified by impregnation with $\mathrm{H}_{3} \mathrm{PO}_{4}$ (WSAC; $\mathrm{S}_{\mathrm{BET}}=1434 \mathrm{~m}^{2} \mathrm{~g}^{-1}$ ) (Bayat et al., 2018). The adsorption results in the batch technique confirmed that the 

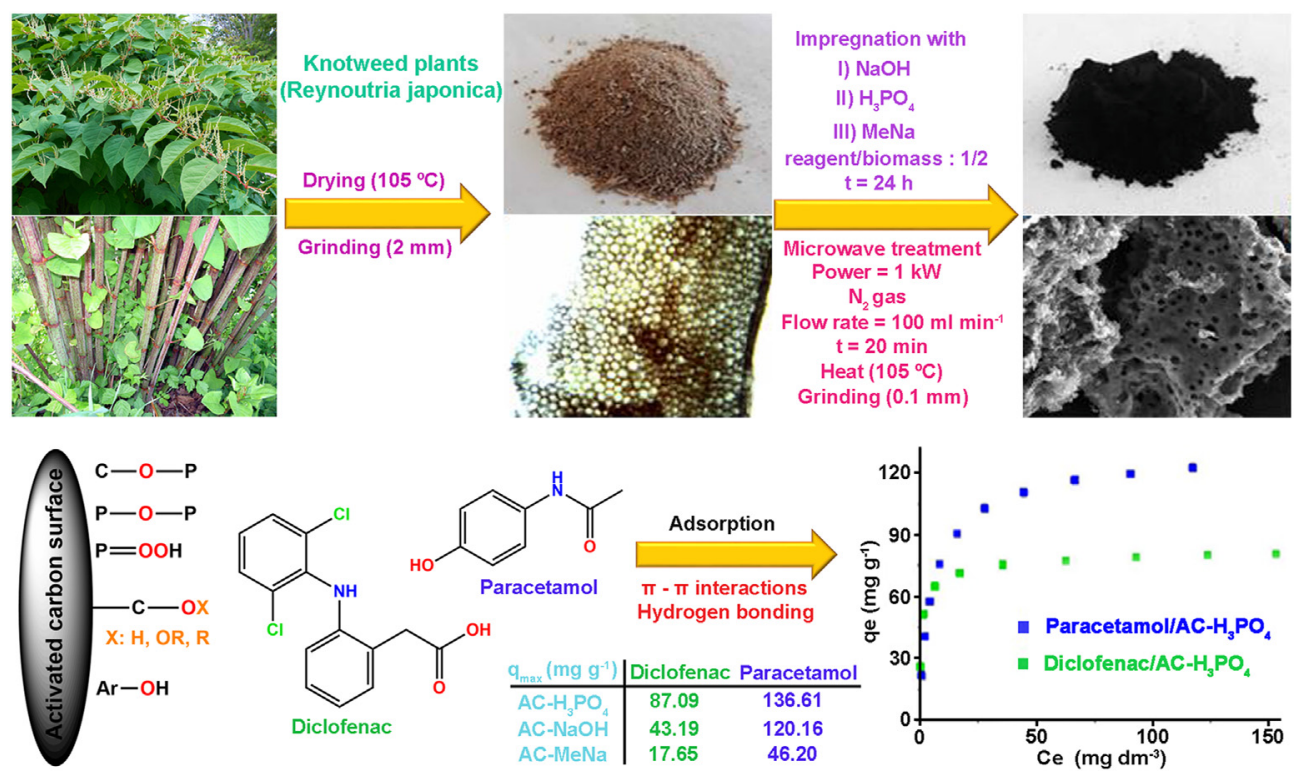

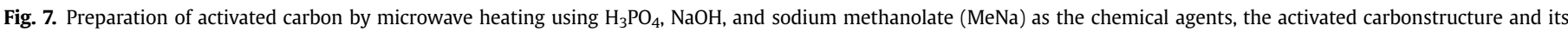

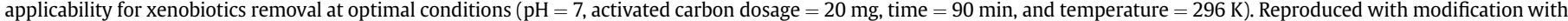
permission from (Koutník et al., (2020)).
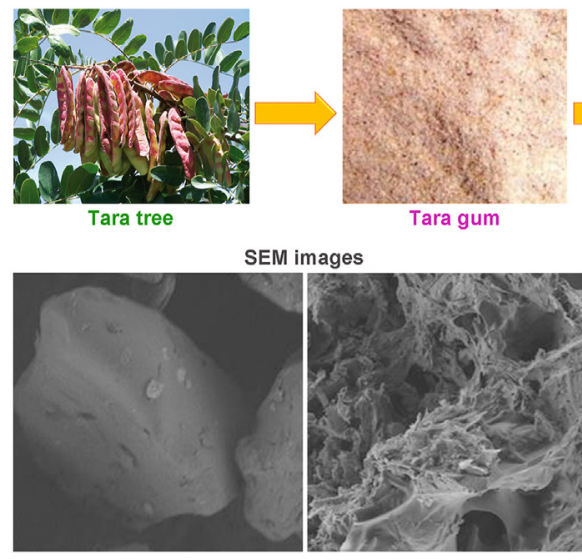

Tara gum

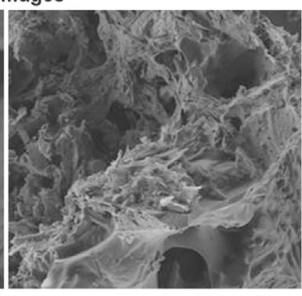

GT2-800 Activated carbon GT2-800 $\left(r=2, T=800^{\circ} \mathrm{C}\right)$

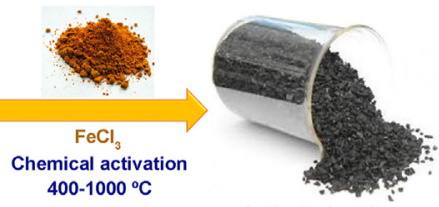

$400-1000^{\circ} \mathrm{C}$

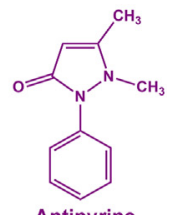

Adsorption

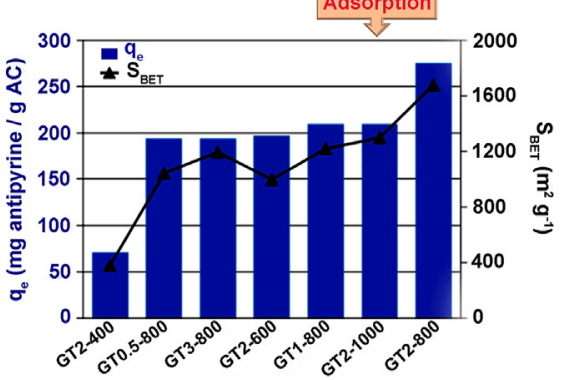

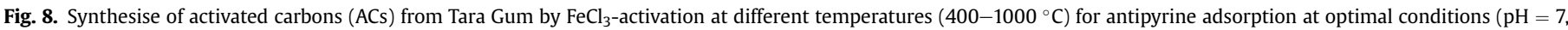

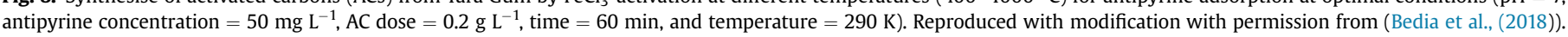

pseudo-second-order kinetic and the Freundlich isotherm were the best models to analyze the experimental adsorption data. The adsorption study, due to the high adsorption capacity of WSAC in batch performance $\left(\mathrm{q}_{\max }=169.49 \mathrm{mg} \mathrm{g}^{-1}\right)$, was carried out via a fixed-bed column for compliance with the industrial scale. In the continuous fixed-bed study, the flow rate and bed length were the effective experimental parameters on the adsorption efficiency; bed $\mathrm{q}_{\max }$ of $34.98 \mathrm{mg} \mathrm{g}^{-1}$ was achieved with flow rates $=12 \mathrm{~L} \mathrm{~h}^{-1}$ and bed length $=30 \mathrm{~cm}$.

Sou et al. reported the starch-derived mesoporous activated carbon that was used for the elimination of 11 pesticides from aqueous solutions (Suo et al., 2019). The examined pesticides comprised atrazine, thiacloprid, pymetrozine, difenoconazole, imazalil, azoxystrobin, pyraclostrobin, trifloxystrobin, chlorantraniliprole, acetamiprid, and diuron. The adsorption results displayed that the starch-derived AC removed the pesticides very fast (5 min) and with good adsorption efficiency (atrazine $=84 \%$, thiacloprid $=96 \%$, pymetrozine $=99 \%$, difenoconazole $=92 \%$, imazalil $=95 \%$, azoxystrobin $=90 \%$, pyraclostrobin $=94 \%$, trifloxystrobin $=85 \%$, chlorantraniliprole $=82 \%$, acetamiprid $=96 \%$ and diuron $=96 \%$ ). The evaluation of the adsorption mechanism validated that oxygen or nitrogen-containing functional groups and $\pi$-bonding networks of benzene in the AC structure promoted pesticide adsorption.

The orange peel activated carbon (OPAC) was prepared from orange peel waste for removal of chlorophenoxyacetic acid pesticides (2,4-dichlorophenoxyacetic acid; 2,4-D, 2-(2,4dichlorophenoxy)propionic acid; 2,4-DP, 2-methyl-4chlorophenoxyacetic acid; MCPA, methylchlorophenoxypropionic acid; MCPP and 2,4,5-trichlorophenoxyacetic acid; 2,4,5-T) from aqueous solutions (Fig. 11a) (Pandiarajan et al., 2018). The OPAC with high surface area $\left(592.471 \mathrm{~m}^{2} \mathrm{~g}^{-1}\right)$ exhibited good $\mathrm{q}_{\max }$ for the adsorption of chlorophenoxyacetic acid pesticides (2,4-D: $\mathrm{q}_{\max }=515.46,2,4-\mathrm{DP}: \mathrm{q}_{\max }=215.52, \mathrm{MCPA}: \mathrm{q}_{\max }=414.94,2,4,5-\mathrm{T}$ : 


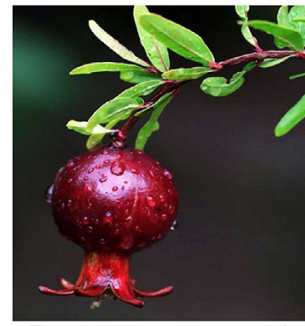

Pomegranate wood
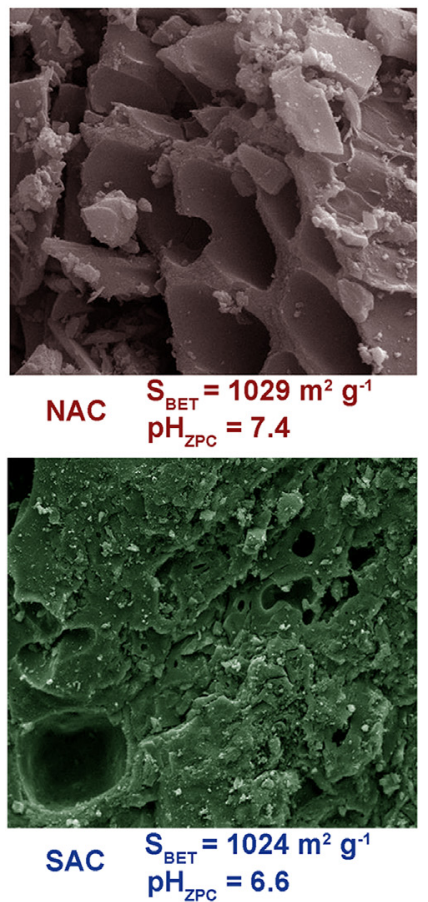

I) Carbonization

$\left(700^{\circ} \mathrm{C}, 1 \mathrm{~h}, \mathrm{~N}_{2}\right)$

II) $\mathrm{NH}_{4} \mathrm{Cl}$

(2.0 $\left.0^{4} \%, 105^{\circ} \mathrm{C}, 24 \mathrm{~h}\right)$

III) Pyrolysis

$\left(800^{\circ} \mathrm{C}, 2.5 \mathrm{~h}, \mathrm{~N}_{2}\right)$

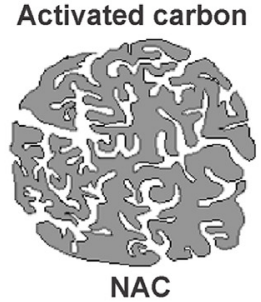

(With -OH and - $\mathrm{COOH}$ groups)

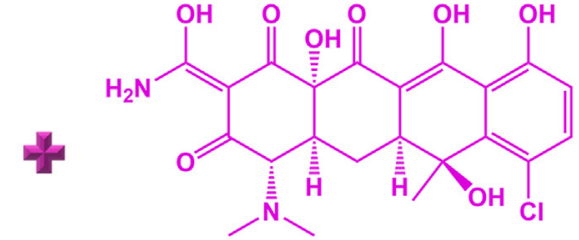

Chlortetracycline (CTCN; pKa = 4.5)

Adsorption

Adsorption mechanism

At low pH: $\mathrm{ACs}^{+} \mathrm{CTCN}^{+}$

Electrostatic repulsion

At very high $\mathrm{pH}$ : ACs- $\mathrm{CTCN}^{-}$ Electrostatic repulsion

At $\mathrm{pH}=6\left(\mathrm{pH}<\mathrm{pH}_{\mathrm{zPC}}\right)$

$\mathrm{ACs}^{+}$Zwitterions CTCN

Physical $\pi-\pi$ EDA interaction

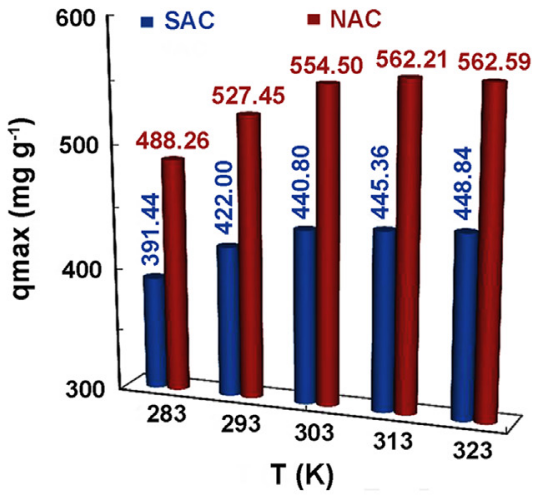

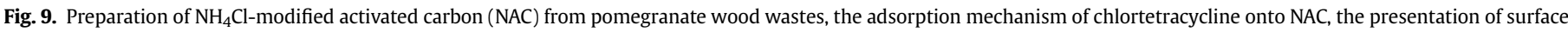

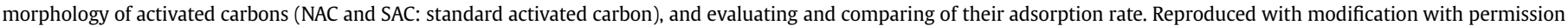
from (Alahabadi et al., (2017)).

$\mathrm{q}_{\max }=416.67$, and MCPP: $\left.\mathrm{q}_{\max }=574.71 \mathrm{mg} \mathrm{g}^{-1}\right)$. The investigation of isotherm and kinetic models proved that the experimental adsorption results fitted well with the Langmuir isotherm and pseudo-second-order kinetic. Besides, the obtained mean sorption energy $\left(E<8 \mathrm{~kJ} \mathrm{~mol}^{-1}\right.$ ) from Dubinin-Radushkevich isotherm suggested that the physical sorption occurred in the adsorption process.

The application of orange (Citrus sinensis L.) pulp activated carbon (OPAC) was also reported for the elimination of 2,4-D from aqueous media (Angın and Güneş, 2020). The adsorption results showed that OPAC with $\mathrm{S}_{\mathrm{BET}}=1779\left(\mathrm{~m}^{2} \mathrm{~g}^{-1}\right)$ and $\mathrm{q}_{\max }=71.94(\mathrm{mg}$ $\mathrm{g}^{-1}$ ) displayed good ability for the elimination of 2,4-D pesticide.

The tangerine seed activated carbons (TSAC1-9) were prepared via a one-step pyrolysis method and used for the removal of carbamate pesticides (bendiocarb, metolcarb, isoprocarb, pirimicarb, carbaryl, and methiocarb) from complex solutions (Fig. 11b) (Y. Wang et al., 2020). The evaluation of carbonization temperature and time effects on adsorption efficiency showed that TSAC8 (carbonization temperature $=600{ }^{\circ} \mathrm{C}$ and carbonization time $=4 \mathrm{~h})$ with a high specific surface area $\left(\mathrm{S}_{\mathrm{BET}}=659.62 \mathrm{~m}^{2} \mathrm{~g}^{-1}\right)$ and large pore volume $\left(0.6203 \mathrm{cc} \mathrm{g}^{-1}\right)$ displayed the fast adsorption rate. The isotherm, kinetic and thermodynamic studies exhibited that the Langmuir and pseudo-second-order models were fitted best with the experimental results, and spontaneous and exothermic carbamate adsorption occurred onto TSAC.

An innovative approach was reported for the diuron adsorption onto different types of activated carbon fiber felt (ACFF; ACFF$\mathrm{CO}_{2}(250)$, ACFF- $\mathrm{CO}_{2}(200)$, and ACFF- $\mathrm{CO}_{2}(200) \mathrm{H}_{2} \mathrm{O}$ ) supported on the microfiltration membrane in a gravitational filtration system (de Camargo Lima Beluci et al., 2021). Tang et al. reported the removal of trace drugs and pesticides (TDPs; pharmaceuticals: trimethoprim, sulfamethoxazole, roxithromycin, mefenamic acid, ibuprofen, diphenhydramine hydrochloride, clarithromycin, carbamazepine, and diclofenac acid, and pesticides: thiamethoxam, prometryn, prochloraz, imidacloprid, dipterex, dimethomorph, difenoconazole, chlorpyrifos, carbendazim, azoxystrobin, atrazine, and acetamiprid) from secondary effluent by four types of typical granular activated carbon (GAC, coal-based GAC, shell GAC, coconut shell GAC, and wooden GAC) (Tang et al., 2020). The shell GAC with multiple functional group contents (Boehm acidic groups $=900$

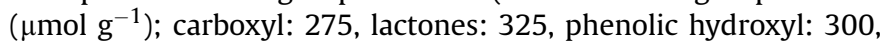
and total Boehm basic groups $=720 \mu \mathrm{mol} \mathrm{g}^{-1}$ ), high surface area $\left(\mathrm{S}_{\mathrm{BET}}=399.06 \mathrm{~m}^{2} \mathrm{~g}^{-1}\right)$ and good adsorption capacity showed a promising efficiency for removing of TDPs via physical and chemical interactions. The recently reported literature on the pesticide adsorption onto activated carbons are presented in Table 2 . 

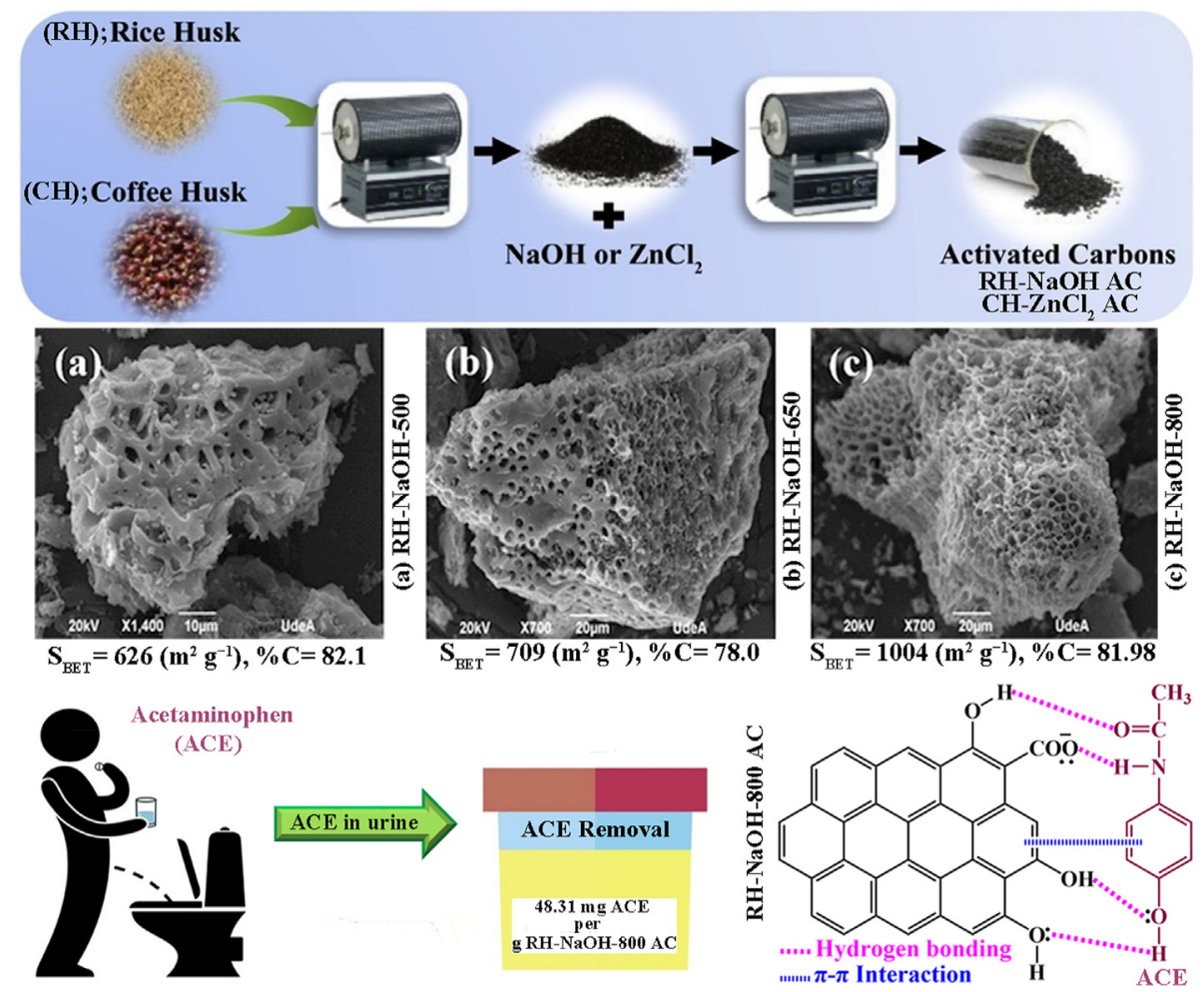

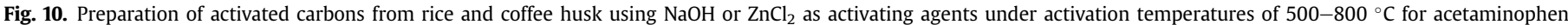

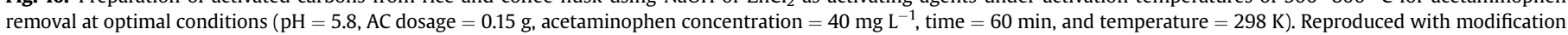
with permission from (Paredes-Laverde et al., (2019)).

\subsubsection{Graphene-based materials}

Graphene is a two-dimensional (2D) crystal made of a monolayer of $\mathrm{sp}^{2}$ hybridized carbon atoms placed in a hexagonal package that was theoretically predicted in 1947 (Wallace, 1947) but prepared in 2004 (Novoselov et al., 2004). It is a building block of many other carbonic materials such as 3D graphite, carbon nanotubes, and Buckyball's, or fullerenes (Fig. 12a) and is prepared from graphite via a mechanical cleavage, to generate the different forms of graphene, namely pristine graphene, graphene oxide (GO), and reduced graphene oxide (Shanmugham et al., 2020). The graphene oxide has a 2D structure as graphene that is created during chemical exfoliation of graphite flakes or oxidation of graphene (Fig. 12b). In the graphene oxide structure, a single sheet of carbon atoms on the basal plane, and the sides are covalently modified with oxygen-containing functional groups (carbonyl, carboxyl, epoxide, or hydroxyl) (Obodo et al., 2019). The reduced graphene oxide is a graphene oxide in which its oxygen contents are reduced by chemical, electrochemical, or thermal reduction (Fig. 12b). The reduction method is developed to distort the honeycomb hexagonal lattice of graphene oxide and also to improve its electrical conductivity (Tarcan et al., 2020). The pristine graphene with an ultra-high specific surface area, but no porosity, naturally repels water. So, the creation of narrow pores and the production of porous graphene allows rapid water permeation (Vijaya Bhaskar Reddy et al., 2019). The porous graphene sheets have been investigated for water filtration due to their capability to prevent the passage of pollutants but only allowing water to permeate through the filter (Ersan et al., 2017). The convenient properties of porous graphene help to prepare lightweight, eco-friendly, and energyefficient water filters (Vijaya Bhaskar Reddy et al., 2019). The graphene oxides with hydrophilic functional groups are well dispersed in water and are good adsorbents in water remediation
(Shanmugham et al., 2020). Thus, graphene and its derivatives with various applications (Yusuf et al., 2019) are attractive adsorbents for the removal of inorganic and organic (Yusuf et al., 2017, 2016, 2015) pollutants from water because of their tunable properties, high surface area, and delocalized $\pi$-electron framework.

4.2.2.1. Adsorption of drugs onto graphene-based materials. Graphene is considered an ideal adsorbent for water remediation because of its high surface area and hydrophobic property that adsorbs the organic pollutants with a strong affinity.

The adsorption of 2-arylpropionic acids (carprofen, ibuprofen, indoprofen, ketoprofen, and naproxen) has been investigated via experimental and theoretical studies (Z. Wang et al., 2019).

The pH-dependent experiments exposed that the 2arylpropionic acids adsorption onto graphene was considerably controlled by the solvent-solute interactions, besides the interactions between sorbate and adsorbent surface. Moreover, the incorporation of molecular docking study with equilibrium adsorption experiments revealed that the interactive strength of 2arylpropionic acids with graphene was significantly correlated to the aromatic moieties rather than the hydrophobicity of sorbate molecules. Results showed that the dominant interaction for the 2arylpropionic acids adsorption onto graphene was $\pi-\pi$ stacking between the graphene bulk $\pi$-system and 2-arylpropionic acids aromatic moieties. The binding energy of adsorbed 2-arylpropionic acids onto graphene followed the order: carprofen $<$ indoprofen $<$ naproxen $<$ ketoprofen $<$ ibuprofen.

The GO revealed higher adsorption capacity and increased adsorptive affinity towards the polar contaminants rather than the graphene due to the presence of polar groups, hydrophilic nature, and good dispersion in water. The GO adsorption rate is related to the degree of oxidation so that highly oxidized GO displayed a 

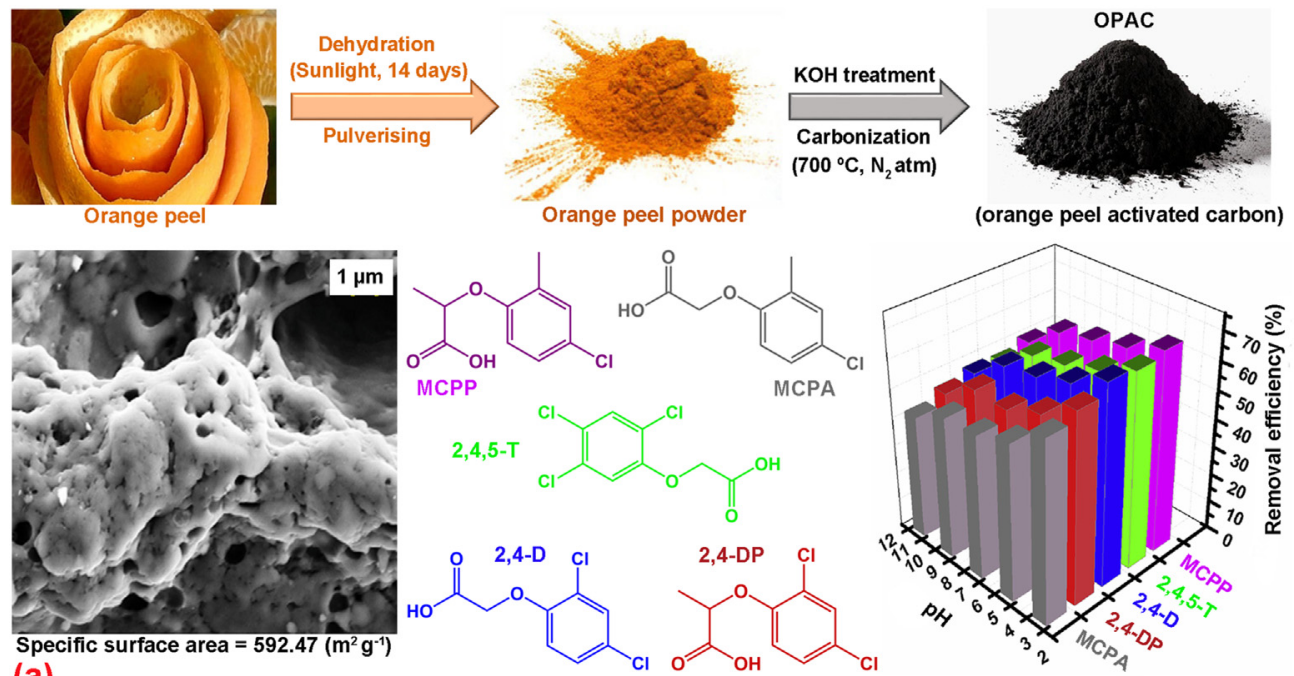

(orange peel activated carbon)

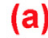

(b)
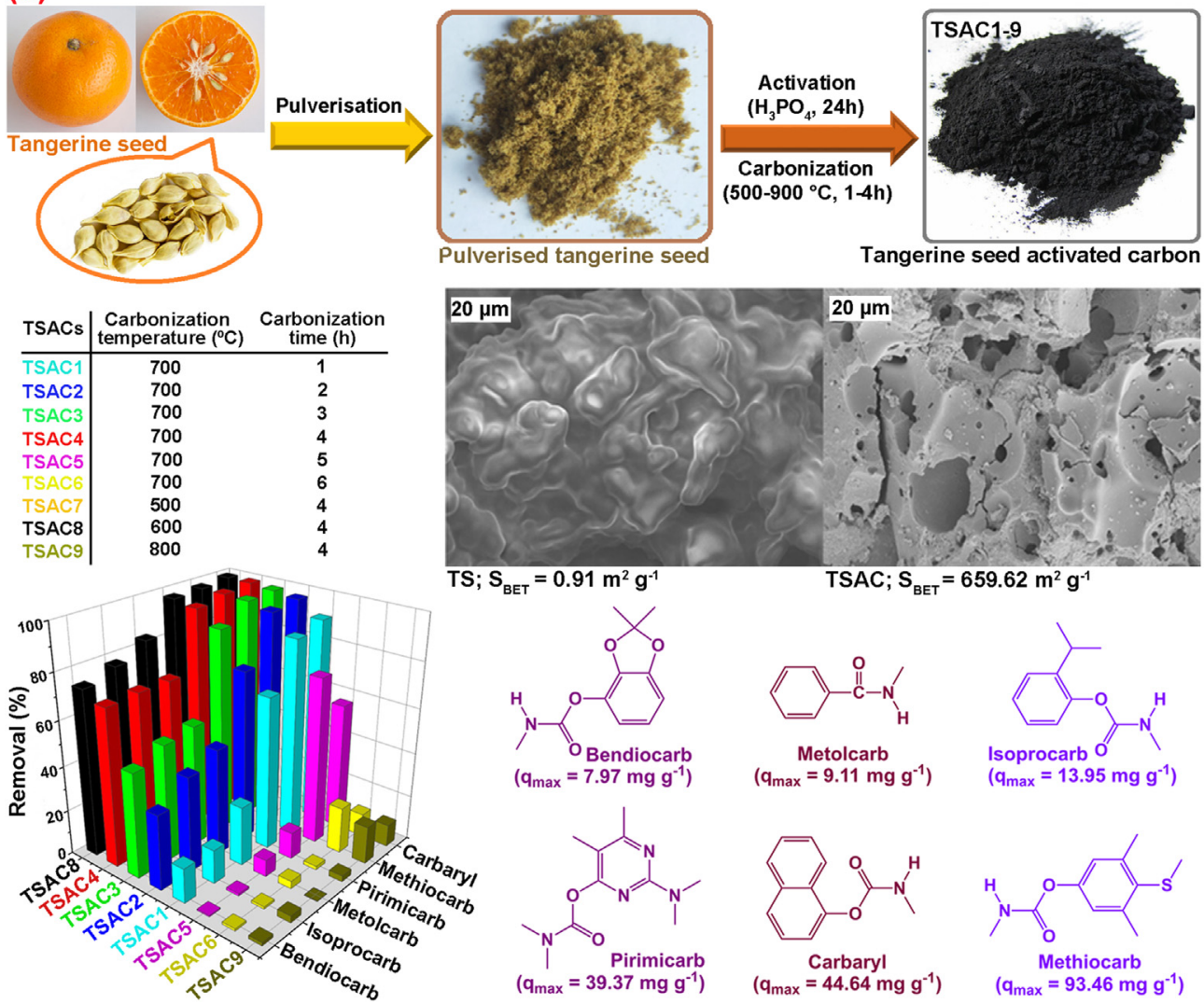

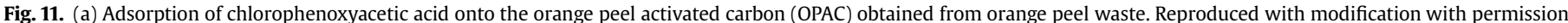

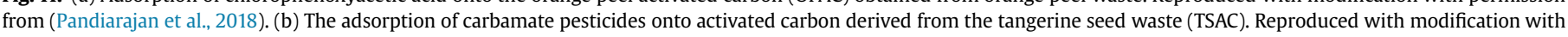
permission from (Y. Wang et al., 2020). (For interpretation of the references to colour in this figure legend, the reader is referred to the Web version of this article.)

superior adsorption rate than the GO with a lower oxidation degree (Thangavel and Venugopal, 2014). In recent years, many research investigations have been conducted for the removal of drugs using GO, for example, adsorption of trimethoprim and isoniazid antibiotics (Çalışkan Salihi et al., 2020), norfloxacin (Moreira et al., 2020), naproxen (Ciğeroğlu et al., 2020), cephalexin (Wernke et al., 2020), sodium diclofenac (Guerra et al., 2019), and fluoroquinolones (ciprofloxacin, norfloxacin, and ofloxacin) (Yadav et al., 2018) from aqueous solutions.

The graphene oxide nanosheet (GOS) has been deployed for the removal of ciprofloxacin, doxycycline, and tetracycline from aqueous streams (Rostamian and Behnejad, 2018). A comprehensive adsorption evaluation of antibiotics onto GOS revealed that the sequence of $\mathrm{q}_{\max }$ was in the following order: tetracycline < doxycycline « ciprofloxacin. The mechanism for the adsorption of ciprofloxacin, doxycycline, and tetracycline onto GOS was assumed to occur via cation $-\pi$ bonding and $\pi-\pi$ interaction, which occurred between the ring moiety of antibiotics and the hexagonal cells of GOS. While, the functional groups of antibiotics interacted with GOS electron-rich surfaces via cation $-\pi$ bonding. 


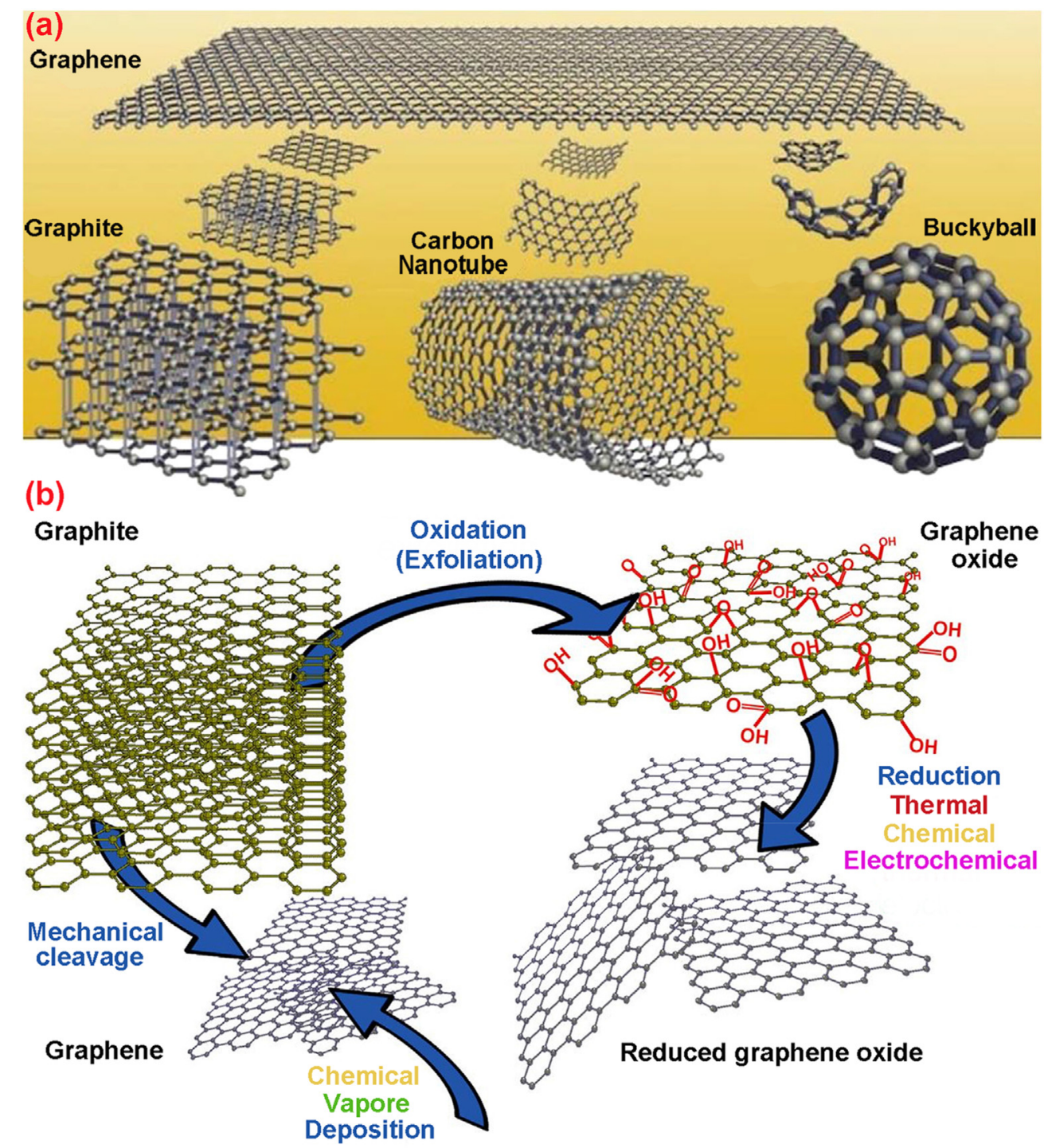

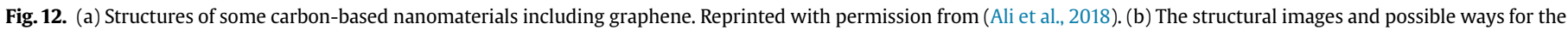
preparation of graphene oxide and reduced graphene oxide. Reprinted with permission from (Selvaraj et al., (2020)).

Although GO showed good adsorption ability for organic pollutants, its collection from the solution is relatively difficult due to its aggregation tendency because of the strong van der Waals force between single layers and its hydrophilicity due to the excess oxygen-containing groups. So, the covalent or non-covalent modification of the GO surface has recently been used to address the dispersibility and aggregation issues (Lawal et al., 2020; Shan et al., 2017).

The porous graphene oxide (PGO) has been synthesized by linking the decafluorobiphenyl (DFB) with GO sheets via a nucleophilic substitution reaction (Fig. 13a) (Shan et al., 2017) where the GO hydroxyl groups reacted with fluorine atoms of DFB to fabricate the PGO adsorbent with enlarged pore size and surface area. The GO-DFB not only displayed an improved sorption capability in comparison to GO and 3D-reduced graphene oxide, but also could be easily separated from the solution. The adsorption efficacy of GO-DFB was evaluated for the removal of sulfamethoxazole, sulfadiazine, phenacetin, paracetamol, ibuprofen, and carbamazepine from aqueous solutions. The adsorption results revealed that GODFB adsorbent was desirable for typical pharmaceuticals adsorption with $\mathrm{q}_{\max }$ carbamazepine $=340.5, \mathrm{q}_{\max }$ sulfamethoxazole $=428.3, q_{\max }$ sulfadiazine $=214.7, \mathrm{q}_{\max }$ ibuprofen $=224.3, q_{\max }$ paracetamol $=350.6$ and $\mathrm{q}_{\max }$ phenacetin $=316.1 \mu \mathrm{mol} \mathrm{g}{ }^{-1}$. The chemical intercalating of rigid molecules proved to be a suitable method to achieve the hydrophilic and layer-stacked PGO for the effective adsorption of pharmaceuticals.

The sulfamethoxazole adsorption onto graphene oxide modified with imidazolium-based ionic liquid (GO-IL) has been reported by Ogunleye et al. (2020). The GO was prepared via the Tour method, then GO-IL was synthesized by co-precipitation of 1-tetradecyl,3butylimidazolium ionic liquid (IL) and GO. The adsorption efficiency of GO-IL was much improved in comparison with GO due to the presence of IL long-chain carbon tail on the GO-IL surface, which increased the hydrophobicity of GO-IL and enhanced its dispersibility in aqueous solutions. The mechanism of sulfamethoxazole sorption onto GO-IL was attributed to hydrophobic effects, hydrogen bonding, and electrostatic- and $\pi-\pi$ interactions.

The GO and non-covalent GO functionalized ionic liquid (IL: 1hexyl,3-decahexyl imidazolium) were prepared and were applied for the elimination of carbamazepine, ketoprofen, and sulfamethoxazole from aqueous media (Fig. 13b) (Lawal et al., 2020). The 
adsorption capacity and adsorption mechanism were determined through theoretical, isotherm, and kinetic studies. The ketoprofen and sulfamethoxazole adsorption onto both graphene oxide and graphene oxide modified with 1-hexyl,3-decahexyl imidazolium was significantly controlled by $\mathrm{pH}$, while the carbamazepine adsorption was not considerably affected by $\mathrm{pH}$ variation. The graphene oxide modified with 1-hexyl,3-decahexyl imidazolium displayed superior adsorption efficacy compared to GO for the removal of carbamazepine, ketoprofen, and sulfamethoxazole. The obtained results revealed that the IL could be favorably used in GO functionalizing to improve its adsorption capacity.

4.2.2.2. Adsorption of pesticides onto graphene-based materials. The effect of graphene-based structures on the elimination of chlorpyrifos and dimethoate from the water has been investigated by Lazarević-Pašti et al. (2018). The obtained results showed that pesticide adsorption onto graphene-based-adsorbents was extremely dependent on the sorbent and sorbate structural properties, and the surface area was not the principal factor controlling the removal efficiency. The aliphatic dimethoate was preferably adsorbed onto hydrophilic oxidized graphene surfaces, whereas the graphene basal plane, containing the $\pi$ electron system and high structural order, removed the chlorpyrifos selectively with aromatic moiety. The intermediate concentration of oxygen functional groups on the surface of graphene-based adsorbent led to the elimination of both the chlorpyrifos and dimethoate.

Recently, the employment of GO as an adsorbent was investigated for the removal of toxic pesticides such as atrazine (de Souza Antônio et al., 2021), ametryn (Khoshnam et al., 2019), chlorpyrifos, and malathion (Yadav et al., 2019) from water and wastewater media.

The GO nanoplatelets were applied for the adsorption of chloridazon and its degradation metabolites (desphenyl-chloridazon: DC and methyl-desphenylchloridazon: MDC) from aqueous solutions (Yan et al., 2020). The good adsorption capacity of GO for chloridazon (67.18 $\mathrm{mg} \mathrm{g}^{-1}$ ), DC (34.30 $\mathrm{mg} \mathrm{g}^{-1}$ ), and MDC (36.85 $\mathrm{mg} \mathrm{g}^{-1}$ ) was established for efficient remediation via hydrogen bonding and hydrophobic- and $\pi-\pi$ interactions.

In addition to adsorption experiments, theoretical insights into the performance of graphene-based materials for the adsorption of pesticides have been explored. The molecular adsorption mechanisms for carbaryl, catechol, and fluridone onto GO were evaluated by Wang et al. (H. Wang et al., 2020). The DFT calculation, molecular dynamics simulation, and binding free energy calculation confirmed that the van der Waals forces and $\pi-\pi$ stacking accounted for the major adsorption interactions between the pesticides and GO.

The ability of graphene and its defected forms, hexagonal boron nitride and carbon doped hexagonal boron nitride, have been explored for the adsorption of atrazine through DFT calculation (Goli et al., 2020). The molecular orbital interactions and energy calculations were assayed to reveal the affinity of graphene, hexagonal boron nitride, and carbon doped hexagonal boron nitride towards atrazine and to estimate the magnitude of the interaction.
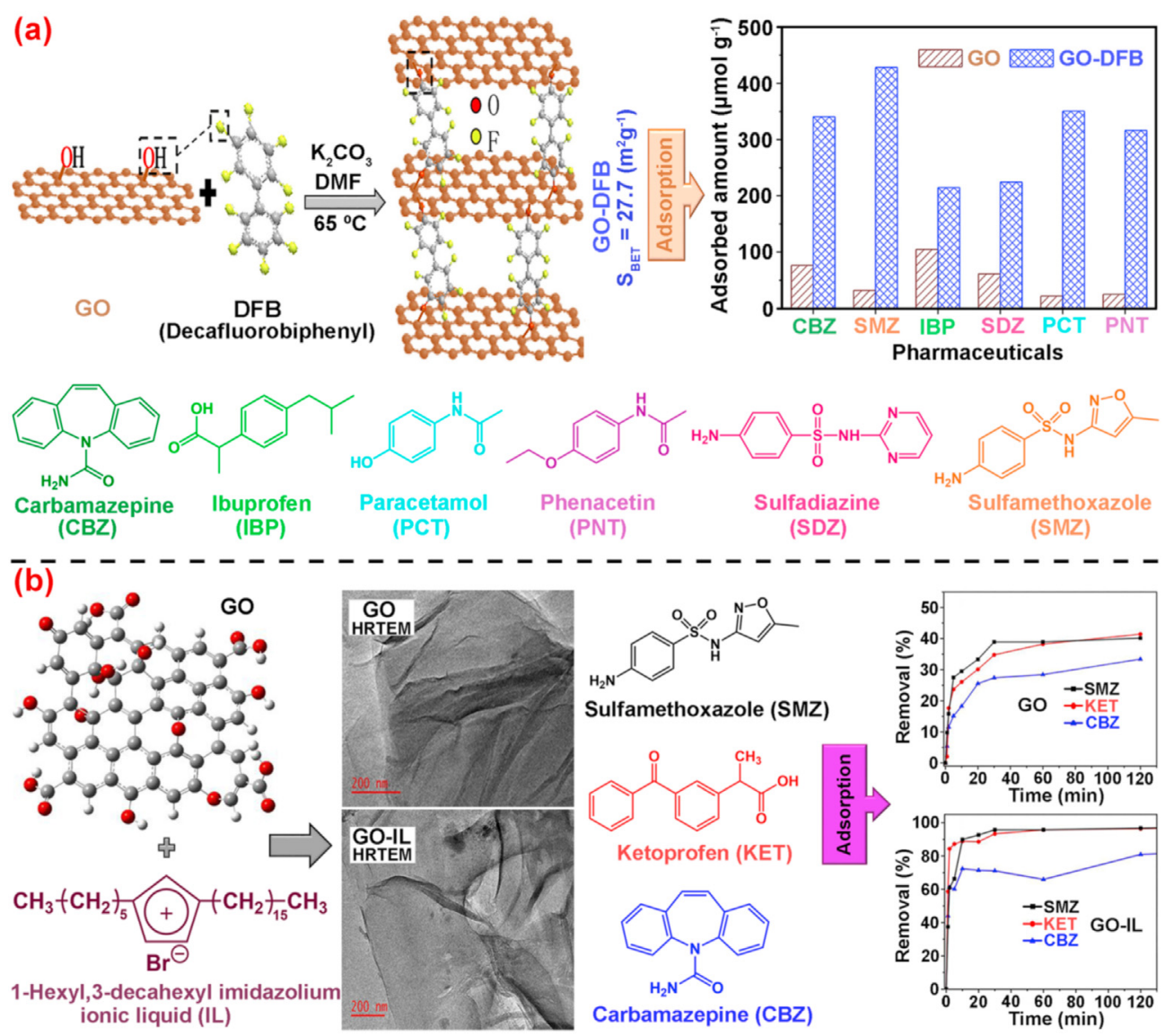

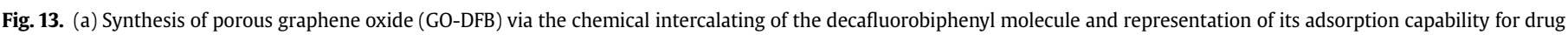

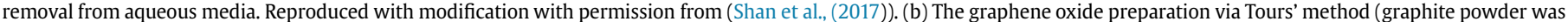

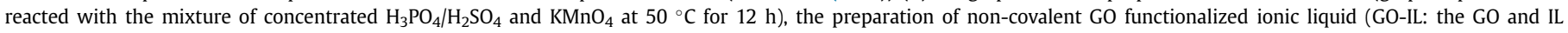

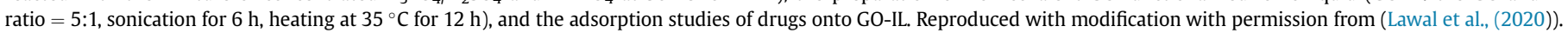


The obtained results indicated that the physical interactions occurred between the atrazine and adsorbents, and the principal role for stabilizing the adsorbed atrazine was related to the dispersive interactions. The mobility of adsorbed atrazine onto graphene adsorbent was affected by the relatively low rotational and translational energy barriers. Whereas, in hexagonal boron nitride, the atrazine movement was limited by electrostatic attractions and polar bonds due to the high translational energy barrier. Table 3 summarizes the adsorption of drugs and pesticides onto graphene-based adsorbents from aqueous solutions.

\subsubsection{Carbon nanotubes}

Carbon nanotubes (CNTs), one-dimensional allotropes of carbon, are the ordered, hollow, and enrolled cylindrical graphenebased materials, comprising $\mathrm{sp}^{2}$-hybridized carbon atoms at the corners of the hexagon. The CNTs can be categorized into two types: I) single-walled CNTs (SWCNTs) that contain a single carbon sheet rolled into a tubular structure, and II) multi-walled CNTs (MWCNTs) that consist of concentric nested SWCNTs with a mutual longitudinal axis (Rawtani et al., 2018). The CNTs have nanometer-sized diameters (SWCNTs $<2 \mathrm{~nm}$ and $2<$ MWCNTs $<100 \mathrm{~nm}$ ), micrometer-sized lengths and length-to-diameter ratio $>1000$ (Sarkar et al., 2018). The SWCNTs were first reported in 1952 by Radushkevich et al. (Radushkevich and Lukyanovich, 1952), and the preparation of multi-walled carbon nanotubes was described by Iijima in 1991 (Iijima, 1991). The employed techniques for the preparation of CNTs preparation include laser ablation, arc discharge, pyrolysis, chemical vapor deposition, and plasmaenhanced chemical vapor deposition (G. Rahman et al., 2019). The potential sites in CNTs responsible for the adsorption of organic micro-pollutants are I) internal sites, II) interstitial channels, III) grooves and IV) the outside surface (Jung et al., 2015). In recent years, CNTs have garnered immense interest to remove organic micro-pollutants from aqueous media. The CNTs, due to their hollow and layered structure, high surface area, small pore size, unique chemical structure, and chemical/thermal stability, serve as excellent sorbents for organic micro-pollutants adsorption (Ahmad et al., 2019). An overview of the adsorption-related information for drugs and pesticides onto CNTs from aqueous solutions is presented in this section.

4.2.3.1. Adsorption of drugs onto carbon nanotubes. The CNTs have been deployed to eliminate a wide range of drugs from water because of their excellent adsorption efficiency; commercial MWCNT was applied for the adsorption of caffeine and diclofenac from aqueous media (Gil et al., 2018). The effect of adsorption parameters such as the drug initial concentration $\left(C_{0}\right)$, MWCNT dosage $(\mathrm{m})$, contact time $(\mathrm{t})$, and temperature $(\mathrm{T})$, has been evaluated for the adsorption behavior of MWCNT. The obtained results showed that the maximum adsorption capacities of MWCNT ( $\mathrm{q}_{\max }$ caffeine $=10.1$ and $\mathrm{q}_{\max }$ diclofenac $=8.5 \mathrm{mg} \mathrm{g}^{-1}$ ) were greatly affected by optimization of adsorption parameters $\left(\mathrm{C}_{0}=15 \mathrm{mg} \mathrm{dm}^{-3}, \mathrm{~m}=50 \mathrm{mg}, \mathrm{t}=30 \mathrm{~min}\right.$, and $\left.\mathrm{T}=25^{\circ} \mathrm{C}\right)$.

The optimization effects of adsorption parameters on the removal of carbamazepine and dorzolamide from water using MWCNT were also investigated by Ncibi et al. (Ncibi and Sillanpää, 2017). The results showed that ultrasound application in the adsorption process improved the $\mathrm{q}_{\max }$ of MWCNT for carbamazepine (224.6 $\left.\mathrm{mg} \mathrm{g}^{-1}\right)$ and dorzolamide $\left(78.8 \mathrm{mg} \mathrm{g}^{-1}\right.$ ) removal (21 and $30 \%$ enhancement) under the optimal conditions.

The MWCNT was prepared with a particle size of $10-40 \mathrm{~nm}$ and a specific surface area of $9.1 \mathrm{~m}^{2} \mathrm{~g}^{-1}$ by ALOthman et al. (ALOthman et al., 2019). The 4-tert-octylphenol endocrine disruptor was adsorbed onto the synthesized MWCNT from the water at a high adsorption rate (94\%); isotherm, kinetic and thermodynamic studies were performed for evaluating the adsorption mechanism. The adsorption results revealed that fast, spontaneous, and exothermic removal of 4-tert-octylphenol occurred onto MWCNT under a liquid film diffusion mechanism.

The adsorption behavior of propranolol (PRO) onto MWCNT was investigated by evaluation of ionic strength, $\mathrm{pH}$, and humic acid (HA) effects on the adsorption process (Nie et al., 2020). Also, the influences of metoprolol tartrate and carbamazepine drugs on PRO removal were evaluated and compared, including the PRO adsorption mechanisms onto MWCNT. The adsorption results exhibited that the PRO adsorption was significantly changed under different pHs, and it was accelerated in the presence of HA without showing any significant changes with varying solution ionic strength. The obtained results of carbamazepine and metoprolol tartrate effects on PRO adsorption showed that the mechanism of PRO adsorption onto MWCNT was mainly attributed to hydrogen bond, and hydrophobic and $\pi-\pi$ EDA interactions.

The mechanism of sulfamethoxazole (SMZ) interaction with various MWCNTs (MWCNT10, MWCNT15, MWCNT15-OH, MWCNT15-COOH, and N-doped MWCNTs) were investigated in the presence of HA in water (Wang et al., 2017). The SMZ adsorption onto CNTs was reduced with the increase of HA concentration due to the binding interaction between free HA and SMZ molecules in water, besides the competitive occupation of MWCNTs adsorption sites by the HA.

The MWCNT was used for the adsorption of PPCPs (acetaminophen, carbendazim, caffeine, and triclosan) from wastewater via pre-coagulation technique (Wang et al., 2018). The wastewater precoagulation effect was evaluated on adsorptive filtration of PPCP by using the MWCNT membrane. Effluent organic matters (biopolymers, and primarily humic substances) in the wastewater competed with PPCPs for adsorption sites of MWCNT. Thus, the MWCNT adsorption capacity was markedly reduced for PPCPs in wastewater remediation. The pre-coagulation of wastewater effectively diminished the competitive adsorption of organic matters, and improved the adsorption efficiency of acetaminophen, carbendazim, caffeine, and triclosan onto MWCNT. These results exhibited that the pre-coagulation initially removed the biopolymers and especially humic substances, then the PPCPs adsorption occurred onto MWCNT. The adsorption capacity of MWCNT-membrane for PPCPs removal was improved with the removal of humic substances; whereas, it was diminished with biopolymer removal due to the reduced membrane permeability and relieved membrane fouling. This finding demonstrated that pre-coagulation is a viable pretreatment method for the filtration of PPCPs by MWCNT from wastewater.

The influence of molecular structure on the sulfonamides sorption affinity (sulfametoxydiazine, sulfamethazine, sulfamerazine, sulfanilamide, sulfadimethoxine, and sulfadiazine) onto hydroxylated MWCNT (HO-MWCNT) was evaluated via batch technique and DFT calculations (Liu et al., 2020). The sulfonamides adsorption onto HO-MWCNT was controlled by chemisorption, and the $\mathrm{q}_{\max }$ of HO-MWCNT for six sulfonamides was in the order: sulfametoxydiazine $>$ sulfamethazine $>$ sulfadiazine $>$ sulfadime thoxine $>$ sulfamerazine $>$ sulfanilamide. The sulfonamides with higher polarity were adsorbed onto polar HO-MWCNT with higher adsorption affinity, based on the similarity and inter-miscibility theory. The results showed that the sulfonamides adsorption onto HO-MWCNT was critically affected by the molecular structure.

The oxidized MWCNT (O-MWCNT) has been synthesized and used for the removal of pefloxacin (PEF) and $\mathrm{Cu}$ (II) from contaminated water (Zhou et al., 2019). The mutual effects of PEF and $\mathrm{Cu}(\mathrm{II})$ adsorption onto O-MWCNT were investigated with single and binary systems by evaluation of adsorption isotherm, kinetic, and 
Table 3

The recent drugs and pesticide adsorption onto carbon nanotubes and graphene-based adsorbents.

\begin{tabular}{|c|c|c|c|c|c|c|c|}
\hline Adsorbent & Adsorbate & Adsorption conditions & $\begin{array}{l}\text { Adsorption } \\
\text { isotherm }\end{array}$ & $\begin{array}{l}\text { Adsorption } \\
\text { kinetic }\end{array}$ & $\begin{array}{l}\mathrm{q}_{\max }(\mathrm{mg} \\
\left.\mathrm{g}^{-1}\right)\end{array}$ & Adsorption mechanism & Reference \\
\hline $\mathrm{GO}^{\mathrm{a}}$ & Ciprofloxacin & $\begin{array}{l}\mathrm{pH}=7.0 \\
\mathrm{C}_{0}=5 \mathrm{mg} \mathrm{L}^{-1} \\
\mathrm{~V}=100 \mathrm{~mL}, \mathrm{~m}=50 \mathrm{mg} \\
\mathrm{T}=298 \mathrm{~K}, \mathrm{t}=50 \mathrm{~min}\end{array}$ & Langmuir & $\begin{array}{l}\text { Pseudo- } \\
\text { second-order }\end{array}$ & 18.65 & Hydrogen bond & Yadav et al. (2018) \\
\hline GO & Norfloxacin & $\begin{array}{l}\mathrm{pH}=7.0 \\
\mathrm{C}_{0}=5 \mathrm{mg} \mathrm{L}^{-1} \\
\mathrm{~V}=100 \mathrm{~mL}, \mathrm{~m}=50 \mathrm{mg} \\
\mathrm{T}=298 \mathrm{~K}, \mathrm{t}=50 \mathrm{~min}\end{array}$ & Langmuir & $\begin{array}{l}\text { Pseudo- } \\
\text { second-order }\end{array}$ & 24.93 & Hydrogen bond & Yadav et al. (2018) \\
\hline GO & Ofloxacin & $\begin{array}{l}\mathrm{pH}=4.0 \\
\mathrm{C}_{0}=5 \mathrm{mg} \mathrm{L}^{-1} \\
\mathrm{~V}=100 \mathrm{~mL}, \mathrm{~m}=50 \mathrm{mg} \\
\mathrm{T}=298 \mathrm{~K}, \mathrm{t}=50 \mathrm{~min}\end{array}$ & Langmuir & $\begin{array}{l}\text { Pseudo- } \\
\text { second-order }\end{array}$ & 40.65 & Hydrogen bond & Yadav et al. (2018) \\
\hline GO & Ciprofloxacin & $\begin{array}{l}\mathrm{pH}=6.0 \\
\mathrm{C}_{0}=-\mathrm{mg} \mathrm{L}^{-1} \\
\mathrm{~V}=10 \mathrm{~mL}, \mathrm{~m}=10 \mathrm{mg} \\
\mathrm{T}=299 \mathrm{~K}, \mathrm{t}=100 \mathrm{~min}\end{array}$ & $\begin{array}{l}\text { Hill } \\
\text { Toth }\end{array}$ & Elovich & 173.4 & $\begin{array}{l}\text { Cation- } \pi \text { bonding } \\
\pi-\pi \text { interaction }\end{array}$ & $\begin{array}{l}\text { Rostamian and } \\
\text { Behnejad (2018) }\end{array}$ \\
\hline GO & Doxycycline & $\begin{array}{l}\mathrm{pH}=6.0 \\
\mathrm{C}_{0}=-\mathrm{mg} \mathrm{L}^{-1} \\
\mathrm{~V}=10 \mathrm{~mL}, \mathrm{~m}=10 \mathrm{mg} \\
\mathrm{T}=299 \mathrm{~K}, \mathrm{t}=200 \mathrm{~min}\end{array}$ & $\begin{array}{l}\text { Hill } \\
\text { Toth }\end{array}$ & $\begin{array}{l}\text { Pseudo- } \\
\text { second-order }\end{array}$ & 131.9 & $\begin{array}{l}\text { Cation- } \pi \text { bonding } \\
\pi-\pi \text { interaction }\end{array}$ & $\begin{array}{l}\text { Rostamian and } \\
\text { Behnejad (2018) }\end{array}$ \\
\hline GO & Tetracycline & $\begin{array}{l}\mathrm{pH}=6.0 \\
\mathrm{C}_{0}=-\mathrm{mg} \mathrm{L}^{-1} \\
\mathrm{~V}=10 \mathrm{~mL}, \mathrm{~m}=10 \mathrm{mg} \\
\mathrm{T}=299 \mathrm{~K}, \mathrm{t}=200 \mathrm{~min}\end{array}$ & $\begin{array}{l}\text { Hill } \\
\text { Toth }\end{array}$ & $\begin{array}{l}\text { Pseudo- } \\
\text { second-order }\end{array}$ & 96.98 & $\begin{array}{l}\text { Cation- } \pi \text { bonding } \\
\pi-\pi \text { interaction }\end{array}$ & $\begin{array}{l}\text { Rostamian and } \\
\text { Behnejad (2018) }\end{array}$ \\
\hline GO & Sodium diclofenac & $\begin{array}{l}\mathrm{pH}=6.2 \\
\mathrm{C}_{0}=10 \mathrm{mg} \mathrm{L}^{-1} \\
\mathrm{~V}=-\mathrm{mL}, \mathrm{m}=250 \mathrm{mg} \mathrm{L}^{-1} \\
\mathrm{~T}=298 \mathrm{~K}, \mathrm{t}=300 \mathrm{~min}\end{array}$ & Langmuir & $\begin{array}{l}\text { Pseudo- } \\
\text { second-order }\end{array}$ & 128.74 & $\begin{array}{l}\text { Electrostatic attraction } \\
\text { Hydrogen bond } \\
\text { Hydrophobic interaction } \\
\pi-\pi \text { bond }\end{array}$ & Guerra et al. (2019) \\
\hline GO & Metformin & $\begin{array}{l}\mathrm{pH}=6.26 \\
\mathrm{C}_{0}=521 \mathrm{mg} \mathrm{L}^{-1} \\
\mathrm{~V}=-\mathrm{mL}, \mathrm{m}=126.02 \mathrm{mg} \mathrm{L}^{-1} \\
\mathrm{~T}=318.5 \mathrm{~K}, \mathrm{t}=-\mathrm{min}\end{array}$ & $\begin{array}{l}\text { Sips } \\
\text { Hill }\end{array}$ & $\begin{array}{l}\text { Pseudo-first- } \\
\text { order }\end{array}$ & 122.61 & $\begin{array}{l}\text { Van der Waals forces } \\
\pi-\pi \text { interactions }\end{array}$ & $\begin{array}{l}\text { Balasubramani et al. } \\
(2020)\end{array}$ \\
\hline GO & Cephalexin & $\begin{array}{l}\mathrm{pH}=7.0 \\
\mathrm{C}_{0}=100 \mathrm{mg} \mathrm{L}^{-1} \\
\mathrm{~V}=25 \mathrm{~mL}, \mathrm{~m}=10 \mathrm{mg} \\
\mathrm{T}=298 \mathrm{~K}, \mathrm{t}=420 \mathrm{~min}\end{array}$ & Langmuir & $\begin{array}{l}\text { Pseudo- } \\
\text { second-order }\end{array}$ & 164 & - & Wernke et al. (2020) \\
\hline GO & Trimethoprim & $\begin{array}{l}\mathrm{pH}=8.0 \\
\mathrm{C}_{0}=50 \mathrm{mg} \mathrm{L}-1 \\
\mathrm{~V}=-\mathrm{mL}, \mathrm{m}=15 \mathrm{mg} \\
\mathrm{T}=298 \mathrm{~K}, \mathrm{t}=60 \mathrm{~min}\end{array}$ & Freundlich & $\begin{array}{l}\text { Pseudo- } \\
\text { second-order }\end{array}$ & 204.08 & $\begin{array}{l}\text { Cation- } \pi \text { and } \pi-\pi \text { dispersion } \\
\text { interactions }\end{array}$ & $\begin{array}{l}\text { Çalışkan Salihi et al. } \\
(2020)\end{array}$ \\
\hline GO & Isoniazid & $\begin{array}{l}\mathrm{pH}=2.0 \\
\mathrm{C}_{0}=25 \mathrm{mg} \mathrm{L} \\
\mathrm{V}=-\mathrm{mL}, \mathrm{m}=15 \mathrm{mg} \\
\mathrm{T}=298 \mathrm{~K}, \mathrm{t}=60 \mathrm{~min}\end{array}$ & Langmuir & $\begin{array}{l}\text { Intraparticle } \\
\text { diffusion }\end{array}$ & 13.89 & Electrostatic attraction & $\begin{array}{l}\text { Çalışkan Salihi et al. } \\
(2020)\end{array}$ \\
\hline GO & Norfloxacin & $\begin{array}{l}\mathrm{pH}=7.0 \\
\mathrm{C}_{0}=30 \mathrm{mg} \mathrm{L}^{-1} \\
\mathrm{~V}=10 \mathrm{~mL}, \mathrm{~m}=200 \mathrm{mg} \mathrm{L}^{-1} \\
\mathrm{~T}=303 \mathrm{~K}, \mathrm{t}=30 \mathrm{~min}\end{array}$ & Sips & $\begin{array}{l}\text { Pseudo- } \\
\text { second-order }\end{array}$ & 374.9 & $\begin{array}{l}\text { Van der Waals forces } \\
\pi-\pi \text { interaction } \\
\text { Hydrogen bond }\end{array}$ & Moreira et al. (2020) \\
\hline GO & Naproxen & $\begin{array}{l}\mathrm{pH}=4.0 \\
\mathrm{C}_{0}=10 \mathrm{mg} \mathrm{L}-1 \\
\mathrm{~V}=-\mathrm{mL}, \mathrm{m}=30 \mathrm{mg} \\
\mathrm{T}=298 \mathrm{~K}, \mathrm{t}=60 \mathrm{~min}\end{array}$ & $\begin{array}{l}\text { Dubinin- } \\
\text { Radushkevich }\end{array}$ & $\begin{array}{l}\text { Pseudo- } \\
\text { second-order }\end{array}$ & 15.16 & Chemical adsorption & Ciğeroğlu et al. (2020) \\
\hline $\mathrm{PG}^{\mathrm{b}}$ & Atenolol & $\begin{array}{l}\mathrm{pH}=7.5 \\
\mathrm{C}_{0}=10 \mathrm{mg} \mathrm{L} \\
\mathrm{V}=20 \mathrm{~mL}, \mathrm{~m}=5 \mathrm{mg} \\
\mathrm{T}=295 \mathrm{~K}, \mathrm{t}=120 \mathrm{~min}\end{array}$ & Toth & $\begin{array}{l}\text { Pseudo-first- } \\
\text { order }\end{array}$ & 1455.4 & Physical adsorption & Khalil et al. (2020) \\
\hline PG & Carbamazepine & $\begin{array}{l}\mathrm{pH}=7.5 \\
\mathrm{C}_{0}=10 \mathrm{mg} \mathrm{L} \\
\mathrm{V}=20 \mathrm{~mL}, \mathrm{~m}=5 \mathrm{mg} \\
\mathrm{T}=295 \mathrm{~K}, \mathrm{t}=120 \mathrm{~min}\end{array}$ & Freundlich & $\begin{array}{l}\text { Pseudo- } \\
\text { second-order }\end{array}$ & 191.67 & Physical adsorption & Khalil et al. (2020) \\
\hline PG & Ciprofloxacin & $\begin{array}{l}\mathrm{pH}=7.5 \\
\mathrm{C}_{0}=10 \mathrm{mg} \mathrm{L} \\
\mathrm{V}=20 \mathrm{~mL}, \mathrm{~m}=5 \mathrm{mg} \\
\mathrm{T}=295 \mathrm{~K}, \mathrm{t}=120 \mathrm{~min}\end{array}$ & Temkin & $\begin{array}{l}\text { Pseudo- } \\
\text { second-order }\end{array}$ & 409.15 & Physical adsorption & Khalil et al. (2020) \\
\hline PG & Diclofenac & $\begin{array}{l}\mathrm{pH}=7.5 \\
\mathrm{C}_{0}=10 \mathrm{mg} \mathrm{L} \\
\mathrm{V}=20 \mathrm{~mL}, \mathrm{~m}=5 \mathrm{mg} \\
\mathrm{T}=295 \mathrm{~K}, \mathrm{t}=120 \mathrm{~min}\end{array}$ & Sips & $\begin{array}{l}\text { Pseudo- } \\
\text { second-order }\end{array}$ & 91.59 & Physical adsorption & Khalil et al. (2020) \\
\hline PG & Gemfibrozil & $\begin{array}{l}\mathrm{pH}=7.5 \\
\mathrm{C}_{0}=10 \mathrm{mg} \mathrm{L}^{-1} \\
\mathrm{~V}=20 \mathrm{~mL}, \mathrm{~m}=5 \mathrm{mg} \\
\mathrm{T}=295 \mathrm{~K}, \mathrm{t}=120 \mathrm{~min}\end{array}$ & Sips & $\begin{array}{l}\text { Pseudo- } \\
\text { second-order }\end{array}$ & 160.86 & Physical adsorption & Khalil et al. (2020) \\
\hline
\end{tabular}


Table 3 (continued)

\begin{tabular}{|c|c|c|c|c|c|c|c|}
\hline Adsorbent & Adsorbate & Adsorption conditions & $\begin{array}{l}\text { Adsorption } \\
\text { isotherm }\end{array}$ & $\begin{array}{l}\text { Adsorption } \\
\text { kinetic }\end{array}$ & $\begin{array}{l}\mathrm{q}_{\max }(\mathrm{mg} \\
\left.\mathrm{g}^{-1}\right)\end{array}$ & Adsorption mechanism & Reference \\
\hline PG & Ibuprofen & $\begin{array}{l}\mathrm{pH}=7.5 \\
\mathrm{C}_{0}=10 \mathrm{mg} \mathrm{L} \\
\mathrm{V}=20 \mathrm{~mL}, \mathrm{~m}=5 \mathrm{mg} \\
\mathrm{T}=295 \mathrm{~K}, \mathrm{t}=120 \mathrm{~min}\end{array}$ & Langmuir & $\begin{array}{l}\text { Pseudo- } \\
\text { second-order }\end{array}$ & 110.30 & Physical adsorption & Khalil et al. (2020) \\
\hline GO-IL & Sulfamethoxazole & $\begin{array}{l}\mathrm{pH}=6.28 \\
\mathrm{C}_{0}=10 \mathrm{mg} \mathrm{L}^{-1} \\
\mathrm{~V}=10 \mathrm{~mL}, \mathrm{~m}=0.9 \mathrm{~g} \mathrm{~L}^{-1} \\
\mathrm{~T}=299 \mathrm{~K}, \mathrm{t}=60 \mathrm{~min}^{-1}\end{array}$ & Freundlich & $\begin{array}{l}\text { Pseudo- } \\
\text { second-order }\end{array}$ & 27.25 & $\begin{array}{l}\text { Hydrophobic interaction } \\
\pi-\pi \text { interaction } \\
\text { Hydrogen bond } \\
\text { Electrostatic attraction }\end{array}$ & Ogunleye et al. (2020) \\
\hline GO & Carbamazepine & $\begin{array}{l}\mathrm{pH}=2.0 \\
\mathrm{C}_{0}=50 \mathrm{mg} \mathrm{L}^{-1} \\
\mathrm{~V}=-\mathrm{mL}, \mathrm{m}=10 \mathrm{mg} \\
\mathrm{T}=299 \mathrm{~K}, \mathrm{t}=30 \mathrm{~min}\end{array}$ & Langmuir & $\begin{array}{l}\text { Pseudo- } \\
\text { second-order }\end{array}$ & 28.54 & $\begin{array}{l}\pi-\pi \text { interactions } \\
\text { Hydrophobic interaction }\end{array}$ & Lawal et al. (2020) \\
\hline GO-IL & Carbamazepine & $\begin{array}{l}\mathrm{pH}=2.0 \\
\mathrm{C}_{0}=50 \mathrm{mg} \mathrm{L}^{-1} \\
\mathrm{~V}=-\mathrm{mL}, \mathrm{m}=10 \mathrm{mg} \\
\mathrm{T}=299 \mathrm{~K}, \mathrm{t}=10 \mathrm{~min}\end{array}$ & $\begin{array}{l}\text { Redlich- } \\
\text { Peterson }\end{array}$ & $\begin{array}{l}\text { Pseudo- } \\
\text { second-order }\end{array}$ & 61.88 & $\begin{array}{l}\pi-\pi \text { interactions } \\
\text { Hydrophobic interaction }\end{array}$ & Lawal et al. (2020) \\
\hline GO & Ketoprofen & $\begin{array}{l}\mathrm{pH}=4.0 \\
\mathrm{C}_{0}=50 \mathrm{mg} \mathrm{L}^{-1} \\
\mathrm{~V}=-\mathrm{mL}, \mathrm{m}=10 \mathrm{mg}\end{array}$ & Langmuir & $\begin{array}{l}\text { Pseudo- } \\
\text { second-order }\end{array}$ & 16.45 & $\begin{array}{l}\pi-\pi \text { interactions } \\
\text { Hydrophobic } \\
\text { Interaction }\end{array}$ & Lawal et al. (2020) \\
\hline GO-IL & Ketoprofen & $\begin{array}{l}\mathrm{T}=299 \mathrm{~K}, \mathrm{t}=30 \mathrm{~min} \\
\mathrm{pH}=8.0 \\
\mathrm{C}_{0}=50 \mathrm{mg} \mathrm{L}^{-1} \\
\mathrm{~V}=-\mathrm{mL}, \mathrm{m}=10 \mathrm{mg} \\
\mathrm{T}=299 \mathrm{~K}, \mathrm{t}=10 \mathrm{~min}\end{array}$ & $\begin{array}{l}\text { Redlich- } \\
\text { Peterson }\end{array}$ & $\begin{array}{l}\text { Pseudo- } \\
\text { second-order }\end{array}$ & 87.88 & $\begin{array}{l}\text { Electrostatic attraction } \\
\pi-\pi \text { interactions } \\
\text { Hydrophobic interaction } \\
\text { Electrostatic attraction }\end{array}$ & Lawal et al. (2020) \\
\hline GO & Sulfamethoxazole & $\begin{array}{l}\mathrm{pH}=4.0 \\
\mathrm{C}_{0}=50 \mathrm{mg} \mathrm{L} \\
\mathrm{V}=-\mathrm{mL}, \mathrm{m}=10 \mathrm{mg} \\
\mathrm{T}=299 \mathrm{~K}, \mathrm{t}=30 \mathrm{~min}\end{array}$ & Langmuir & $\begin{array}{l}\text { Pseudo- } \\
\text { second-order }\end{array}$ & 21.33 & $\begin{array}{l}\pi-\pi \text { interactions } \\
\text { Hydrophobic } \\
\text { Interaction } \\
\text { Electrostatic attraction }\end{array}$ & Lawal et al. (2020) \\
\hline GO-IL & Sulfamethoxazole & $\begin{array}{l}\mathrm{pH}=6.0 \\
\mathrm{C}_{0}=50 \mathrm{mg} \mathrm{L}^{-1} \\
\mathrm{~V}=-\mathrm{mL}, \mathrm{m}=10 \mathrm{mg}\end{array}$ & $\begin{array}{l}\text { Redlich- } \\
\text { Peterson }\end{array}$ & Weber-Morris & 99.16 & $\begin{array}{l}\pi-\pi \text { interactions } \\
\text { Hydrophobic } \\
\text { Interaction }\end{array}$ & Lawal et al. (2020) \\
\hline MWCNT $^{\mathrm{d}}$ & Propranolol & $\begin{array}{l}\mathrm{T}=299 \mathrm{~K}, \mathrm{t}=10 \mathrm{~min} \\
\mathrm{pH}=6.5 \\
\mathrm{C}_{0}=25 \mathrm{mg} \mathrm{L}^{-1} \\
\mathrm{~V}=40 \mathrm{~mL}, \mathrm{~m}=10 \mathrm{mg} \\
\mathrm{T}=299 \mathrm{~K}, \mathrm{t}=30 \mathrm{~min}\end{array}$ & Sips & $\begin{array}{l}\text { Pseudo- } \\
\text { second-order }\end{array}$ & 380.22 & $\begin{array}{l}\text { Electrostatic attraction } \\
\pi-\pi \text { EDA interaction } \\
\text { Hydrophobic interaction } \\
\text { Hydrogen bond }\end{array}$ & Nie et al. (2020) \\
\hline $\mathrm{N}-\mathrm{CNT}^{\mathrm{e}}$ & Dimetridazole & $\begin{array}{l}\mathrm{pH}=7.0 \\
\mathrm{C}_{0}=-\mathrm{mg} \mathrm{L}^{-1} \\
\mathrm{~V}=40 \mathrm{~mL}, \mathrm{~m}=10 \mathrm{mg} \\
\mathrm{T}=299 \mathrm{~K}, \mathrm{t}=40 \mathrm{~min}\end{array}$ & - & - & 40.93 & $\begin{array}{l}\pi-\pi \text { dispersive interactions } \\
\text { Electrostatic interaction } \\
\text { hydrophobic } \\
\text { interactions }\end{array}$ & $\begin{array}{l}\text { Carrales-Alvarado et al. } \\
(2020)\end{array}$ \\
\hline SWCNT $^{\mathrm{f}}$ & Dimetridazole & $\begin{array}{l}\mathrm{pH}=7.0 \\
\mathrm{C}_{0}=-\mathrm{mg} \mathrm{L}^{-1} \\
\mathrm{~V}=40 \mathrm{~mL}, \mathrm{~m}=10 \mathrm{mg} \\
\mathrm{T}=299 \mathrm{~K}, \mathrm{t}=40 \mathrm{~min}\end{array}$ & - & - & 81.86 & $\begin{array}{l}\pi-\pi \text { dispersive interactions } \\
\text { Electrostatic interaction } \\
\text { hydrophobic } \\
\text { interactions }\end{array}$ & $\begin{array}{l}\text { Carrales-Alvarado et al. } \\
(2020)\end{array}$ \\
\hline MWCNT & Dimetridazole & $\begin{array}{l}\mathrm{pH}=7.0 \\
\mathrm{C}_{0}=-\mathrm{mg} \mathrm{L}^{-1} \\
\mathrm{~V}=40 \mathrm{~mL}, \mathrm{~m}=10 \mathrm{mg} \\
\mathrm{T}=299 \mathrm{~K}, \mathrm{t}=40 \mathrm{~min}\end{array}$ & $\longrightarrow$ & - & 45.16 & $\begin{array}{l}\pi-\pi \text { dispersive interactions } \\
\text { Electrostatic interaction } \\
\text { hydrophobic } \\
\text { interactions }\end{array}$ & $\begin{array}{l}\text { Carrales-Alvarado et al. } \\
(2020)\end{array}$ \\
\hline $\begin{array}{l}\text { MWCNT- } \\
\mathrm{COOH}^{\mathrm{g}}\end{array}$ & Dimetridazole & $\begin{array}{l}\mathrm{pH}=7.0 \\
\mathrm{C}_{0}=-\mathrm{mg} \mathrm{L}^{-1} \\
\mathrm{~V}=40 \mathrm{~mL}, \mathrm{~m}=10 \mathrm{mg} \\
\mathrm{T}=299 \mathrm{~K}, \mathrm{t}=40 \mathrm{~min}\end{array}$ & - & - & 32.46 & $\begin{array}{l}\pi-\pi \text { dispersive interactions } \\
\text { Electrostatic interaction } \\
\text { hydrophobic } \\
\text { interactions }\end{array}$ & $\begin{array}{l}\text { Carrales-Alvarado et al. } \\
(2020)\end{array}$ \\
\hline $\mathrm{N}-\mathrm{CNT}$ & Metronidazole & $\begin{array}{l}\mathrm{pH}=7.0 \\
\mathrm{C}_{0}=-\mathrm{mg} \mathrm{L}^{-1} \\
\mathrm{~V}=40 \mathrm{~mL}, \mathrm{~m}=10 \mathrm{mg} \\
\mathrm{T}=299 \mathrm{~K}, \mathrm{t}=40 \mathrm{~min}\end{array}$ & - & - & 56.49 & $\begin{array}{l}\pi-\pi \text { dispersive interactions } \\
\text { Electrostatic interaction } \\
\text { hydrophobic } \\
\text { interactions }\end{array}$ & $\begin{array}{l}\text { Carrales-Alvarado et al. } \\
(2020)\end{array}$ \\
\hline SWCNT & Metronidazole & $\begin{array}{l}\mathrm{pH}=7.0 \\
\mathrm{C}_{0}=-\mathrm{mg} \mathrm{L}^{-1} \\
\mathrm{~V}=40 \mathrm{~mL}, \mathrm{~m}=10 \mathrm{mg} \\
\mathrm{T}=299 \mathrm{~K}, \mathrm{t}=40 \mathrm{~min}\end{array}$ & $\longrightarrow$ & - & 100.98 & $\begin{array}{l}\pi-\pi \text { dispersive interactions } \\
\text { Electrostatic interaction } \\
\text { hydrophobic } \\
\text { interactions }\end{array}$ & $\begin{array}{l}\text { Carrales-Alvarado et al. } \\
(2020)\end{array}$ \\
\hline MWCNT & Metronidazole & $\begin{array}{l}\mathrm{pH}=7.0 \\
\mathrm{C}_{0}=-\mathrm{mg} \mathrm{L}^{-1} \\
\mathrm{~V}=40 \mathrm{~mL}, \mathrm{~m}=10 \mathrm{mg} \\
\mathrm{T}=299 \mathrm{~K}, \mathrm{t}=40 \mathrm{~min}\end{array}$ & - & - & 46.21 & $\begin{array}{l}\pi-\pi \text { dispersive interactions } \\
\text { Electrostatic interaction } \\
\text { hydrophobic } \\
\text { interactions }\end{array}$ & $\begin{array}{l}\text { Carrales-Alvarado et al. } \\
(2020)\end{array}$ \\
\hline $\begin{array}{l}\text { MWCNT- } \\
\text { COOH }\end{array}$ & Metronidazole & $\begin{array}{l}\mathrm{pH}=7.0 \\
\mathrm{C}_{0}=-\mathrm{mg} \mathrm{L}^{-1} \\
\mathrm{~V}=40 \mathrm{~mL}, \mathrm{~m}=10 \mathrm{mg} \\
\mathrm{T}=299 \mathrm{~K}, \mathrm{t}=40 \mathrm{~min}\end{array}$ & - & - & 46.21 & $\begin{array}{l}\pi-\pi \text { dispersive interactions } \\
\text { Electrostatic interaction } \\
\text { hydrophobic } \\
\text { interactions }\end{array}$ & $\begin{array}{l}\text { Carrales-Alvarado et al. } \\
(2020)\end{array}$ \\
\hline $\begin{array}{l}\text { MWCNT- } \\
\mathrm{OH}^{\mathrm{h}}\end{array}$ & Sulfanilamide & $\begin{array}{l}\mathrm{pH}=3.0 \\
\mathrm{C}_{0}=20 \mathrm{mg} \mathrm{L}^{-1} \\
\mathrm{~V}=50 \mathrm{~mL}, \mathrm{~m}=1 \mathrm{~g} \mathrm{~L}^{-1} \\
\mathrm{~T}=298 \mathrm{~K}, \mathrm{t}=20 \mathrm{~min}\end{array}$ & Langmuir & General order & 67.15 & $\begin{array}{l}\text { Electrostatic attraction } \\
\text { Hydrogen bond } \\
\text { EDA interaction } \\
\text { Lewis acid-base interaction }\end{array}$ & Liu et al. (2020) \\
\hline $\begin{array}{l}\text { MWCNT- } \\
\mathrm{OH}\end{array}$ & Sulfamerazine & $\begin{array}{l}\mathrm{pH}=3.0 \\
\mathrm{C}_{0}=20 \mathrm{mg} \mathrm{L}^{-1}\end{array}$ & Liu & $\begin{array}{l}\text { Pseudo- } \\
\text { second-order }\end{array}$ & 115.74 & $\begin{array}{l}\text { Electrostatic attraction } \\
\text { Hydrogen bond }\end{array}$ & Liu et al. (2020) \\
\hline
\end{tabular}


Table 3 (continued)

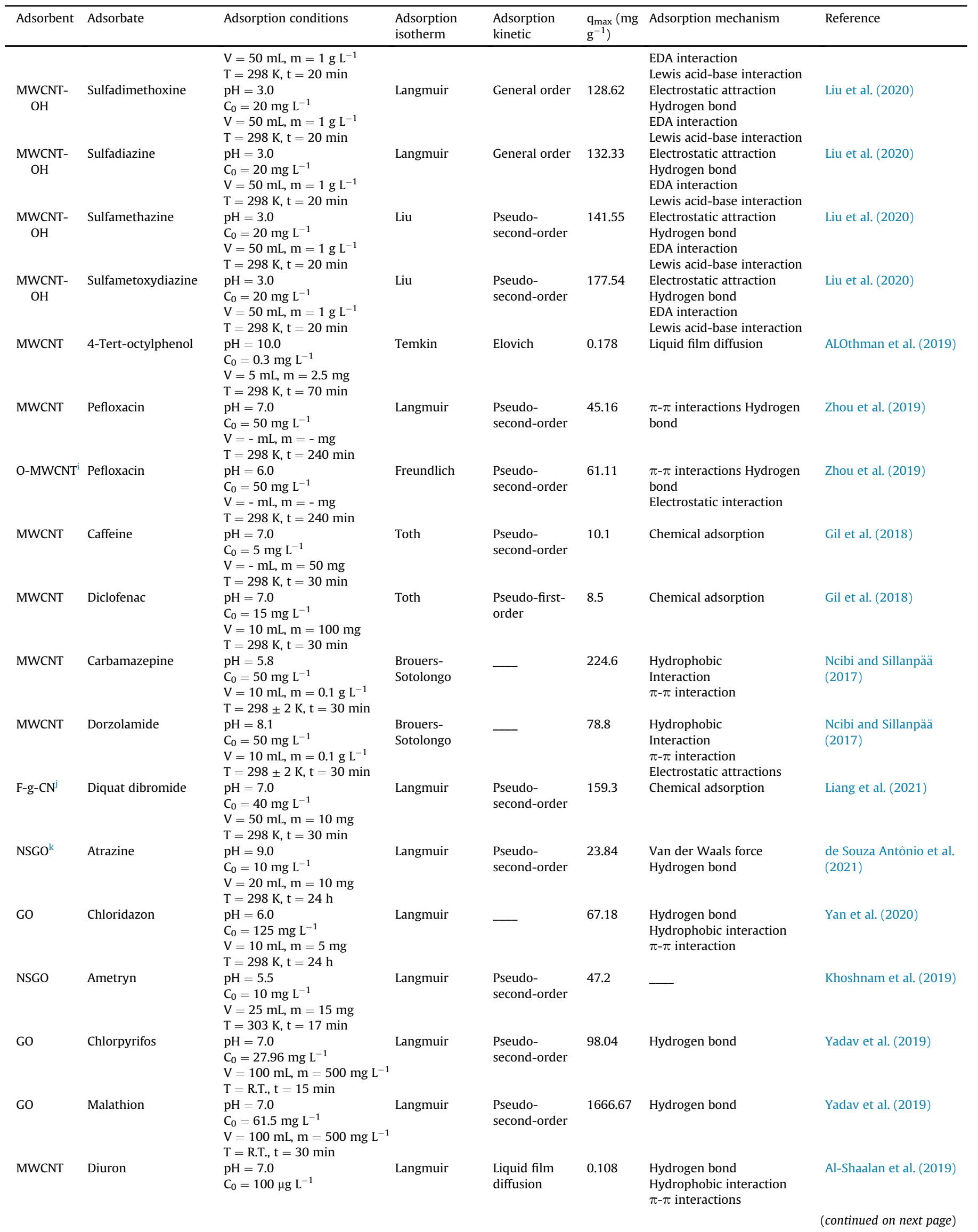


Table 3 (continued)

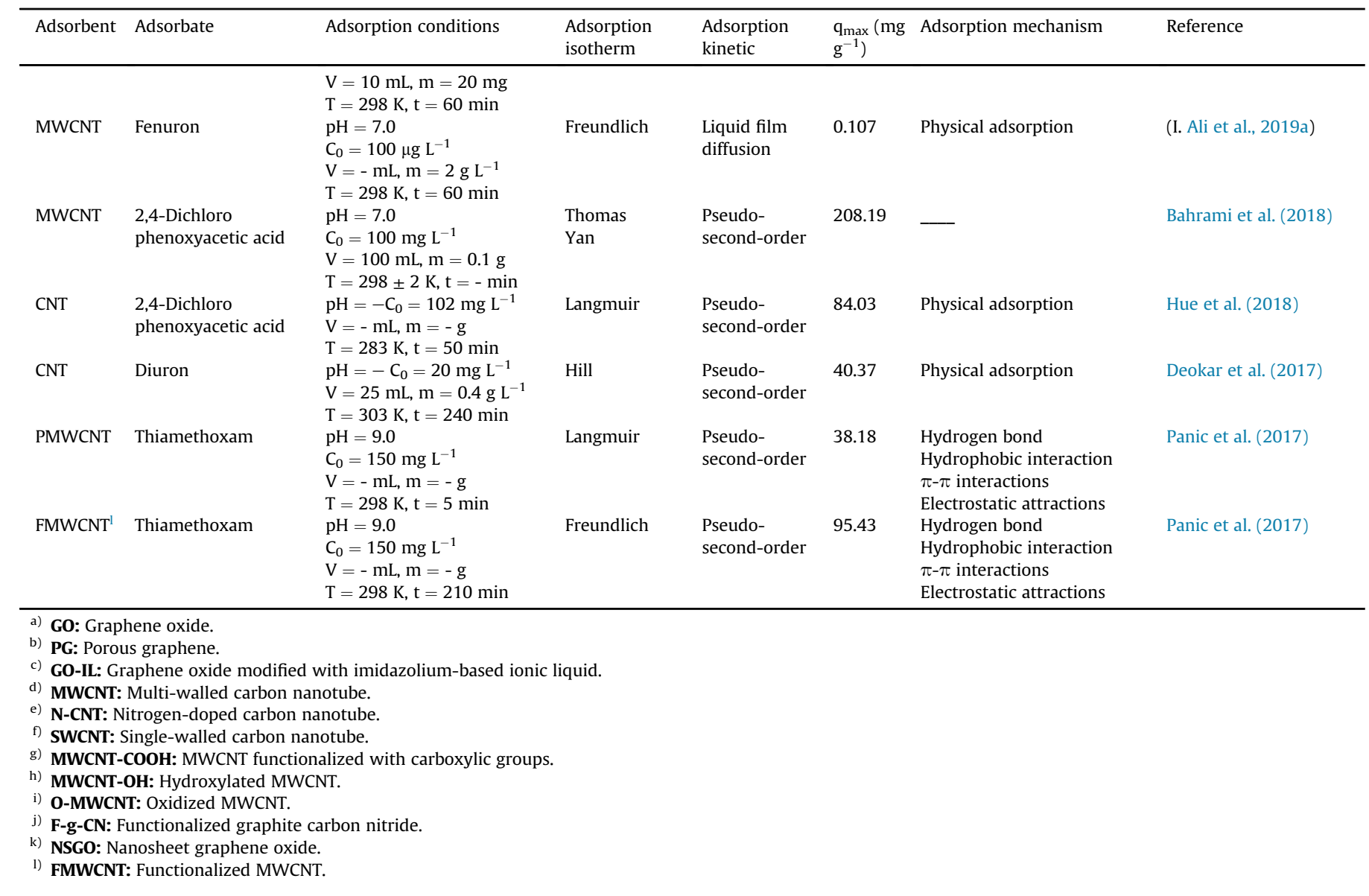

thermodynamic. There were site enhancement and competition between PEF and $\mathrm{Cu}(\mathrm{II})$ on O-MWCNT in a binary system; removal of $\mathrm{PEF} / \mathrm{Cu}$ (II) was promoted at a low concentration of $\mathrm{PEF} / \mathrm{Cu}$ (II) $(\leq 25 \mathrm{ppm})$ and was inhibited at a higher concentration. The results exhibited that the $\mathrm{PEF} / \mathrm{Cu}(\mathrm{II})$ functioned as a bridge between $\mathrm{O}$ MWCNT and PEF/Cu(II). The PEF was adsorbed onto O-MWCNT with hydrogen bond and $\pi-\pi$ interactions.

The influence of surface morphology and chemistry of CNTs (SWCNT, CNT doped with nitrogen: $N$-CNT, MWCNT, and carboxylic acid functionalized MWCNT: MWCNT-COOH) on their sorption affinity towards metronidazole and dimetridazole from aqueous solutions has been studied (Carrales-Alvarado et al., 2020). The adsorption efficiency relied remarkably on the surface area $\left(\mathrm{S}_{\mathrm{BET}}\right.$ SWCNT $=404, \mathrm{~S}_{\mathrm{BET}} N-C N T=371, \mathrm{~S}_{\mathrm{BET}} \mathrm{MWCNT}=144$, and $\mathrm{S}_{\mathrm{BET}}$ MWCNT-COOH $=115 \mathrm{~m}^{2} \mathrm{~g}^{-1}$ ) and declined linearly with the concentration of carboxylic and phenolic sites per unit surface area. The SWCNT, with the highest surface area and basic surface in comparison to other CNTs with the acidic surface, presented the highest $\mathrm{q}_{\max }$ towards dimetridazole $\left(84 \mathrm{mg} \mathrm{g}^{-1}\right)$ and metronidazole (101 $\left.\mathrm{mg} \mathrm{g}^{-1}\right)$; adsorption of dimetridazole and metronidazole onto CNTs was mainly attributed to the $\pi-\pi$ stacking interactions, which were promoted by basic groups.

The O-MWCNT has been deployed as an adsorbent for the miniaturized solid-phase extraction of progestins (19norethisterone acetate, medroxyprogesterone acetate, and levonorgestrel) from the water before their HPLC-UV determination (Aguinaga Martínez et al., 2020). The O-MWCNT with polar functional groups exhibited an excellent adsorption performance for progestins. Attractive features of solid-phase extraction method for the adsorption of progestins onto O-MWCNT included low usage of organic solvents and the pre-concentration factor (up to 100).

The pristine and $\mathrm{HCl}$-treated MWCNTs have been used as solidphase extraction adsorbent for the separation and enrichment of pharmaceuticals (azithromycin, bisoprolol, carbamazepine, cilazapril, clopidogrel, diazepam, diclofenac, enalapril, erythromycin, lorazepam, metoprolol, simvastatin, sulfamethoxazole, 4acetylaminoantipyrine, and 4-formylaminoantipyrine) from the surface and ground waters, before liquid chromatography-tandem mass spectrometry analysis (Lalović et al., 2017). The adsorption efficiency of selected pharmaceuticals was favorably affected by the chemical treatment of MWCNT due to a decrease in the electron acceptor groups of treated MWCNT surface.

The molecular dynamics simulations in water were applied for the adsorption of ciprofloxacin in the neutral and zwitterionic forms onto SWCNT (Veclani and Melchior, 2020). The strongly negative values of interaction energies $\left(E_{\text {int }}\right)$ suggested preferential sorption on the SWCNT internal wall. Also, the calculated free energy exhibited that the ciprofloxacin adsorption spontaneously occurred onto SWCNT; adsorbed ciprofloxacins were frequently oriented parallel to the interacting surface through $\pi-\pi$ interactions. The ciprofloxacin-ciprofloxacin $\mathrm{H}$-bonds analysis in water showed that ciprofloxacin was dispersed on the SWCNT. However, eight molecules of ciprofloxacin were gathered inside the SWCNT, which resulted in some aggregation.

4.2.3.2. Adsorption of pesticides onto carbon nanotubes. Besides drugs, pesticides are another class of pollutants that have been extensively removed using CNTs in aqueous solutions. The 
synthesized CNT from plastic waste was applied as an effective adsorbent for the diuron elimination from aqueous media (Deokar et al., 2017). This research established that the plastic waste derived CNT with $\mathrm{q}_{\max }=103.73 \mathrm{mg} \mathrm{g}^{-1}$ could be a suitable adsorbent for the water treatment.

The low-cost MWCNT has been prepared by using $\mathrm{Ni} / \mathrm{MgO}$ compound in particle sizes ranging from 10.0 to $40.0 \mathrm{~nm}$ and a surface area of $9.10 \mathrm{~m}^{2} \mathrm{~g}^{-1}$ (Al-Shaalan et al., 2019). Efficient diuron removal (90.5\%) was achieved using the synthesized MWCT $\left(\mathrm{q}_{\max }=132.5 \mathrm{mg} \mathrm{g}^{-1}\right)$ from water at optimized conditions $\left(C_{\text {diuron }}=100.0 \mu \mathrm{g} \mathrm{L}^{-1}, \mathrm{t}=60 \mathrm{~min}, \mathrm{C}_{\mathrm{MWCT}}=2.0 \mathrm{~g} \mathrm{~L}^{-1}, \mathrm{pH}=7.0\right.$, and $\mathrm{T}=25^{\circ} \mathrm{C}$ ). The statistical adsorption data confirmed the prompt adsorption of diuron onto MWCNT in an endothermic manner. The supra-molecular adsorption mechanism was designated by modeling readings and calculating the energy and binding affinity of diuron with MWCNTs. The interactions between diutron and MWCNT involved the $\pi$-donor hydrogen bonds, $\pi$-alkyl bonds, $\pi-\pi$ stacked, and $\pi-\pi$ T-shaped bonds. These results established that the physisorption of diuron and the creation of diutron-MWCNT composite comprised sixteen hydrophobic connections and two hydrogen bonds.

Likewise, the $\mathrm{Ni} / \mathrm{MgO}$-derived MWCNT has been deployed for the adsorption of fenuron from water through simulation studies for mechanistic insight (I. Ali et al., 2019a). The results revealed that the physisorption of fenuron occurred onto synthesized MCNT, and there was one $\pi$ - $\pi$ T-shaped, seven $\pi-\pi$ stacked, three $\pi$-alkyl, and one $\pi-\sigma$ type of hydrophobic interactions between fenuron and MWCNT.

In another study, the isotherm, kinetic, and thermodynamic of thiamethoxam adsorption onto MWCNT were evaluated by Pani et al. for predicting the adsorption mechanism (Panic et al., 2017). To clarify the effect of nanotube structure on the sorption mechanism, the adsorption process was evaluated for both the pristine and nitric acid-modified MWCNT. The introduction of functional groups possessing an oxygen atom in the structure of MWCNT positively affected the thiamethoxam removal from water. Based on the adsorption results, the thiamethoxam removal by MWCNTs occurred with physicochemical properties, and the electron donoracceptor interactions (aromatic $\pi-\pi$ interactions) being the dominant mechanism for the adsorption of thiamethoxam onto MWCNTs. The thermodynamic data showed the spontaneous and exothermic nature of thiamethoxam removal, regardless of the MWCNTs type.

The optimization of malathion removal from water using MWCNT has been reported by Dehghani et al. (2017). Based on the obtained results from both the statistical and three-factor response surface modeling (RSM) analyses for 26 steps of experiments, the MWCNTs could be efficiently applied for the removal of $100 \%$ malathion at the optimal conditions $\left(C_{0}=6 \mathrm{mg} \mathrm{L}^{-1}\right.$, $\mathrm{C}_{\mathrm{MWCNT}}=0.5 \mathrm{~g} \mathrm{~L}^{-1}, \mathrm{t}=30 \mathrm{~min}, \mathrm{pH}=7$, and $\mathrm{T}=24 \pm 2{ }^{\circ} \mathrm{C}$ ).

Also, the effect of adsorption optimization on the diazinon removal was investigated using MWCNT from the water in a batch technique (Dehghani et al., 2019). Optimizing of the adsorption parameters showed that MWCNT adsorbed $100 \%$ of diazinon from water under optimal conditions $\left(C_{\text {diazinon }}=0.3 \mathrm{mg} \mathrm{L}^{-1}\right.$, $\mathrm{C}_{\text {MWCNT }}=0.1 \mathrm{~g} \mathrm{~L}^{-1}, \mathrm{t}=15 \mathrm{~min}$ at $\mathrm{pH}=4$ and 7). The MWCNT efficiency for diazinon adsorption in acidic $\mathrm{pH}$ could be improved because of the hydroxyl group's protonation.

The adsorption of 2,4-D from aqueous solution onto CNT $\left(S_{\mathrm{BET}}=267 \mathrm{~m}^{2} \mathrm{~g}^{-1}\right)$ was investigated via batch technique (Hue et al., 2018). The spontaneous and exothermic adsorption of 2,4-D has occurred onto CNT with $\mathrm{q}_{\max }=84.03 \mathrm{mg} \mathrm{g}^{-1}$ under optimal conditions $\left(\mathrm{C}_{0}=102 \mathrm{mg} \mathrm{L}^{-1}, \mathrm{t}=50 \mathrm{~min}\right.$, and $\left.\mathrm{T}=283 \mathrm{~K}\right)$. Likewise, the performance of MWCNT $\left(\mathrm{S}_{\mathrm{BET}}=200 \mathrm{~m}^{2} \mathrm{~g}^{-1}\right.$ and $\mathrm{pH}$ PZC $\left.=4.1\right)$ was explored for the adsorption of 2,4-D in a fixed-bed column system
(Bahrami et al., 2018). The higher removal of 2,4-D ( $\mathrm{pKa}=2.64$ ) occurred at the smaller flow rate $\left(0.5 \mathrm{~mL} \mathrm{~min}^{-1}\right)$, lower concentration of 2,4-D (50 mg L $\left.{ }^{-1}\right)$, larger bed depth $(9 \mathrm{~cm})$, and lower $\mathrm{pH}$ (2). The results established that the MWCNTs could be an excellent choice for the adsorption of pesticide but the adsorption equilibrium time is a significant factor.

Documented literature described the adsorption of chlorophenols (2,4,6-trichlorophenol: 2,4,6-TClP, 2,6-dichlorophenol: 2,6-DClP, 3,4-dichlorophenol: 3,4-DClP, 2,4-dichlorophenol: 2,4DCIP, 4-chlorophenol: 4-CIP, and 2-chlorophenol: 2-CIP) onto MWCNTs (MWCNT 8-15 nm in outer diameter (OD): MWCNT > $50 \mathrm{~nm}$ OD, helical MWCNT 100-200 nm OD, MWCNT $-\mathrm{COOH}<8 \mathrm{~nm}$ OD, and MWCNT-COOH $>50 \mathrm{~nm}$ OD) via DFT method (Watkins et al., 2017). The MWCNT-COOH $<8$ nm OD has revealed the highest adsorption for all the investigated chlorophenols. The DFT results affirmed that the physisorption or noncovalent interaction between the chlorophenols benzene ring and CNTs was the principal sorption mechanism, while charge transfer from the functional groups was considered less effective in the adsorption mechanism. Moreover, five structure-property relationship models reliably defined features of the intramolecular interactions.

The use of carbon nanotube as adsorbents for the elimination of drugs and pesticides from aqueous solutions is summarized in Table 3.

\subsubsection{Carbon and graphene quantum dots}

Carbon quantum dots are tiny fluorescent carbon nanoparticles with dimensions of less than $10 \mathrm{~nm}$ in diameter, and graphene quantum dots are a subset of carbon quantum dots generally derived from graphene/graphene oxide. The graphene quantum dots are small graphene sheets with lateral dimensions less than $10 \mathrm{~nm}$ with less than 10 graphene layers (Facure et al., 2020). The carbon quantum dots and graphene quantum dots, known as zerodimensional carbon based nanomaterials, are synthesized by using bottom-up and top-down methods (Iravani and Varma, 2020). They are commonly composed of $\mathrm{H}, \mathrm{C}, \mathrm{N}$, and $\mathrm{O}$, of which $\mathrm{O}$ and $\mathrm{C}$ are the most abundant elements because of the $-\mathrm{COOH}$ functional group existence. The carbon quantum dots and graphene quantum dots with their eminent physical-chemical properties including potent chemical inertness, low toxicity, plenty of edge functionalization sites, and high surface areas have been attracting increasing attention in various fields such as sensing (M. Z. Li et al., 2019a,b), detection (Chu et al., 2020), extraction (Mohebbi et al., 2020), and membrane fabrication (Zhao and Chung, 2018), along with wastewater treatment via adsorption (Anand et al., 2020) and photocatalytic (Lee et al., 2020) techniques.

The graphene quantum dots were synthesized by using the microwave-assisted hydrothermal method, and applied as an adsorbent $\left(\mathrm{q}_{\max }=130.10 \mathrm{mg} \mathrm{g}^{-1}\right.$ ) for the removal of 'oxamyl' carbamate pesticide from aqueous solutions (Agarwal et al., 2016). The isotherm, kinetic, and thermodynamic of adsorption process were investigated for proposing the adsorption mechanism. The results revealed that the adsorption isotherm and kinetic data were well fitted with the Langmuir isotherm and pseudo-second-order kinetic model. The oxamyl adsorption was occurred via feasible, spontaneous and endothermic process, and the adsorption mechanism was comprised the ionic interactions and electrostatic interactions between graphene quantum dots and oxamyl cation.

Despite the outstanding properties of carbon quantum dots and graphene quantum dots for removal of organic contaminants from water, very few researches have been reported for drugs and pesticides adsorption. 

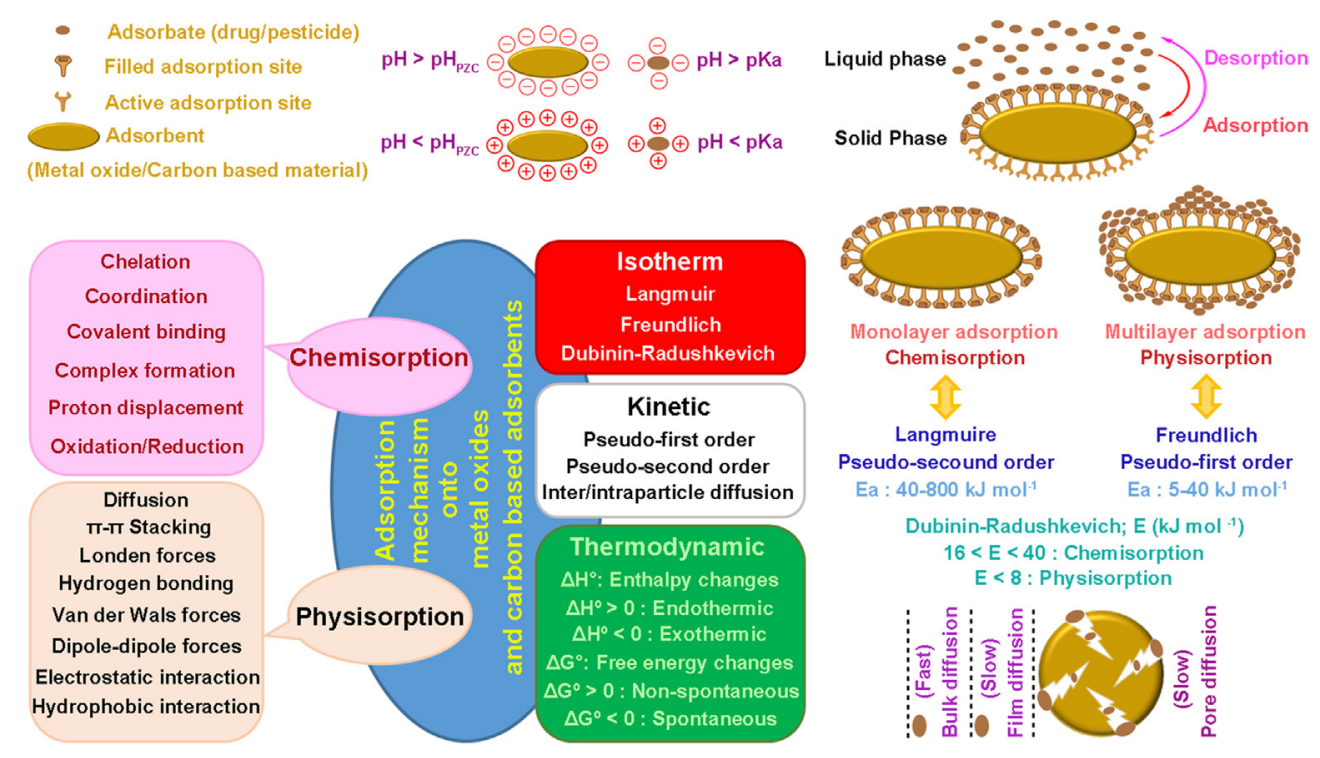

Fig. 14. Schematic illustration for the adsorption mechanism of drugs and pesticides onto metal oxides and carbon-based materials.

\section{The adsorption mechanism for drugs and pesticides}

Comprehending the adsorption mechanism is an important challenge and remarkable phase that can offer improvements in adsorption/desorption conditions or the design of adsorbent. The efficacy and mechanism of adsorption rely on the adsorbents' characteristics (e.g., surface area, porosity, and surface functional groups) (Tong et al., 2019), molecular properties of organic contaminants (e.g., molecular size, aliphatic vs. aromatic, and hydrophobicity) (Ai et al., 2019), background solution properties (ionic strength, $\mathrm{pH}$, natural organic matter, and temperature) (Xiang et al., 2019), and the interactions among functional groups of adsorbent and adsorbate (Kim et al., 2018). The adsorption mechanisms reported in the literature are exclusively either chemisorption or physisorption, based on the strength of interactions between sorbate and sorbent (Gusain et al., 2020). The physisorption occurs due to the reversible weak intermolecular physical interactions such as diffusion, $\pi-\pi$ stacking, hydrophobic interactions, London, dipoledipole and van der Waals forces, and hydrogen bonding with multilayer formation of adsorbate onto the adsorbent. While, the chemisorption involves the irreversible monolayer chemical interactions between the sorbate and sorbent by sharing or transfer of electrons, including covalent bonding, chelation, complex formation, proton displacement, and the redox reactions.

The solution $\mathrm{pH}$ is one of the important factors that control the adsorption process because the surface charges of adsorbent and adsorbate are managed by it. The adsorption regulation could be achieved by measuring the point of zero charges (PZC) of sorbents using the zeta potential (Fallah et al., 2020); $\mathrm{pH}_{\mathrm{PZC}}$ is a $\mathrm{pH}$ value at which the electrical charge density of a surface is zero.

At $\mathrm{pH}>\mathrm{pH}$ PZC, the functional groups are deprotonated and the adsorbent surface is negatively charged. Hence the sorbent successfully removes the cationic sorbates via electrostatic attraction from the aqueous solution. At $\mathrm{pH}<\mathrm{pH}_{\mathrm{PzC}}$, the protonation of functional groups leads to a positively charged adsorbent surface and the removal process is favorable for the anionic sorbates. If $\mathrm{pH}>\mathrm{pKa}$, the sorbate surface charge is negative; however, at $\mathrm{pH}<\mathrm{pKa}$, the sorbate exists in a positively charged surface. Thus, the adsorption capacity of the adsorbent can be altered by changing the solution $\mathrm{pH}$ concerning the adsorbent $\mathrm{pH}_{\mathrm{PZC}}$ and adsorbate $\mathrm{pKa}$ values.
The evaluation of adsorption efficiency using isotherm, kinetic models, and thermodynamic parameters is very effective in predicting the adsorption mechanism (Fallah et al., 2019). Considering the adsorption properties and equilibrium data of sorbent and sorbate, the adsorption isotherm models can define the interaction mechanism of sorbate onto the sorbent at a specific solution $\mathrm{pH}$, time, and temperature. To predict the multilayers or monolayer and heterogeneous or homogenous adsorption mechanism, several isotherm models including Freundlich (1906), Langmuir (Langmuir, 1917, 1916), Temkin (Temkin and Pyzhev, 1940), DubininRadushkevich (Dubinin and Radushkevich, 1947), Sips (1948), Redlich-Peterson (Redlich and Peterson, 1959), Jovanovich (Kudzinski and Wojciechowski, 1977), Flory-Huggins (Nikitas, 1984), and Khan (Khan et al., 1997), have been extended to fit experimental results. However, the Freundlich, Langmuir, and Dubinin Radushkevich models are commonly used for evaluating the isotherm studies. The Langmuir model is applied for computing the adsorbent maximum adsorption capacity $\left(\mathrm{q}_{\max } ; \mathrm{mg} \mathrm{g}^{-1}\right)$ and illustrates monolayer adsorption onto the homogenous sorbent surface, whereas the Freundlich model reveals the multilayer sorption onto the heterogeneous adsorbent surface. The DubininRadushkevich isotherm estimates the mean free adsorption energy of adsorbent $\left(\mathrm{E}, \mathrm{J} \mathrm{mol}^{-1}\right)$, where the $\mathrm{E}$ value smaller than 8 is suggesting the physisorption, $8<\mathrm{E}<16$ corresponding to ion exchange, and $16<\mathrm{E}<40$ indicating a chemisorption mechanism.

The kinetic models are investigated to explore the adsorption rate and adsorption rate-determining step at a specific solution $\mathrm{pH}$, concentration, and temperature. To determine the order of the adsorption process, diverse kinetic models have been suggested, including inter/intra-particle diffusion (Weber and Morris, 1963), pseudo-first-order (Ho, 2004), pseudo-second-order (Ho, 2006; Ho and McKay, 1999), and Elovich (Wu et al., 2009) models. The kinetic study of the adsorption process is typically evaluated by using the pseudo-first-order, pseudo-second-order, and intra-particle diffusion models. The physical interactions of adsorption at the primary stage are explained by the pseudo-first-order kinetic model, whereas the chemisorption rate-determining step of an adsorption process is determined by the pseudo-second-order kinetic model (Ahmed and Hameed, 2019). The diffusion process occurs in three consecutive steps: I) bulk diffusion, II) film diffusion: transportation from the bulk solution to the sorbent surface across the boundary 
layer by diffusion, and III) pore diffusion (intra-particle diffusion): diffusion from the outer surface into the adsorbent pores. The film diffusion and pore diffusion steps are mainly controlled by the diffusion process with slow speed (L. Wang et al., 2020).

The thermodynamic parameters, e.g., free energy changes $\left(\Delta \mathrm{G}^{\circ}\right)$, standard enthalpy changes $\left(\Delta \mathrm{H}^{\circ}\right)$, and standard entropy changes $\left(\Delta S^{\circ}\right)$, play a crucial role in determining the spontaneity and favorability of the sorption process, which can be calculated at a constant concentration, time and different temperatures (Fallah et al., 2018). The $\Delta G^{\circ}$ is determined from $\Delta S^{\circ}$ and $\Delta H^{\circ}$ values obtained by Van't Hoff plot. The adsorption process occurs spontaneously when $\Delta G^{\circ}$ is a negative value. If $\Delta H^{\circ}$ is a negative value, the adsorption reaction occurs in exothermic form, and if $\Delta \mathrm{H}^{\circ}$ is a positive value, the adsorption reaction will be endothermic. The adsorption affinity of sorbate onto the sorbent is revealed by the positive value of $\Delta \mathrm{S}^{\circ}$, which can suggest the increase of randomness.

The activation energy $\left(\mathrm{E}_{\mathrm{a}} ; \mathrm{kJ} \mathrm{mol}^{-1}\right)$, computed by the Arrhenius equation, provides the adsorption rate and the chemical or physical nature of adsorption. The $\mathrm{E}_{\mathrm{a}}$ values of physical and chemical adsorptions are usually from 5 to 40 to $40-800 \mathrm{~kJ} \mathrm{~mol}^{-1}$, respectively (Fallah et al., 2019). The chemisorption with higher activation energy and lower adsorption rate leads to efficient and strong interactions between sorbate and sorbent active sites.

Therefore, isotherms and kinetics models, along with thermodynamic parameters and activation energy, can clarify the physisorption or chemisorption characteristics of an adsorption process. Different methods of drugs or pesticide adsorption on metal oxide adsorbents or their composites and the adsorption mechanisms are schematically presented in Fig. 14.

\section{Conclusion and outlook}

In this article, a discussion on the toxicological effects of pharmaceutical drugs and pesticides on human life and ecology has been expounded. The residual drugs in the human body cause various serious issues such as genetic disorders, sickness, and cell death, and ecological drug contamination affects wildlife. The presence of pesticides in the body leads to Parkinson's disease through disruption of mitochondrial function, and they are even carcinogenic. Subsequently, we have discussed the recent advances in drug and pesticide removal via adsorption processes using various metal oxides and carbon-based nanostructures. Metal oxides have been employed as effective adsorbents due to their low toxicity, thermal stability, high surface area, porous structure, easy recovery, and the presence of Lewis acid-base sites in their structures. Besides, the utilization of assorted carbon-based materials such as activated carbon, graphene and its various derivatives, carbon nanotubes, and carbon/graphene quantum dots for water detoxification has also been presented in detail. They exhibit some extraordinary characteristics, including high thermal and mechanical stability, corrosion resistance, large surface area, high reactivity, hardness, and low density. Finally, the mechanism of adsorption of drugs and pesticides on the adsorbent surface has also been discussed, which is dependent upon adsorbents characteristics, molecular properties of drugs and pesticides, solution properties, and the type of interactions between functional groups of adsorbent and adsorbate. Thus, the presented review provides a critical study of the toxicological effect of drugs and pesticides and their probable removal from water bodies using metal oxides and carbon nanostructures via adsorption and hopefully stimulate further sustainable exploitation of earth-abundant materials in the future.

\section{Disclaimer}

The research presented was not performed or funded by EPA and was not subject to EPA's quality system requirements. The views expressed in this article are those of the author(s) and do not necessarily represent the views or the policies of the U.S. Environmental Protection Agency.

\section{Declaration of competing interest}

The authors declare that they have no known competing financial interests or personal relationships that could have appeared to influence the work reported in this paper.

\section{Appendix A. Supplementary data}

Supplementary data to this article can be found online at https://doi.org/10.1016/j.chemosphere.2021.130055.

\section{References}

Abbas, R.F., Hami, H.K., Mahdi, N.I., 2019. Removal of doxycycline hyclate by adsorption onto cobalt oxide at three different temperatures: isotherm, thermodynamic and error analysis. Int. J. Environ. Sci. Technol. 16, 5439-5446.

Abdelillah Ali Elhussein, E., Şahin, S., Bayazit, Ş.S., 2018. Preparation of $\mathrm{CeO}_{2}$ nanofibers derived from Ce-BTC metal-organic frameworks and its application on pesticide adsorption. J. Mol. Liq. 255, 10-17.

Abo El Naga, A.O., El Saied, M., Shaban, S.A., El Kady, F.Y., 2019. Fast removal of diclofenac sodium from aqueous solution using sugar cane bagasse-derived activated carbon. J. Mol. Liq. 285, 9-19.

Agarwal, S., Sadeghi, N., Tyagi, I., Gupta, V.K., Fakhri, A., 2016. Adsorption of toxic carbamate pesticide oxamyl from liquid phase by newly synthesized and characterized graphene quantum dots nanomaterials. J. Colloid Interface Sci. $478,430-438$.

Aguinaga Martínez, M.V., Llamas, N.E., Ávila Orozco, F.D., Domini, C.E., Acebal, C.C., 2020. Oxidized carbon nanotubes as sorbent for miniaturized solid-phase extraction of progestins from environmental water samples prior to their determination by HPLC-UV. Microchim. Acta 187, 153.

Ahmad, J., Naeem, S., Ahmad, M., Usman, A.R.A., Al-Wabel, M.I., 2019. A critical review on organic micropollutants contamination in wastewater and removal through carbon nanotubes. J. Environ. Manag. 246, 214-228.

Ahmad, K.S., 2019. Adsorption evaluation of herbicide iodosulfuron followed by cedrus deodora sawdust-derived activated carbon removal. Soil Sediment Contam. An Int. J. 28, 65-80.

Ahmad, T., Rafatullah, M., Ghazali, A., Sulaiman, O., Hashim, R., Ahmad, A., 2010. Removal of pesticides from water and wastewater by different adsorbents: a review. J. Environ. Sci. Heal. Part C 28, 231-271.

Ahmadijokani, F., Ahmadipouya, S., Molavi, H., Rezakazemi, M., Aminabhavi, T.M., Arjmand, M., 2020a. Impact of scale, activation solvents, and aged conditions on gas adsorption properties of UiO-66. J. Environ. Manag. 274, 111155.

Ahmadijokani, F., Mohammadkhani, R., Ahmadipouya, S., Shokrgozar, A., Rezakazem, M., Molavi, H., Aminabhavi, T.M., Arjmand, M., 2020b. Superior chemical stability of UiO-66 metal-organic frameworks (MOFs) for selective dye adsorption. Chem. Eng. J. 125346.

Ahmadijokani, F., Tajahmadi, S., Bahi, A., Molavi, H., Rezakazemi, M., Ko, F. Aminabhavi, T.M., Arjmand, M., 2020c. Ethylenediamine-functionalized Zrbased MOF for efficient removal of heavy metal ions from water. Chemosphere 128466.

Ahmed, M.B., Zhou, J.L., Ngo, H.H., Guo, W., Thomaidis, N.S., Xu, J., 2017. Progress in the biological and chemical treatment technologies for emerging contaminant removal from wastewater: a critical review. J. Hazard Mater. 323, 274-298.

Ahmed, M.J., 2017. Adsorption of non-steroidal anti-inflammatory drugs from aqueous solution using activated carbons: Review. J. Environ. Manag. 190, $274-282$.

Ahmed, M.J., Hameed, B.H., 2019. Insights into the isotherm and kinetic models for the coadsorption of pharmaceuticals in the absence and presence of metal ions: a review. J. Environ. Manag. 252, 109617.

Ai, Y., Liu, Y., Huo, Y., Zhao, C., Sun, L., Han, B., Cao, X., Wang, X., 2019. Insights into the adsorption mechanism and dynamic behavior of tetracycline antibiotics on reduced graphene oxide (RGO) and graphene oxide (GO) materials. Environ. Sci. Nano 6, 3336-3348.

Akhond, M., Absalan, G., Rafatmah, E., 2016. Studying the adsorption process of riboflavin on silver-deposited $\mathrm{Fe}_{3} \mathrm{O}_{4}$ nanoparticles. Anal. Bioanal. Chem. Res. 3, 225-237.

Al-Gubory, K.H., 2014. Environmental pollutants and lifestyle factors induce oxidative stress and poor prenatal development. Reprod. Biomed. Online 29, $17-31$.

Al-Jabari, M.H., Sulaiman, S., Ali, S., Barakat, R., Mubarak, A., Khan, S.A., 2019. 
Adsorption study of levofloxacin on reusable magnetic nanoparticles: kinetics and antibacterial activity. J. Mol. Liq. 291, 111249.

Al-Shaalan, N.H., Ali, I., ALOthman, Z.A., Al-Wahaibi, L.H., Alabdulmonem, H., 2019. High performance removal and simulation studies of diuron pesticide in water on MWCNTs. J. Mol. Liq. 289, 111039.

Alahabadi, A., Hosseini-Bandegharaei, A., Moussavi, G., Amin, B., Rastegar, A., Karimi-Sani, H., Fattahi, M., Miri, M., 2017. Comparing adsorption properties of $\mathrm{NH}_{4} \mathrm{Cl}$-modified activated carbon towards chlortetracycline antibiotic with those of commercial activated carbon. J. Mol. Liq. 232, 367-381.

Ali, I., Alharbi, O.M.L., ALOthman, Z.A., Al-Mohaimeed, A.M., Alwarthan, A., 2019a. Modeling of fenuron pesticide adsorption on CNTs for mechanistic insight and removal in water. Environ. Res. 170, 389-397.

Ali, I., Alharbi, O.M.L., Tkachev, A., Galunin, E., Burakov, A., Grachev, V.A., 2018. Water treatment by new-generation graphene materials: hope for bright future. Environ. Sci. Pollut. Res. 25, 7315-7329.

Ali, I., Basheer, A.A., Mbianda, X.Y., Burakov, A., Galunin, E., Burakova, I., Mkrtchyan, E., Tkachev, A., Grachev, V., 2019b. Graphene based adsorbents for remediation of noxious pollutants from wastewater. Environ. Int. 127, 160-180.

ALOthman, Z.A., Badjah, A.Y., Ali, I., 2019. Facile synthesis and characterization of multi walled carbon nanotubes for fast and effective removal of 4-tert-octylphenol endocrine disruptor in water. J. Mol. Liq. 275, 41-48.

Alves, A.A. de A., da Luz, A.D., Tones, A.R.M., Nonato, T.C.M., Ruiz, G.L. de O., Sens, M.L., 2018. Removal of carbamate insecticides from drinking water through a fixed bed column of granular activated carbon: a thermodynamic, kinetic and equilibrium study of multicomponent adsorption. Desalin. Water Treat. 108, 171-182.

Amiri, M.J., Roohi, R., Arshadi, M., Abbaspourrad, A., 2020. 2,4-D adsorption from agricultural subsurface drainage by canola stalk-derived activated carbon: insight into the adsorption kinetics models under batch and column conditions. Environ. Sci. Pollut. Res. 27, 16983-16997.

Anand, K.V., Subala, A.S., Sumathi, K.S., Merin, S.A.L., 2020. A review on the removal of dye, pesticide and pathogens from waste water using quantum dots. Eur. J. Adv. Chem. Res. 1, 1-6.

Angın, D., Günes, S., 2020. The usage of orange pulp activated carbon in the adsorption of 2,4-dichlorophenoxy acetic acid from aqueous solutions. Int. J. Phytoremediation 1-9.

aus der Beek, T., Weber, F., Bergmann, A., Hickmann, S., Ebert, I., Hein, A., Küster, A., 2016. Pharmaceuticals in the environment-global occurrences and perspectives. Environ. Toxicol. Chem. 35, 823-835.

Aydin, S., Aydin, M.E., Beduk, F., Ulvi, A., 2019. Removal of antibiotics from aqueous solution by using magnetic $\mathrm{Fe}_{3} \mathrm{O}_{4} /$ red mud-nanoparticles. Sci. Total Environ. 670, 539-546.

Bagbi, Y., Sarswat, A., Mohan, D., Pandey, A., Solanki, P.R., 2017. Lead and chromium adsorption from water using L-cysteine functionalized magnetite $\left(\mathrm{Fe}_{3} \mathrm{O}_{4}\right)$ nanoparticles. Sci. Rep. 7, 7672

Bahrami, M., Amiri, M.J., Beigzadeh, B., 2018. Adsorption of 2,4dichlorophenoxyacetic acid using rice husk biochar, granular activated carbon, and multi-walled carbon nanotubes in a fixed bed column system. Water Sci. Technol. 78, 1812-1821.

Balasubramani, K., Sivarajasekar, N., Naushad, M., 2020. Effective adsorption of antidiabetic pharmaceutical (metformin) from aqueous medium using graphene oxide nanoparticles: equilibrium and statistical modelling. J. Mol. Liq. 301,112426 .

Banerjee, B.D., Seth, V., Ahmed, R.S., 2001. Pesticide-induced oxidative stress: perspective and trends. Rev. Environ. Health 16, 1-40.

Batzill, M., 2011. Fundamental aspects of surface engineering of transition metal oxide photocatalysts. Energy Environ. Sci. 4, 3275-3286.

Bayat, M., Alighardashi, A., Sadeghasadi, A., 2018. Fixed-bed column and batch reactors performance in removal of diazinon pesticide from aqueous solutions by using walnut shell-modified activated carbon. Environ. Technol. Innov. 12, 148-159.

Bedia, J., Belver, C., Ponce, S., Rodriguez, J., Rodriguez, J.J., 2018. Adsorption of antipyrine by activated carbons from $\mathrm{FeCl}_{3}$-activation of Tara gum. Chem. Eng. J. 333, 58-65.

Benzigar, M.R., Talapaneni, S.N., Joseph, S., Ramadass, K., Singh, G., Scaranto, J., Ravon, U., Al-Bahily, K., Vinu, A., 2018. Recent advances in functionalized micro and mesoporous carbon materials: synthesis and applications. Chem. Soc. Rev. 47, 2680-2721.

Bernal, V., Giraldo, L., Moreno-Piraján, J.C., 2020a. Thermodynamic study of triclosan adsorption from aqueous solutions on activated carbon. J. Therm. Anal. Calorim. 139, 913-921.

Bernal, V., Giraldo, L., Moreno-Piraján, J.C., 2020b. Insight into adsorbate-adsorbent interactions between aromatic pharmaceutical compounds and activated carbon: equilibrium isotherms and thermodynamic analysis. Adsorption 26, $153-163$.

Bernal, V., Giraldo, L., Moreno-Piraján, J.C., 2020c. Thermodynamic analysis of acetaminophen and salicylic acid adsorption onto granular activated carbon: importance of chemical surface and effect of ionic strength. Thermochim. Acta $683,178467$.

Bhateria, R., Singh, R., 2019. A review on nanotechnological application of magnetic iron oxides for heavy metal removal. J. Water Process Eng. 31, 100845.

Bhatnagar, A., Hogland, W., Marques, M., Sillanpää, M., 2013. An overview of the modification methods of activated carbon for its water treatment applications. Chem. Eng. J. 219, 499-511.

Bhattacharyya, S., Das, P., Datta, S., 2019. In: Ghosh, S.K. (Ed.), Removal of Ranitidine from Pharmaceutical Waste Water Using Activated Carbon (AC) Prepared from Waste Lemon Peel BT - Waste Water Recycling and Management. Springer Singapore, Singapore, pp. 123-141.

Bi, Z., Kong, Q., Cao, Y., Sun, G., Su, F., Wei, X., Li, X., Ahmad, A., Xie, L., Chen, C.M., 2019. Biomass-derived porous carbon materials with different dimensions for supercapacitor electrodes: a review. J. Mater. Chem. 7, 16028-16045.

Bianchi, E., Lessing, G., Brina, K.R., Angeli, L., Andriguetti, N.B., Peruzzo, J.R.S., do Nascimento, C.A., Spilki, F.R., Ziulkoski, A.L., da Silva, L.B., 2017. Monitoring the genotoxic and cytotoxic potential and the presence of pesticides and hydrocarbons in water of the Sinos River basin, southern Brazil. Arch. Environ. Contam. Toxicol. 72, 321-334.

Boxall, A.B.A., Rudd, M.A., Brooks, B.W., Caldwell, D.J., Choi, K., Hickmann, S. Innes, E., Ostapyk, K., Staveley, J.P., Verslycke, T., 2012. Pharmaceuticals and personal care products in the environment: what are the big questions? Environ. Health Perspect. 120, 1221-1229.

Brito, G.M., Roldi, L.L., Schetino, M.Â., Checon Freitas, J.C., Cabral Coelho, E.R., 2020. High-performance of activated biocarbon based on agricultural biomass waste applied for 2,4-D herbicide removing from water: adsorption, kinetic and thermodynamic assessments. J. Environ. Sci. Heal. Part B 55, 767-782.

Cai, W., Weng, X., Chen, Z., 2019. Highly efficient removal of antibiotic rifampicin from aqueous solution using green synthesis of recyclable nano- $\mathrm{Fe}_{3} \mathrm{O}_{4}$. Environ. Pollut. 247, 839-846.

Çalıșkan Salihi, E., Wang, J., Kabacaoğlu, G., Kırkulak, S., Šiller, L., 2020. Graphene oxide as a new generation adsorbent for the removal of antibiotics from waters. Separ. Sci. Technol. 1-9.

Carboneras, M.B., Villaseñor, J., Fernández-Morales, F.J., Rodrigo, M.A., Cañizares, P., 2018. Biological treatment of wastewater polluted with an oxyfluorfen-based commercial herbicide. Chemosphere 213, 244-251.

Carrales-Alvarado, D.H., Leyva-Ramos, R., Rodríguez-Ramos, I., MendozaMendoza, E., Moral-Rodríguez, A.E., 2020. Adsorption capacity of different types of carbon nanotubes towards metronidazole and dimetridazole antibiotics from aqueous solutions: effect of morphology and surface chemistry. Environ. Sci. Pollut. Res. 27, 17123-17137.

Cheng, W., Kalahroodi, E.L., Marsac, R., Hanna, K., 2019. Adsorption of quinolone antibiotics to goethite under seawater conditions: application of a surface complexation model. Environ. Sci. Technol. 53, 1130-1138.

Chowdhury, S., Pan, S., Balasubramanian, R., Das, P., 2019. Date Palm Based Activated Carbon for the Efficient Removal of Organic Dyes from Aqueous Environment BT - Sustainable Agriculture Reviews 34: Date Palm for Food, Medicine and the Environment, pp. 247-263.

Chu, H.W., Unnikrishnan, B., Anand, A. Lin, Y.W., Huang C.C., 2020. Carbon quantum dots for the detection of antibiotics and pesticides. J. Food Drug Anal. 28, 539-557.

Ciğeroğlu, Z., Özdemir, O.K., Sahin, S., Hașimoğlu, A., 2020. Naproxen adsorption onto graphene oxide nanopowders: equilibrium, kinetic, and thermodynamic studies. Water, Air, Soil Pollut. 231, 101.

Cimirro, N.F.G.M., Lima, E.C., Cunha, M.R., Dias, S.L.P., Thue, P.S., Mazzocato, A.C. Dotto, G.L., Gelesky, M.A., Pavan, F.A., 2020. Removal of pharmaceutical compounds from aqueous solution by novel activated carbon synthesized from lovegrass (Poaceae). Environ. Sci. Pollut. Res. 27, 21442-21454.

Crini, G., Lichtfouse, E., Wilson, L.D., Morin-Crini, N., 2018. Adsorption-Oriented Processes Using Conventional and Non-conventional Adsorbents for Wastewater Treatment, vol. 18, pp. 23-71.

Cunha, D.L., Mendes, M.P., Marques, M., 2019. Environmental risk assessment of psychoactive drugs in the aquatic environment. Environ. Sci. Pollut. Res. 26, 78-90.

Dąbrowski, A., 2001. Adsorption—from theory to practice. Adv. Colloid Interface Sci. 93, 135-224.

Danish, M., Ahmad, T., 2018. A review on utilization of wood biomass as a sustainable precursor for activated carbon production and application. Renew. Sustain. Energy Rev. 87, 1-21.

Das, R., Leo, B.F., Murphy, F., 2018. The toxic truth about carbon nanotubes in water purification: a perspective view. Nanoscale Res. Lett. 13, 1-10.

D’Cruz, B., Madkour, M., Amin, M.O., Al-Hetlani, E., 2020. Efficient and recoverable magnetic $\mathrm{AC}-\mathrm{Fe}_{3} \mathrm{O}_{4}$ nanocomposite for rapid removal of promazine from wastewater. Mater. Chem. Phys. 240, 122109.

de Camargo Lima Beluci, N., Tonial dos Santos, T.R., Marcuzzo, J.S., Bergamasco, R. 2021. Facile filtration system to remove Diuron in aqueous solutions. J. Hazard Mater. 404, 124163.

de Franco, M.A.E., de Carvalho, C.B., Bonetto, M.M., de Pelegrini Soares, R., Féris, L.A. 2018. Diclofenac removal from water by adsorption using activated carbon in batch mode and fixed-bed column: isotherms, thermodynamic study and breakthrough curves modeling. J. Clean. Prod. 181, 145-154.

De Groot, M.J., 2006. Designing better drugs: predicting cytochrome P450 metabolism. Drug Discov. Today 11, 601-606.

de Souza Antônio, R., Guerra, A.C.S., de Andrade, M.B., Nishi, L., Baptista, A.T.A. Bergamasco, R., Vieira, A.M.S., 2021. Application of graphene nanosheet oxide for atrazine adsorption in aqueous solution: synthesis, material characterization, and comprehension of the adsorption mechanism. Environ. Sci. Pollut. Res. 28, 5731-5741.

de Souza, R.B., Moreira-de-Sousa, C., Ansoar-Rodríguez, Y., Coelho, M.P.M., de Souza, C.P., Bueno, O.C., Fontanetti, C.S., 2019. Histopatology and HSP70 analysis of the midgut of Rhinocricus padbergi (Diplopoda) in the evaluation of the toxicity of two new metallic-insecticides. Environ. Sci. Pollut. Res. 27, 3023-3033. 
Dehaghi, S.M., Rahmanifar, B., Moradi, A.M., Azar, P.A., 2014. Removal of permethrin pesticide from water by chitosan-zinc oxide nanoparticles composite as an adsorbent. J. Saudi Chem. Soc. 18, 348-355.

Dehghani, M.H., Kamalian, S., Shayeghi, M., Yousefi, M., Heidarinejad, Z., Agarwal, S., Gupta, V.K., 2019. High-performance removal of diazinon pesticide from water using multi-walled carbon nanotubes. Microchem. J. 145, 486-491.

Dehghani, M.H., Niasar, Z.S., Mehrnia, M.R., Shayeghi, M., Al-Ghouti, M.A., Heibati, B., McKay, G., Yetilmezsoy, K., 2017. Optimizing the removal of organophosphorus pesticide malathion from water using multi-walled carbon nanotubes. Chem. Eng. J. 310, 22-32.

del Mar Orta, M., Martín, J., Medina-Carrasco, S., Santos, J.L., Aparicio, I., Alonso, E., 2019. Adsorption of propranolol onto montmorillonite: kinetic, isotherm and pH studies. Appl. Clay Sci. 173, 107-114.

Del Vecchio, P., Haro, N.K., Souza, F.S., Marcílio, N.R., Féris, L.A., 2019. Ampicillin removal by adsorption onto activated carbon: kinetics, equilibrium and thermodynamics. Water Sci. Technol. 79, 2013-2021.

Deokar, S.K., Bajad, G.S., Bhonde, P., Vijayakumar, R.P., Mandavgane, S.A., 2017. Adsorptive removal of diuron herbicide on carbon nanotubes synthesized from plastic waste. J. Polym. Environ. 25, 165-175.

Derylo-Marczewska, A., Blachnio, M., Marczewski, A.W., Swiatkowski, A., Buczek, B., 2017. Adsorption of chlorophenoxy pesticides on activated carbon with gradually removed external particle layers. Chem. Eng. J. 308, 408-418.

Dhiman, N., Sharma, N., 2019a. Batch adsorption studies on the removal of ciprofloxacin hydrochloride from aqueous solution using $\mathrm{ZnO}$ nanoparticles and groundnut (Arachis hypogaea) shell powder: a comparison. Indian Chem. Eng. $61,67-76$.

Dhiman, N., Sharma, N., 2019b. Removal of pharmaceutical drugs from binary mixtures by use of $\mathrm{ZnO}$ nanoparticles: (Competitive adsorption of drugs). Environ. Technol. Innov. 15, 100392.

Djurišić, A.B., Leung, Y.H., Ng, A.M.C., Xu, X.Y., Lee, P.K.H., Degger, N., Wu, R.S.S., 2015. Toxicity of metal oxide nanoparticles: mechanisms, characterization, and avoiding experimental artefacts. Small 11, 26-44.

Dubinin, M.M., Radushkevich, L.V., 1947. Equation of the characteristic curve of activated charcoal proceedings of the academy of sciences. Phys. Chem. Sect. USSR 55, 331-333.

Ellenbecker, M., Tsai, S.J., Jacobs, M., Riediker, M., Peters, T., Liou, S., Avila, A., FossHansen, S., 2018. The difficulties in establishing an occupational exposure limit for carbon nanotubes. J. Nanoparticle Res. 20, 1-12.

Erdem, H., Yildiz, B., Şahin, M., Erdem, M., 2020. Etodolac adsorption onto activated carbon prepared by chemical activation and pyrolysis of biomasses mixture. Biomass Convers, Biorefinery, 10, 1153-1165.

Ersan, G., Apul, O.G., Perreault, F., Karanfil, T., 2017. Adsorption of organic contaminants by graphene nanosheets: a review. Water Res. 126, 385-398.

Facure, M.H.M., Schneider, R., Mercante, L.A., Correa, D.S., 2020. A review on graphene quantum dots and their nanocomposites: from laboratory synthesis towards agricultural and environmental applications. Environ. Sci. Nano 7, 3710-3734.

Fakhri, A. Behrouz, S., 2015. Comparison studies of adsorption properties of $\mathrm{MgO}$ nanoparticles and $\mathrm{ZnO}-\mathrm{MgO}$ nanocomposites for linezolid antibiotic removal from aqueous solution using response surface methodology. Process Saf. Environ. Protect. 94, 37-43.

Falahian, Z., Torki, F., Faghihian, H., 2018. Synthesis and application of polypyrrole/ $\mathrm{Fe}_{3} \mathrm{O}_{4}$ nanosize magnetic adsorbent for efficient separation of $\mathrm{Hg}^{2+}$ from aqueous solution. Glob. Challenges 2, 1700078.

Fallah, Z., Nasr Isfahani, H., Tajbakhsh, M., 2020. Removal of fluoride ion from aqueous solutions by titania-grafted $\beta$-cyclodextrin nanocomposite. Environ. Sci. Pollut. Res. 27, 3281-3294.

Fallah, Z., Nasr Isfahani, H., Tajbakhsh, M., 2019. Cyclodextrin-triazole-titanium based nanocomposite: preparation, characterization and adsorption behavior investigation. Process Saf. Environ. Protect. 124, 251-265.

Fallah, Z., Nasr Isfahani, H., Tajbakhsh, M., Tashakkorian, H., Amouei, A., 2018. TiO 2 grafted cellulose via click reaction: an efficient heavy metal ions bioadsorbent from aqueous solutions. Cellulose 25, 639-660.

Freundlich, H., 1906. Uber die adsorption in losungen. Z. Phys. Chem. 57U, 385-470.

Gan, Q., Zhang, T., Chen, R., Wang, X., Ye, M., 2019. Simple, low-dose, durable, and carbon-nanotube-based floating solar still for efficient desalination and purification. ACS Sustain. Chem. Eng. 7, 3925-3932.

Gao, N., Yan, Y., 2012. Characterisation of surface wettability based on nanoparticles. Nanoscale 4, 2202-2218.

Gar Alalm, M., Nasr, M., 2018. Artificial intelligence, regression model, and cost estimation for removal of chlorothalonil pesticide by activated carbon prepared from casuarina charcoal. Sustain. Environ. Res. 28, 101-110.

Geczo, A., Giannakoudakis, D.A., Triantafyllidis, K., Elshaer, M.R., RodríguezAguado, E., Bashkova, S., 2020. Mechanistic insights into acetaminophen removal on cashew nut shell biomass-derived activated carbons. Environ. Sci. Pollut. Res. https://doi.org/10.1007/s11356-019-07562-0.

Gil, A., Santamaría, L., Korili, S.A., 2018. Removal of caffeine and diclofenac from aqueous solution by adsorption on multiwalled carbon nanotubes. Colloid Interface Sci. Commun. 22, 25-28.

Goli, A., Alinezhad, H., Ganji, M.D., 2020. Theoretical insights into the performance of graphene derivatives, h-BN and BNC heterostructures in the adsorption and elimination of atrazine: an all-electron DFT study. Diam. Relat. Mater. 108, 107967.

Goswami, L., Manikandan, N.A., Dolman, B., Pakshirajan, K., Pugazhenthi, G., 2018. Biological treatment of wastewater containing a mixture of polycyclic aromatic hydrocarbons using the oleaginous bacterium Rhodococcus opacus. J. Clean. Prod. 196, 1282-1291.

Grabarczyk, Ł., Mulkiewicz, E., Stolte, S., Puckowski, A., Pazda, M., Stepnowski, P., Białk-Bielińska, A., 2020. Ecotoxicity screening evaluation of selected pharmaceuticals and their transformation products towards various organisms. Environ. Sci. Pollut. Res. Int. 27, 26103-26114.

Grant, G.A., Fisher, P.R., Barrett, J.E., Wilson, P.C., 2019. Removal of agrichemicals from water using granular activated carbon filtration. Water, Air, Soil Pollut. 230, 7.

Grant, G.A., Fisher, P.R., Barrett, J.E., Wilson, P.C., 2018. Removal of paclobutrazol from irrigation water using granular-activated carbon. Irrigat. Sci. 36, 159-166.

Gray, J.W., Burns, C.J., Mahlburg, W.M., 2013. Increased cancer burden among pesticide applicators and others due to pesticide exposure. Ca - Cancer J. Clin. 63, 364-366.

Green, R.M., Hodges, N.J., Chipman, J.K., O’Donovan, M.R., Graham, M., 2008 Reactive oxygen species from the uncoupling of human cytochrome P450 1B1 may contribute to the carcinogenicity of dioxin-like polychlorinated biphenyls. Mutagenesis 23, 457-463.

Guerra, A.C.S., de Andrade, M.B., Tonial dos Santos, T.R., Bergamasco, R., 2019 Adsorption of sodium diclofenac in aqueous medium using graphene oxide nanosheets. Environ. Technol. 1-11.

Guo, Y., Tan, C., Sun, J., Li, W., Zhang, J., Zhao, C., 2020. Porous activated carbons derived from waste sugarcane bagasse for $\mathrm{CO}_{2}$ adsorption. Chem. Eng. J. 381, 122736.

Gupta, A.K., Naregalkar, R.R., Vaidya, V.D., Gupta, M., 2007. Recent Advances on Surface Engineering of Magnetic Iron Oxide Nanoparticles and Their Biomedica Applications, , 1st2. Future Medicine, pp. 23-39.

Gusain, R., Gupta, K., Joshi, P., Khatri, O.P., 2019. Adsorptive removal and photocatalytic degradation of organic pollutants using metal oxides and their composites: a comprehensive review. Adv. Colloid Interface Sci. 272, 102009.

Gusain, R., Kumar, N., Ray, S.S., 2020. Recent advances in carbon nanomaterialbased adsorbents for water purification. Coord. Chem. Rev. 405, 213111.

Hallenbeck, W.H., Cunningham-Burns, K.M., 2012. Pesticides and Human Health, first ed. Springer Science \& Business Media.

Han, X., Meng, L., Li, Y., Li, A., Turyk, M.E., Yang, R., Wang, P., Xiao, K., Zhao, J. Zhang, J., 2020. Associations between the exposure to persistent organic pollutants and type 2 diabetes in East China: a case-control study. Chemosphere $241,125030$.

Haro, N.K., Del Vecchio, P., Marcilio, N.R., Féris, L.A., 2017. Removal of atenolol by adsorption - study of kinetics and equilibrium. J. Clean. Prod. 154, 214-219.

Hartz, K.E.H. Nutile, S.A, Fung, C.Y Sinche, F.L, Moran, P.W Van Metre, P.C. Nowell, L.H., Lydy, M.J., 2019. Survey of bioaccessible pyrethroid insecticides and sediment toxicity in urban streams of the northeast United States. Environ. Pollut. 254, 112931.

Hasanzadeh, V., Rahmanian, O., Heidari, M., 2020. Cefixime adsorption onto activated carbon prepared by dry thermochemical activation of date fruit residues. Microchem. J. 152, 104261.

Hashemi, M.S., Gharbi, S., Jafarinejad-Farsangi, S., Ansari-Asl, Z., Dezfuli, A.S., 2020 Secondary toxic effect of graphene oxide and graphene quantum dots alters the expression of miR-21 and miR-29a in human cell lines. Toxicol. Vitro 65 104796.

Heller, D.A., Jena, P.V., Pasquali, M., Kostarelos, K., Delogu, L.G., Meidl, R.E., Rotkin, S.V., Scheinberg, D.A., Schwartz, R.E., Terrones, M., 2020. Banning carbon nanotubes would be scientifically unjustified and damaging to innovation. Nat. Nanotechnol. 15, 164-166.

Hgeig, A., Novaković, M., Mihajlović, I., 2019. Sorption of carbendazim and linuron from aqueous solutions with activated carbon produced from spent coffee grounds: equilibrium, kinetic and thermodynamic approach. J. Environ. Sci. Heal. Part B 54, 226-236.

Ho, Y.S., 2006. Review of second-order models for adsorption systems. J. Hazard Mater. 136, 681-689.

Ho, Y.S., 2004. Citation review of Lagergren kinetic rate equation on adsorption reactions. Scientometrics 59, 171-177.

Ho, Y.S., McKay, G., 1999. Pseudo-second order model for sorption processes. Process Biochem. 34, 451-465.

Hu, C., Lin, Y., Connell, J.W., Cheng, H.M., Gogotsi, Y., Titirici, M.M., Dai, L., 2019 Carbon-based metal-free catalysts for energy storage and environmental remediation. Adv. Mater. 31, 1806128.

Hubetska, T., Kobylinska, N., García, J.R., 2020. Efficient adsorption of pharmaceutical drugs from aqueous solution using a mesoporous activated carbon. Adsorption 26, 251-266.

Hue, H.K., Anh, L.V., Thiep, T. Van, 2018. Study of the adsorption of 2,4dichlorophenoxyacetic acid from the aqueous solution onto carbon nanotubes. Vietnam J. Chem. 56, 191-196.

Iijima, S., 1991. Helical microtubules of graphitic carbon. Nature 354, 56-58.

Iravani, S., Varma, R.S., 2020. Green synthesis, biomedical and biotechnological applications of carbon and graphene quantum dots. A review. Environ. Chem. Lett. 18, 703-727.

Ismail, N.H., Salleh, W.N.W., Ismail, A.F., Hasbullah, H., Yusof, N., Aziz, F., Jaafar, J., 2020. Hydrophilic polymer-based membrane for oily wastewater treatment: a review. Separ. Purif. Technol. 233, 116007.

Jaladanki, C.K., Gahlawat, A., Rathod, G., Sandhu, H., Jahan, K., Bharatam, P.V., 2020. Mechanistic studies on the drug metabolism and toxicity originating from cytochromes P450. Drug Metab. Rev. 52, 366-394.

Johnson, A.C., Jürgens, M.D., Williams, R.J., Kümmerer, K., Kortenkamp, A. 
Sumpter, J.P., 2008. Do cytotoxic chemotherapy drugs discharged into rivers pose a risk to the environment and human health? An overview and UK case study. J. Hydrol. 348, 167-175.

Jung, C., Son, A., Her, N., Zoh, K.D., Cho, J., Yoon, Y., 2015. Removal of endocrine disrupting compounds, pharmaceuticals, and personal care products in water using carbon nanotubes: a review. J. Ind. Eng. Chem. 27, 1-11.

Kalumpha, M., Guyo, U., Zinyama, N.P., Vakira, F.M., Nyamunda, B.C., 2020. Adsorptive potential of Zea mays tassel activated carbon towards the removal of metformin hydrochloride from pharmaceutical effluent. Int. J. Phytoremediation 22, 148-156.

Kasperiski, F.M., Lima, E.C., Umpierres, C.S., dos Reis, G.S., Thue, P.S., Lima, D.R., Dias, S.L.P., Saucier, C., da Costa, J.B., 2018. Production of porous activated carbons from Caesalpinia ferrea seed pod wastes: highly efficient removal of captopril from aqueous solutions. J. Clean. Prod. 197, 919-929.

Kaur, H., Bansiwal, A., Hippargi, G., Pophali, G.R., 2018. Effect of hydrophobicity of pharmaceuticals and personal care products for adsorption on activated carbon: adsorption isotherms, kinetics and mechanism. Environ. Sci. Pollut. Res. 25, 20473-20485.

Kaur, Y., Bhatia, Y., Chaudhary, S., Chaudhary, G.R., 2017. Comparative performance of bare and functionalize $\mathrm{ZnO}$ nanoadsorbents for pesticide removal from aqueous solution. J. Mol. Liq. 234, 94-103.

Khalil, A.M.E., Memon, F.A., Tabish, T.A., Salmon, D., Zhang, S., Butler, D., 2020. Nanostructured porous graphene for efficient removal of emerging contaminants (pharmaceuticals) from water. Chem. Eng. J. 398, 125440.

Khan, A.R., Ataullah, R., Al-Haddad, A., 1997. Equilibrium adsorption studies of some aromatic pollutants from dilute aqueous solutions on activated carbon at different temperatures. J. Colloid Interface Sci. 194, 154-165.

Khoshnam, F., Zargar, B., Moghadam, M.R., 2019. Adsorption and removal of ametryn using graphene oxide nano-sheets from farm waste water and optimization using response surface methodology. J. Iran. Chem. Soc. 16, 1383-1390.

Kim, S., Park, C.M., Jang, M., Son, A., Her, N., Yu, M., Snyder, S., Kim, D.H., Yoon, Y., 2018. Aqueous removal of inorganic and organic contaminants by graphenebased nanoadsorbents: a review. Chemosphere 212, 1104-1124.

Kıdak, R., Doğan, S., 2018. Medium-high frequency ultrasound and ozone based advanced oxidation for amoxicillin removal in water. Ultrason. Sonochem. 40, $131-139$.

Kobayashi, N., Izumi, H., Morimoto, Y., 2017. Review of toxicity studies of carbon nanotubes. J. Occup. Health 59, 394-407.

Koe, W.S., Chong, W.C., Pang, Y.L., Koo, C.H., Ebrahim, M., Mohammad, A.W., 2020. Novel nitrogen and sulphur co-doped carbon quantum dots/titanium oxide photocatalytic membrane for in-situ degradation and removal of pharmaceutical compound. J. Water Process Eng. 33, 101068.

Kong, Q., He, X., Shu, L., Miao, M., 2017. Ofloxacin adsorption by activated carbon derived from luffa sponge: kinetic, isotherm, and thermodynamic analyses. Process Saf. Environ. Protect. 112, 254-264.

Koutník, I., Vráblová, M., Bednárek, J., 2020. Reynoutria japonica, an invasive herb as a source of activated carbon for the removal of xenobiotics from water. Bioresour. Technol. 309, 123315.

Kretschmer, X.C., Baldwin, W.S., 2005. CAR and PXR: xenosensors of endocrine disrupters? Chem. Biol. Interact. 155, 111-128.

Kudzinski, V., Wojciechowski, B.W., 1977. On the Jovanovic model of adsorption I. An extension for mobile adsorbed layers. Colloid Polym. Sci. 255, 869-880.

Kümmerer, K., 2008. Pharmaceuticals in the Environment: Sources, Fate, Effects and Risks. Springer Science \& Business Media.

Lalović, B., Đurkić, T., Vukčević, M., Janković-Častvan, I., Kalijadis, A., Laušević, Z., Laušević, M., 2017. Solid-phase extraction of multi-class pharmaceuticals from environmental water samples onto modified multi-walled carbon nanotubes followed by LC-MS/MS. Environ. Sci. Pollut. Res. 24, 20784-20793.

Länge, R., Dietrich, D., 2002. Environmental risk assessment of pharmaceutical drug substances-conceptual considerations. Toxicol. Lett. 131, 97-104.

Langmuir, I., 1917. The constitution and fundamental properties of solids and liquids. II. LIQUIDS.1. J. Am. Chem. Soc. 39, 1848-1906.

Langmuir, I., 1916. The constitution and fundamental properties of solids and liquids. Part I. Solids. J. Am. Chem. Soc. 38, 2221-2295.

Larsson, D.G.J., 2014. Pollution from drug manufacturing: review and perspectives. Philos. Trans. R. Soc. B Biol. Sci. 369, 20130571.

Lawal, I.A., Lawal, M.M., Akpotu, S.O., Okoro, H.K., Klink, M., Ndungu, P., 2020. Noncovalent graphene oxide functionalized with ionic liquid: theoretical, isotherm, kinetics, and regeneration studies on the adsorption of pharmaceuticals. Ind. Eng. Chem. Res. 59, 4945-4957.

Lazarević-Pašti, T., Anićijević, V., Baljozović, M., Anićijević, D.V., Gutić, S., Vasić, V., Skorodumova, N.V., Pašti, I.A., 2018. The impact of the structure of graphenebased materials on the removal of organophosphorus pesticides from water. Environ. Sci. Nano 5, 1482-1494.

Lee, D.H., Porta, M., Jacobs Jr., D.R., Vandenberg, L.N., 2014. Chlorinated persistent organic pollutants, obesity, and type 2 diabetes. Endocr. Rev. 35, 557-601.

Lee, H., Anwer, H., Park, J.W., 2020. Graphene quantum dots on stainless-steel nanotubes for enhanced photocatalytic degradation of phenanthrene under visible light. Chemosphere 246, 125761.

Li, M., Chen, T., Gooding, J.J., Liu, J., 2019a. Review of carbon and graphene quantum dots for sensing. ACS Sens. 4, 1732-1748.

Li, M., Liu, J., Xu, Y., Qian, G., 2016. Phosphate adsorption on metal oxides and metal hydroxides: a comparative review. Environ. Rev. 24, 319-332.

Li, Z., Wang, L., Li, Y., Feng, Y., Feng, W., 2019b. Carbon-based functional nanomaterials: preparation, properties and applications. Compos. Sci. Technol. 179,
$10-40$.

Liang, X., Fan, J., Liang, D., Xu, Y., Zhi, Y., Hu, H., Oiu, X., 2021. Surface hydroxyl groups functionalized graphite carbon nitride for high efficient removal of diquat dibromide from water. J. Colloid Interface Sci. 582, 70-80.

Lima, D.R., Hosseini-Bandegharaei, A., Thue, P.S., Lima, E.C., de Albuquerque, Y.R.T. dos Reis, G.S., Umpierres, C.S., Dias, S.L.P., Tran, H.N., 2019. Efficient acetaminophen removal from water and hospital effluents treatment by activated carbons derived from Brazil nutshells. Colloids Surfaces A Physicochem. Eng. Asp. $583,123966$.

Lin, J., Su, B., Sun, M., Chen, B., Chen, Z., 2018. Biosynthesized iron oxide nanoparticles used for optimized removal of cadmium with response surface methodology. Sci. Total Environ. 627, 314-321.

Liu, Y, Peng Y, An, B, Li, L, Liu, Y, 2020. Effect of molecular structure on the adsorption affinity of sulfonamides onto CNTs: batch experiments and DFT calculations. Chemosphere 246, 125778.

Lu, X., Zhu, Y., Bai, R., Wu, Z., Qian, W., Yang, L., Cai, R., Yan, H., Li, T., Pandey, V., 2019. Long-term pulmonary exposure to multi-walled carbon nanotubes promotes breast cancer metastatic cascades. Nat. Nanotechnol. 14, 719-727.

Ma, P., Liu, Q., Liu, P., Li, H., Han, X., Liu, L., Zou, W., 2020. Green synthesis of Fe/Cu oxides composite particles stabilized by pine needle extract and investigation of their adsorption activity for norfloxacin and ofloxacin. J. Dispersion Sci. Technol. $1-18$.

Macías-García, A., García-Sanz-Calcedo, J., Carrasco-Amador, J.P., Segura-Cruz, R. 2019. Adsorption of paracetamol in hospital wastewater through activated carbon filters. Sustainability 11, 2672.

Mahmoud, A.M., Ibrahim, F.A., Shaban, S.A., Youssef, N.A., 2015. Adsorption of heavy metal ion from aqueous solution by nickel oxide nano catalyst prepared by different methods. Egypt. J. Petrol. 24, 27-35.

Makvandi, P., Wang, C., Zare, E.N., Borzacchiello, A., Niu, L., Tay, F.R., 2020. Metalbased nanomaterials in biomedical applications: antimicrobial activity and cytotoxicity aspects. Adv. Funct. Mater. 30 https://doi.org/10.1002 adfm.201910021 (in press).

Malhotra, M., Suresh, S., Garg, A., 2018. Tea waste derived activated carbon for the adsorption of sodium diclofenac from wastewater: adsorbent characteristics, adsorption isotherms, kinetics, and thermodynamics. Environ. Sci. Pollut. Res. 25, 32210-32220.

Manjunath, S.V., Singh Baghel, R., Kumar, M., 2020. Antagonistic and synergistic analysis of antibiotic adsorption on Prosopis juliflora activated carbon in multicomponent systems. Chem. Eng. J. 381, 122713.

Mansour, F., Al-Hindi, M., Yahfoufi, R., Ayoub, G.M., Ahmad, M.N., 2018. The use of activated carbon for the removal of pharmaceuticals from aqueous solutions: a review. Rev. Environ. Sci. Bio/Technology 17, 109-145.

Mansouri, E.H., Reggabi, M., 2020. Association between type 2 diabetes and exposure to chlorinated persistent organic pollutants in Algeria: a case-control study. Chemosphere 128596.

Marican, A., Duran-Lara, E.F., 2018. A review on pesticide removal through different processes. Environ. Sci. Pollut. Res. Int. 25, 2051-2064.

Márquez, G., Rodríguez, E.M., Beltrán, F.J. Álvarez, P.M., 2014. Solar photocatalytic ozonation of a mixture of pharmaceutical compounds in water. Chemosphere $113,71-78$.

Menya, E., Olupot, P.W., Storz, H., Lubwama, M., Kiros, Y., 2018. Production and performance of activated carbon from rice husks for removal of natural organic matter from water: a review. Chem. Eng. Res. Des. 129, 271-296.

Milagres, J.L., Bellato, C.R., Ferreira, S.O., de Moura Guimarães, L., 2020. Preparation and evaluation of hydrocalumite-iron oxide magnetic intercalated with dodecyl sulfate for removal of agrichemicals. J. Environ. Manag. 255, 109845.

Misra, T., Mitra, S., Sen, S., 2018. Adsorption studies of carbamazepine by greensynthesized magnetic nanosorbents. Nanotechnol. Environ. Eng. 3, 11.

Mohammad, S.G., Ahmed, S.M., Amr, A.E.G.E., Kamel, A.H., 2020. Porous activated carbon from lignocellulosic agricultural waste for the removal of acetampirid pesticide from aqueous solutions. Molecules 25, 2339.

Mohebbi, A., Farajzadeh, M.A., Mahmoudzadeh, A., Etemady, A., 2020. Combination of poly ( $\varepsilon$-caprolactone) grafted graphene quantum dots-based dispersive solid phase extraction followed by dispersive liquid-liquid microextraction for extraction of some pesticides from fruit juices prior to their quantification by gas chromatography. Microchem. J. 153, 104328.

Molaei, M.J., 2019. A review on nanostructured carbon quantum dots and their applications in biotechnology, sensors, and chemiluminescence. Talanta 196, 456-478.

Moral-Rodríguez, A.I., Leyva-Ramos, R., Ania, C.O., Ocampo-Pérez, R., IsaacsPáez, E.D., Carrales-Alvarado, D.H., Parra, J.B., 2019. Tailoring the textura properties of an activated carbon for enhancing its adsorption capacity towards diclofenac from aqueous solution. Environ. Sci. Pollut. Res. 26, 6141-6152.

Moreira, V.R., Lebron, Y.A.R., da Silva, M.M., de Souza Santos, L.V., Jacob, R.S., de Vasconcelos, C.K.B., Viana, M.M., 2020. Graphene oxide in the remediation of norfloxacin from aqueous matrix: simultaneous adsorption and degradation process. Environ. Sci. Pollut. Res. 27, 34513-34528.

Nagpal, M., Kakkar, R., 2019. Use of metal oxides for the adsorptive removal of toxic organic pollutants. Separ. Purif. Technol. 211, 522-539.

Nasrollahzadeh, M., Sajjadi, M., Iravani, S., Varma, R.S., 2021a. Starch, cellulose, pectin, gum, alginate, chitin and chitosan derived (nano)materials for sustainable water treatment: a review. Carbohydr. Polym. 251, 116986.

Nasrollahzadeh, M., Sajjadi, M., Iravani, S., Varma, R.S., 2021b. Carbon-based sustainable nanomaterials for water treatment: state-of-art and future perspectives. Chemosphere 263, 128005. 
Nassar, M.Y., Ahmed, I.S., Hendy, H.S., 2018. A facile one-pot hydrothermal synthesis of hematite $\left(\alpha-\mathrm{Fe}_{2} \mathrm{O}_{3}\right)$ nanostructures and cephalexin antibiotic sorptive removal from polluted aqueous media. J. Mol. Liq. 271, 844-856.

Nayak, A., Bhushan, B., Sharma, P.K., Gupta, V., 2018. Development of magnetic nanoparticles from poplar sawdust for removal of pesticides from aqueous solution. J. Graph. Era Univ. 6, 55-70.

Nazal, M.K., 2020. An overview of carbon-based materials for the removal of pharmaceutical active compounds. Carbon-Based Mater. Environ. Prot. Remediat. 83.

Ncibi, M.C., Sillanpää, M., 2017. Optimizing the removal of pharmaceutical drugs Carbamazepine and Dorzolamide from aqueous solutions using mesoporous activated carbons and multi-walled carbon nanotubes. J. Mol. Liq. 238, 379-388.

Negara, D.N.K.P., Nindhia, T.G.T., Surata, I.W., Hidajat, F., Sucipta, M., 2019. Nanopore structures, surface morphology, and adsorption capacity of tabah bambooactivated carbons. Surfaces and Interfaces 16, 22-28.

Nekoueian, K., Amiri, M., Sillanpää, M., Marken, F., Boukherroub, R., Szunerits, S., 2019. Carbon-based quantum particles: an electroanalytical and biomedical perspective. Chem. Soc. Rev. 48, 4281-4316.

Nie, W., Li, Y., Chen, L., Zhao, Z., Zuo, X., Wang, D., Zhao, L., Feng, X., 2020. Interaction between multi-walled carbon nanotubes and propranolol. Sci. Rep. 10, 10259.

Nikitas, P., 1984. Generalized Flory-Huggins isotherms for adsorption from solution. J. Chem. Soc. Faraday Trans. 1 Phys. Chem. Condens. Phases 80, 3315-3329.

Nikolaou, A., Meric, S., Fatta, D., 2007. Occurrence patterns of pharmaceuticals in water and wastewater environments. Anal. Bioanal. Chem. 387, 1225-1234.

Nourmoradi, H., Moghadam, K.F., Jafari, A., Kamarehie, B., 2018. Removal of acetaminophen and ibuprofen from aqueous solutions by activated carbon derived from Quercus Brantii (Oak) acorn as a low-cost biosorbent. J. Environ. Chem. Eng. 6, 6807-6815.

Novoselov, K.S., Geim, A.K., Morozov, S.V., Jiang, D., Zhang, Y., Dubonos, S.V., Grigorieva, I.V., Firsov, A.A., 2004. Electric field effect in atomically thin carbon films. Science 306, 666-669.

Obodo, R.M., Ahmad, I., Ezema, F.I., 2019. Introductory chapter: graphene and its applications. In: Graphene and its Derivatives-Synthesis and Applications. IntechOpen, pp. 1-9.

Ogunleye, D.T., Akpotu, S.O., Moodley, B., 2020. Adsorption of sulfamethoxazole and reactive blue 19 using graphene oxide modified with imidazolium based ionic liquid. Environ. Technol. Innov. 17, 100616.

Onsori, S., Alipour, E., 2018. A theoretical investigation on the adsorption of platinol drug on a $\mathrm{ZnO}$ nanocluster: solvent and density functional effect. J. Mol. Liq. 256, 558-564.

Organization, W.H., 2012. Pharmaceuticals in Drinking-Water.

Pandiarajan, A., Kamaraj, R., Vasudevan, S., Vasudevan, S., 2018. OPAC (orange peel activated carbon) derived from waste orange peel for the adsorption of chlorophenoxyacetic acid herbicides from water: adsorption isotherm, kinetic modelling and thermodynamic studies. Bioresour. Technol. 261, 329-341.

Panic, S., Guzsvány, V., Kónya, Z., Kukovecz, Á., Boskovic, G., 2017. Kinetic, equilibrium and thermodynamic studies of thiamethoxam adsorption by multi-walled carbon nanotubes. Int. J. Environ. Sci. Technol. 14, 1297-1306.

Panwar, N., Soehartono, A.M., Chan, K.K., Zeng, S., Xu, G., Qu, J., Coquet, P., Yong, K.T., Chen, X., 2019. Nanocarbons for biology and medicine: sensing, imaging, and drug delivery. Chem. Rev. 119, 9559-9656.

Parashar, A., Sikarwar, S., Jain, R., 2020. Removal of drug oxcarbazepine from wastewater at 3D porous $\mathrm{NiFe}_{2} \mathrm{O}_{4}$ nanoparticles. J. Dispersion Sci. Technol. 41, 884-894.

Paredes-Laverde, M., Salamanca, M., Silva-Agredo, J., Manrique-Losada, L., TorresPalma, R.A., 2019. Selective removal of acetaminophen in urine with activated carbons from rice (Oryza sativa) and coffee (Coffea arabica) husk: effect of activating agent, activation temperature and analysis of physical-chemical interactions. J. Environ. Chem. Eng. 7, 103318.

Priyan, V.V., Shahnaz, T., Suganya, E., Sivaprakasam, S., Narayanasamy, S., 2020. Ecotoxicological assessment of micropollutant Diclofenac biosorption on magnetic sawdust: phyto, Microbial and Fish toxicity studies. J. Hazard Mater. 123532.

Qin, X., Du, P., Chen, J. Liu, F., Wang, G., Weng, L., 2018. Effects of natural organic matter with different properties on levofloxacin adsorption to goethite: experiments and modeling. Chem. Eng. J. 345, 425-431.

Radushkevich, L.V., Lukyanovich, V.M., 1952. Structure of the carbon produced in the thermal decomposition of carbon monoxide on an iron catalyst. Russ. J. Phys. Chem. 26, 88-95.

Rafati, L., Ehrampoush, M.H., Rafati, A.A., Mokhtari, M., Mahvi, A.H., 2018. Removal of ibuprofen from aqueous solution by functionalized strong nano-clay composite adsorbent: kinetic and equilibrium isotherm studies. Int. J. Environ. Sci. Technol. 15, 513-524.

Rahman, A., Hango, H.J., Daniel, L.S., Uahengo, V., Jaime, S.J., Bhaskaruni, S.V.H.S., Jonnalagadda, S.B., 2019a. Chemical preparation of activated carbon from Acacia erioloba seed pods using $\mathrm{H} 2 \mathrm{SO} 4$ as impregnating agent for water treatment: an environmentally benevolent approach. J. Clean. Prod. 237, 117689.

Rahman, G., Najaf, Z., Mehmood, A., Bilal, S., Shah, A.U., Mian, S.A., Ali, G., 2019b. An overview of the recent progress in the synthesis and applications of carbon nanotubes. Journal of Carbon Research 5, 1-31.

Rajendran, K., Karunagaran, V., Mahanty, B., Sen, S., 2015. Biosynthesis of hematite nanoparticles and its cytotoxic effect on HepG2 cancer cells. Int. J. Biol. Macromol. 74, 376-381.

Rajendran, K., Sen, S., 2018. Adsorptive removal of carbamazepine using biosynthesized hematite nanoparticles. Environ. Nanotechnology, Monit. Manag. 9, 122-127.

Rawtani, D., Khatri, N., Tyagi, S., Pandey, G., 2018. Nanotechnology-based recent approaches for sensing and remediation of pesticides. J. Environ. Manag. 206 749-762.

Redlich, O., Peterson, D.L., 1959. A useful adsorption isotherm. J. Phys. Chem. 63, 1024-1026.

Rodriguez-Mozaz, S., Ricart, M., Köck-Schulmeyer, M., Guasch, H., Bonnineau, C. Proia, L., de Alda, M.L., Sabater, S., Barceló, D., 2015. Pharmaceuticals and pesticides in reclaimed water: efficiency assessment of a microfiltration-reverse osmosis (MF-RO) pilot plant. J. Hazard Mater. 282, 165-173.

Rolph, C.A., Jefferson, B., Hassard, F., Villa, R., 2018. Metaldehyde removal from drinking water by adsorption onto filtration media: mechanisms and optimisation. Environ. Sci. Water Res. Technol. 4, 1543-1552.

Rostamian, R., Behnejad, H., 2018. A comprehensive adsorption study and modeling of antibiotics as a pharmaceutical waste by graphene oxide nanosheets. Ecotoxicol. Environ. Saf. 147, 117-123.

Saleh, I.A., Zouari, N., Al-Ghouti, M.A., 2020. Removal of pesticides from water and wastewater: chemical, physical and biological treatment approaches. Environ. Technol. Innov. 19, 101026.

Sarkar, B., Mandal, S., Tsang, Y.F., Kumar, P., Kim, K.H., Ok, Y.S., 2018. Designer carbon nanotubes for contaminant removal in water and wastewater: a critical review. Sci. Total Environ. 612, 561-581.

Schneider, T., Westermann, M., Glei, M., 2017. In vitro uptake and toxicity studies of metal nanoparticles and metal oxide nanoparticles in human HT29 cells. Arch. Toxicol. 91, 3517-3527.

Schug, T.T., Janesick, A., Blumberg, B., Heindel, J.J., 2011. Endocrine disrupting chemicals and disease susceptibility. J. Steroid Biochem. Mol. Biol. 127, 204-215.

Schultz, J., Capobianco, G., da Silva Veiga, P.A., Fornari, M.R., Antonangelo, A.R. Tebcherani, S.M., Mangrich, A.S., Pianaro, S.A., 2020. Sustainable activated carbon obtained as a by-product of the sugar and alcohol industry for removal of amoxicillin from aqueous solution. Energy, Ecol. Environ 5, 433-443. https:// doi.org/10.1007/s40974-020-00173-3.

Selvaraj, M., Hai, A., Banat, F., Haija, M.A., 2020. Application and prospects of carbon nanostructured materials in water treatment: a review. J. Water Process Eng. 33 100996.

Sengul, A.B., Asmatulu, E., 2020. Toxicity of metal and metal oxide nanoparticles: a review. Environ. Chem. Lett. 18, 1659-1683.

Seo, J., Lee, Y.G., Kim, S.D., Cha, C.J., Ahn, J.H., Hur, H.G., 2005. Biodegradation of the insecticide $\mathrm{N}, \mathrm{N}$-diethyl-m-toluamide by fungi: identification and toxicity of metabolites. Arch. Environ. Contam. Toxicol. 48, 323-328.

Shaker, M.A., Yakout, A.A., 2016. Optimization, isotherm, kinetic and thermodynamic studies of $\mathrm{Pb}$ (II) ions adsorption onto $\mathrm{N}$-maleated chitosan-immobilized $\mathrm{TiO}_{2}$ nanoparticles from aqueous media. Spectrochim. Acta Part A Mol. Biomol. Spectrosc. 154, 145-156.

Shan, D., Deng, S., Li, J., Wang, H., He, C., Cagnetta, G., Wang, B., Wang, Y., Huang, J. Yu, G., 2017. Preparation of porous graphene oxide by chemically intercalating a rigid molecule for enhanced removal of typical pharmaceuticals. Carbon N. Y. 119, 101-109.

Shanmugham, S.R., Jegadeesan, G.B., Ponnusami, V., 2020. Chapter 2 - groundwater treatments using nanomaterials. In: Micro and Nano Technologies. Elsevier, pp. 25-49.

Shareena, T.P.D., McShan, D., Dasmahapatra, A.K., Tchounwou, P.B., 2018. A review on graphene-based nanomaterials in biomedical applications and risks in environment and health. Nano-Micro Lett. 10, 1-34.

Sharma, L., Kakkar, R., 2018. Magnetically retrievable one-pot fabrication of mesoporous magnesium ferrite $\left(\mathrm{MgFe}_{2} \mathrm{O}_{4}\right)$ for the remediation of chlorpyrifos and real pesticide wastewater. J. Environ. Chem. Eng. 6, 6891-6903.

Sharma, L., Kakkar, R., 2017. Hierarchical porous magnesium oxide (Hr-MgO) microspheres for adsorption of an organophosphate pesticide: kinetics, isotherm, thermodynamics, and DFT studies. ACS Appl. Mater. Interfaces 9, 38629-38642.

Sharma, N., Dhiman, N., 2017. Kinetic and thermodynamic studies for ciprofloxacin hydrochloride adsorption from aqueous solution on CuO nanoparticles. Int. J. Chem. Res. 10, 98-106.

Shejale, K.P. Yadav, D. Patil, H., Saxena, S., Shukla, S., 2020. Evaluation of technique for the remediation of antibiotic-contaminated water using activated carbon. Mol. Syst. Des. Eng. 5, 743-756.

Sherer, T.B., Richardson, J.R., Testa, C.M., Seo, B.B., Panov, A.V., Yagi, T., MatsunoYagi, A., Miller, G.W., Greenamyre, J.T., 2007. Mechanism of toxicity of pesticides acting at complex I: relevance to environmental etiologies of Parkinson's disease. J. Neurochem. 100, 1469-1479.

Shokry, H., Elkady, M., Hamad, H., 2019. Nano activated carbon from industrial mine coal as adsorbents for removal of dye from simulated textile wastewater: operational parameters and mechanism study. J. Mater. Res. Technol. 8 $4477-4488$

Shrivas, K., Patel, S., Maji, P., Sinha, D., 2019. In: Abd-Elsalam, K.A., Mohamed, M.A. Prasad, R. (Eds.), Application of Magnetic Nanoparticles for Removal of Pesticides from Environmental Samples Prior to Instrumental Analysis BT - Magnetic Nanostructures : Environmental and Agricultural Applications. Springer International Publishing, Cham, pp. 247-260.

Singh, N.B., Nagpal, G., Agrawal, S., Rachna, 2018. Water purification by using adsorbents: a review. Environ. Technol. Innov. 11, 187-240.

Sips, R., 1948. Combined form of Langmuir and Freundlich equations. J. Chem. Phys. 16, 490-495.

Sobhan, A., Muthukumarappan, K., Cen, Z., Wei, L., 2019. Characterization of 
nanocellulose and activated carbon nanocomposite films' biosensing properties for smart packaging. Carbohydr. Polym. 225, 115189.

Spaltro, A., Pila, M., Simonetti, S., Álvarez-Torrellas, S., Rodríguez, J.G., Ruiz, D., Compañy, A.D., Juan, A., Allegretti, P., 2018. Adsorption and removal of phenoxy acetic herbicides from water by using commercial activated carbons: experimental and computational studies. J. Contam. Hydrol. 218, 84-93.

Srivastava, V., Zare, E.N., Makvandi, P., Zheng, X., Iftekhar, S., Wu, A., Padil, V.V.T., Mokhtari, B., Varma, R.S., Tay, F.R., 2020. Cytotoxic aquatic pollutants and their removal by nanocomposite-based sorbents. Chemosphere 258, 127324.

Suo, F., Liu, X., Li, C., Yuan, M., Zhang, B., Wang, J., Ma, Y., Lai, Z., Ji, M., 2019. Mesoporous activated carbon from starch for superior rapid pesticides removal. Int. J. Biol. Macromol. 121, 806-813.

Tang, L., Ma, X.Y., Wang, Y., Zhang, S., Zheng, K., Wang, X.C., Lin, Y., 2020. Removal of trace organic pollutants (pharmaceuticals and pesticides) and reduction of biological effects from secondary effluent by typical granular activated carbon. Sci. Total Environ. 749, 141611.

Tarcan, R., Todor-Boer, O., Petrovai, I., Leordean, C., Astilean, S., Botiz, I., 2020. Reduced graphene oxide today. J. Mater. Chem. C 8, 1198-1224.

Teixeira, S., Delerue-Matos, C., Santos, L., 2019. Application of experimental design methodology to optimize antibiotics removal by walnut shell based activated carbon. Sci. Total Environ. 646, 168-176.

Temkin, M.J., Pyzhev, V., 1940. Kinetics of ammonia synthesis on promoted ironcatalysts. Acta Physicochim 12, 217-222.

Thangavel, S., Venugopal, G., 2014. Understanding the adsorption property of graphene-oxide with different degrees of oxidation levels. Powder Technol. 257, $141-148$.

Tong, Y., McNamara, P.J., Mayer, B.K., 2019. Adsorption of organic micropollutants onto biochar: a review of relevant kinetics, mechanisms and equilibrium. Environ. Sci. Water Res. Technol. 5, 821-838.

Touraud, E., Roig, B., Sumpter, J.P., Coetsier, C., 2011. Drug residues and endocrine disruptors in drinking water: risk for humans? Int. J. Hyg Environ. Health 214, 437-441.

Tovar, A.K., Godínez, L.A., Espejel, F., Ramírez-Zamora, R.M., Robles, I., 2019. Optimization of the integral valorization process for orange peel waste using a design of experiments approach: production of high-quality pectin and activated carbon. Waste Manag. 85, 202-213.

Umbreen, N., Sohni, S., Ahmad, I., Khattak, N.U., Gul, K., 2018. Self-assembled threedimensional reduced graphene oxide-based hydrogel for highly efficient and facile removal of pharmaceutical compounds from aqueous solution. J. Colloid Interface Sci. 527, 356-367.

Varma, R.S., 2019. Biomass-derived renewable carbonaceous materials for sustainable chemical and environmental applications. ACS Sustain. Chem. Eng. 7, 6458-6470.

Veclani, D., Melchior, A., 2020. Adsorption of ciprofloxacin on carbon nanotubes: insights from molecular dynamics simulations. J. Mol. Liq. 298, 111977.

Vijaya Bhaskar Reddy, A., Moniruzzaman, M., Veera Manohara Reddy, Y., Madhavi, G., 2019. Chapter 18 - graphene-based nanomaterials for the removal of pharmaceuticals in drinking water sources. In: Micro and Nano Technologies. Elsevier, pp. 329-358.

Viotti, P.V., Moreira, W.M., Santos, O.A.A. dos, Bergamasco, R., Vieira, A.M.S., Vieira, M.F. 2019. Diclofenac removal from water by adsorption on Moringa oleifera pods and activated carbon: mechanism, kinetic and equilibrium study. J. Clean. Prod. 219, 809-817.

Völker, J., Stapf, M., Miehe, U., Wagner, M., 2019. Systematic review of toxicity removal by advanced wastewater treatment technologies via ozonation and activated carbon. Environ. Sci. Technol. 53, 7215-7233.

Wallace, P.R., 1947. The band theory of graphite. Phys. Rev. 71, 622-634.

Wang, C.Y., Makvandi, P., Zare, E.N., Tay, F.R., Niu, L.N., 2020a. Advances in antimicrobial organic and inorganic nanocompounds in biomedicine. Adv. Ther. 3, 2000024.

Wang, F., Ma, S., Si, Y., Dong, L., Wang, X., Yao, J., Chen, H., Yi, Z., Yao, W., Xing, B., 2017. Interaction mechanisms of antibiotic sulfamethoxazole with various graphene-based materials and multiwall carbon nanotubes and the effect of humic acid in water. Carbon N. Y. 114, 671-678.

Wang, L., Shi, C., Wang, L., Pan, L., Zhang, X., Zou, J.J., 2020b. Rational design, synthesis, adsorption principles and applications of metal oxide adsorbents: a review. Nanoscale 12, 4790-4815.

Wang, T., Zhang, Z., Zhang, H., Zhong, X., Liu, Y., Liao, S., Yue, X., Zhou, G., 2019a. Sorption of carbendazim on activated carbons derived from rape straw and its mechanism. RSC Adv. 9, 41745-41754.

Wang, Y., Huang, H., Wei, X., 2018. Influence of wastewater precoagulation on adsorptive filtration of pharmaceutical and personal care products by carbon nanotube membranes. Chem. Eng. J. 333, 66-75.

Wang, Y., Pan, C., Chu, W., Vipin, A.K., Sun, L., 2019b. Environmental remediation applications of carbon nanotubes and graphene oxide: adsorption and catalysis, Nanomaterials 9, 439.

Wang, Y., Wang, S., Xie, T., Cao, J., 2020c. Activated carbon derived from waste tangerine seed for the high-performance adsorption of carbamate pesticides from water and plant. Bioresour. Technol. 316, 123929.

Wang, Z., Gao, Z., Feng, S., Wang, J., Guo, X., 2019c. Experimental and computational study on the adsorption mechanism of 2-arylpropionic acids on graphene: solvent effects and aromatic features affecting the adsorption performance. Ind Eng. Chem. Res. 58, 8072-8079.

Watkins, M., Sizochenko, N., Moore, Q., Golebiowski, M., Leszczynska, D. Leszczynski, J., 2017. Chlorophenol sorption on multi-walled carbon nanotubes: DFT modeling and structure-property relationship analysis. J. Mol. Model. 23 39.

Weber, W.J., Morris, J.C., 1963. Kinetics of adsorption on carbon from solution. J. Sanit. Eng. Div. 89, 31-60.

Wei, X., Wu, Z., Wu, Z., Ye, B.-C., 2018. Adsorption behaviors of atrazine and Cr(III) onto different activated carbons in single and co-solute systems. Powder Technol. 329, 207-216.

Wernke, G., Shimabuku-Biadola, Q.L., dos Santos, T.R.T., Silva, M.F., FagundesKlen, M.R., Bergamasco, R., 2020. Adsorption of cephalexin in aqueous media by graphene oxide: kinetics, isotherm, and thermodynamics. Environ. Sci. Pollut. Res. 27, 4725-4736.

Wolfram, J., Stehle, S., Bub, S., Petschick, L.L., Schulz, R., 2019. Insecticide risk in US surface waters: drivers and spatiotemporal modeling. Environ. Sci. Technol. 53, 12071-12080.

Wu, F.C., Tseng, R.L., Juang, R.S., 2009. Characteristics of Elovich equation used for the analysis of adsorption kinetics in dye-chitosan systems. Chem. Eng. J. 150, 366-373.

Wu, T., Xue, Q., Liu, F., Zhang, J., Zhou, C., Cao, J., Chen, H., 2019. Mechanistic insight into interactions between tetracycline and two iron oxide minerals with different crystal structures. Chem. Eng. J. 366, 577-586.

Wu, W., Jiang, C.Z., Roy, V.A.L., 2016. Designed synthesis and surface engineering strategies of magnetic iron oxide nanoparticles for biomedical applications. Nanoscale 8, 19421-19474.

Xiang, Y., Xu, Z., Wei, Y., Zhou, Y., Yang, X., Yang, Y., Yang, J., Zhang, J., Luo, L., Zhou, Z. 2019. Carbon-based materials as adsorbent for antibiotics removal: mechanisms and influencing factors. J. Environ. Manag. 237, 128-138.

Yadav, S., Goel, N., Kumar, V., Singhal, S., 2019. Graphene oxide as proficien adsorbent for the removal of harmful pesticides: comprehensive experimental cum DFT investigations. Anal. Chem. Lett. 9, 291-310.

Yadav, S., Goel, N., Kumar, V., Tikoo, K., Singhal, S., 2018. Removal of fluoroquinolone from aqueous solution using graphene oxide: experimental and computational elucidation. Environ. Sci. Pollut. Res. 25, 2942-2957.

Yan, F., Kumar, S., Spyrou, K., Syari'ati, A., De Luca, O., Thomou, E., Alfonsín, E.M., Gournis, D., Rudolf, P., 2020. Highly Efficient Remediation of Chloridazon and its Metabolites: the Case of Graphene Oxide Nanoplatelets. ACS ES\&T Water.

Yang, B., Liu, Y., Liang, Q., Chen, M., Ma, L., Li, L., Liu, Q., Tu, W., Lan, D., Chen, Y. 2019a. Evaluation of activated carbon synthesized by one-stage and two-stage co-pyrolysis from sludge and coconut shell. Ecotoxicol. Environ. Saf. 170 $722-731$.

Yang, M., Yuan, Z., Liu, J., Fang, Z., Fang, L., Yu, D., Li, Q., 2019b. Photoresponsive actuators built from carbon-based soft materials. Adv. Opt. Mater. 7, 1900069.

Yek, P.N.Y., Liew, R.K., Osman, M.S., Lee, C.L., Chuah, J.H., Park, Y.K., Lam, S.S., 2019 Microwave steam activation, an innovative pyrolysis approach to convert waste palm shell into highly microporous activated carbon. J. Environ. Manag. 236, 245-253.

Yi, P., Zhang, D., Qiu, D., Peng, L., Lai, X., 2019. Carbon-based coatings for metallic bipolar plates used in proton exchange membrane fuel cells. Int. J. Hydrogen Energy 44, 6813-6843.

Yin, R., Sun, J., Xiang, Y., Shang, C., 2018. Recycling and reuse of rusted iron particles containing core-shell Fe-FeOOH for ibuprofen removal: adsorption and persulfate-based advanced oxidation. J. Clean. Prod. 178, 441-448.

Yu, C., Bahashi, J., Bi, E., 2019. Mechanisms and quantification of adsorption of three anti-inflammatory pharmaceuticals onto goethite with/without surface-bound organic acids. Chemosphere 222, 593-602.

Yusuf, M., Elfohi, F.M., Zaidi, S.A., Abdullah, E.C., Khan, M.A., 2015. Applications of graphene and its derivatives as an adsorbent for heavy metal and dye removal: a systematic and comprehensive overview. RSC Adv. 5, 50392-50420.

Yusuf, M., Khan, M.A., Abdullah, E.C. Elfohi, M., Hosomi, M., Terada, A., Riya, S. Ahmad, A., 2016. Dodecyl sulfate chain anchored mesoporous graphene: synthesis and application to sequester heavy metal ions from aqueous phase. Chem. Eng. J. 304, 431-439.

Yusuf, M., Khan, M.A., Otero, M., Abdullah, E.C., Hosomi, M., Terada, A., Riya, S., 2017. Synthesis of CTAB intercalated graphene and its application for the adsorption of AR265 and AO7 dyes from water. J. Colloid Interface Sci. 493, 51-61.

Yusuf, M., Kumar, M., Khan, M.A., Sillanpää, M., Arafat, H., 2019. A review on exfoliation, characterization, environmental and energy applications of graphene and graphene-based composites. Adv. Colloid Interface Sci. 273, 102036

Zare, EN . Motahari, A Sillanpää, M., 2018. Nanoadsorbents based on conducting polymer nanocomposites with main focus on polyaniline and its derivatives for removal of heavy metal ions/dyes: a review. Environ. Res. 162, 173-195.

Zareei, F., Ghoreyshi, A.A., 2011. Modeling of air stripping-vapor permeation hybrid process for removal of Vocs from wastewater and VOCs recovery. World Appl. Sci. J. 13, 2067-2074.

Zhang, S., Gu, P., Ma, R., Luo, C., Wen, T., Zhao, G., Cheng, W., Wang, X., 2019. Recent developments in fabrication and structure regulation of visible-light-driven $\mathrm{g}$ $\mathrm{C}_{3} \mathrm{~N}_{4}$-based photocatalysts towards water purification: a critical review. Catal. Today 335, 65-77. 
Zhang, W., 2003. Nanoscale iron particles for environmental remediation: an overview. J. Nanoparticle Res. 5, 323-332.

Zhao, D.L., Chung, T.S., 2018. Applications of carbon quantum dots (CQDs) in membrane technologies: a review. Water Res. 147, 43-49.

Zhao, Y., Cho, C.W., Wang, D., Choi, J.W., Lin, S., Yun, Y.S., 2020. Simultaneous scavenging of persistent pharmaceuticals with different charges by activated carbon fiber from aqueous environments. Chemosphere 247, 125909.

Zhao, Y., Liu, F., Qin, X., 2017. Adsorption of diclofenac onto goethite: adsorption kinetics and effects of pH. Chemosphere 180, 373-378.

Zhou, Y., He, Y., Xiang, Y., Meng, S., Liu, X., Yu, J., Yang, J., Zhang J., Oin, P., Luo, L. 2019. Single and simultaneous adsorption of pefloxacin and $\mathrm{Cu}(\mathrm{II})$ ions from aqueous solutions by oxidized multiwalled carbon nanotube. Sci. Total Environ. 646, 29-36.

Zhu, Y., Wu, J., Chen, M., Liu, X., Xiong, Y., Wang, Y., Feng, T., Kang, S., Wang, X., 2019. Recent advances in the biotoxicity of metal oxide nanoparticles: impacts on plants, animals and microorganisms. Chemosphere 237, 124403. 


\section{Supplementary Material}

\section{Toxicity and remediation of pharmaceuticals and pesticides using metal oxides and carbon nanomaterials}

Zari Fallah ${ }^{1}$, Ehsan Nazarzadeh Zare ${ }^{2 *}$, Matineh $\mathrm{Ghomi}^{2}$, Farhad Ahmadijokani ${ }^{3}$, Majed Amini ${ }^{3}$, Mahmood Tajbakhsh ${ }^{1}$, Mohammad Arjmand ${ }^{3}$, Gaurav Sharma ${ }^{4}$, Humna Ali ${ }^{5}$, Awais Ahmad $^{5}$, Pooyan Makvandi ${ }^{6}$, Eric Lichtfouse ${ }^{7 *}$, Mika Sillanpää8, ${ }^{8,9}$ Rajender Varma ${ }^{10,11 *}$

${ }^{1}$ Faculty of Chemistry, University of Mazandaran, Babolsar, 47416-95447, Iran

${ }^{2}$ School of Chemistry, Damghan University, Damghan 36716-41167, Iran

${ }^{3}$ School of Engineering, University of British Columbia, Kelowna, BC, V1V 1V7, Canada

${ }^{4}$ College of Materials Science and Engineering, Shenzhen Key Laboratory of Polymer Science and Technology, Guangdong Research Center for Interfacial Engineering of Functional Materials, Nanshan District Key Lab. for Biopolymers and Safety Evaluation, Shenzhen University, Shenzhen, 518055, PR China

${ }^{5}$ Department of Chemistry, The University of Lahore, Lahore 54590, Pakistan

${ }^{6}$ Center for Micro-BioRobotics, Istituto Italiano di Tecnologia (IIT), Viale R. Piaggio 34, 56025 Pontedera, Pisa, Italy

${ }^{7}$ Aix-Marseille University, CNRS, IRD, INRA, Coll France, CEREGE, 13100 Aix en Provence, France. Orcid: 0000-0002-8535-8073

${ }^{8}$ Institute of Research and Development, Duy Tan University, Da Nang 550000, Vietnam.

Faculty of Environment and Chemical Engineering, Duy Tan University, Da Nang 550000, Vietnam.

${ }^{9}$ Department of Chemical Engineering, School of Mining, Metallurgy and Chemical Engineering, University of Johannesburg, P. O. Box 17011, Doornfontein 2028, South Africa

${ }^{10}$ Chemical Methods and Treatment Branch, Water Infrastructure Division, Center for Environmental Solutions and Emergency Response, U. S. Environmental Protection Agency, 26 West Martin Luther King Drive, Cincinnati, Ohio 45268, USA

${ }^{11}$ Regional Center of Advanced Technologies and Materials, Department of Physical Chemistry, Faculty of Science, Palacky University, Š lechtitelů 27, 783 71, Olomouc, Czech Republic

Coresponding authors-Email: ehsan.nazarzadehzare@gmail.com (E.N.Zare); eric.lichtfouse@inrae.fr (E. Lichtfouse); Varma.Rajender@epa.gov (R.Varma) 


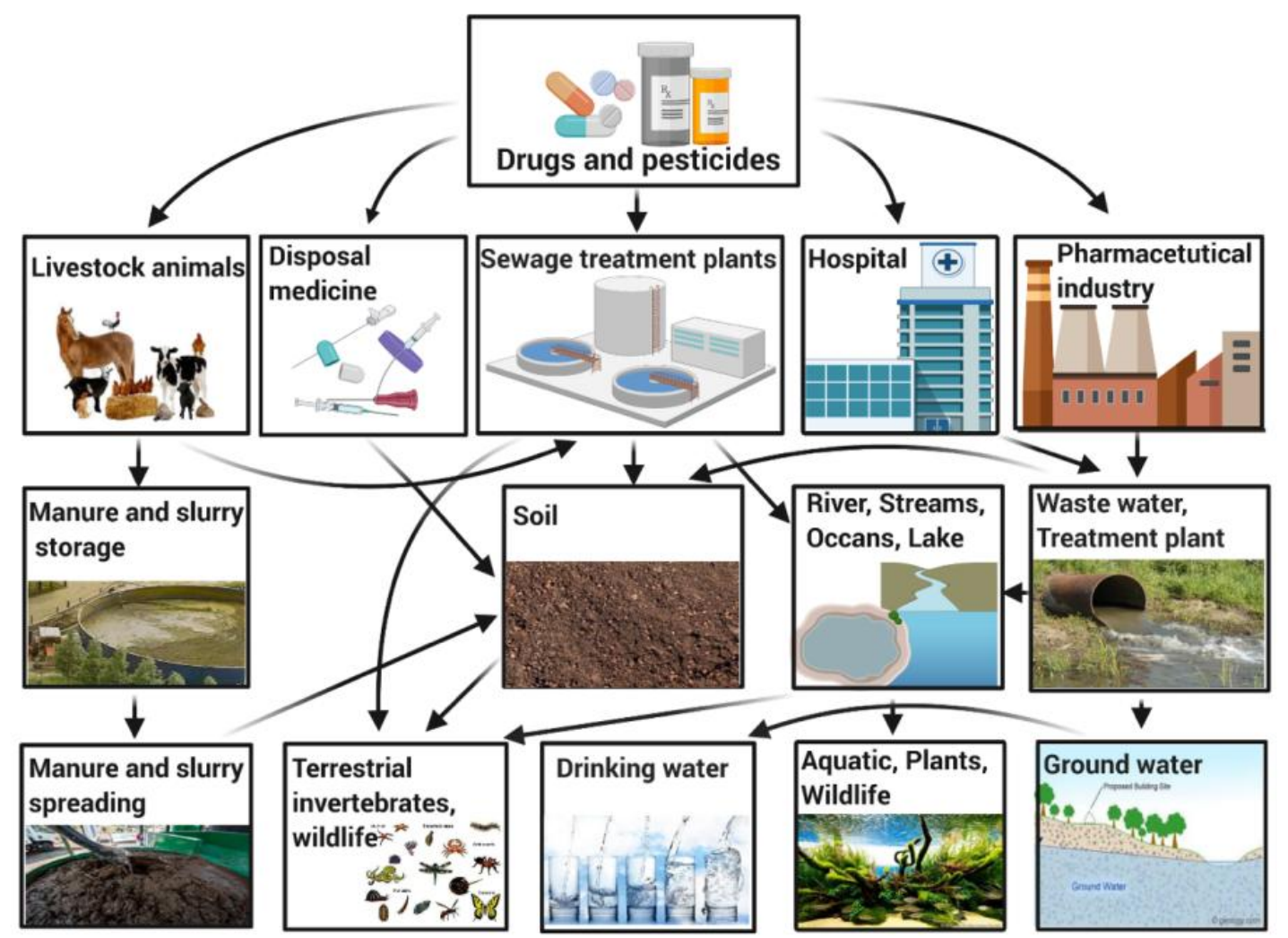

Fig. 1S. Major sources and fate of pharmaceuticals and pesticides. Reprinted with permission (Kar et al., 2020). 


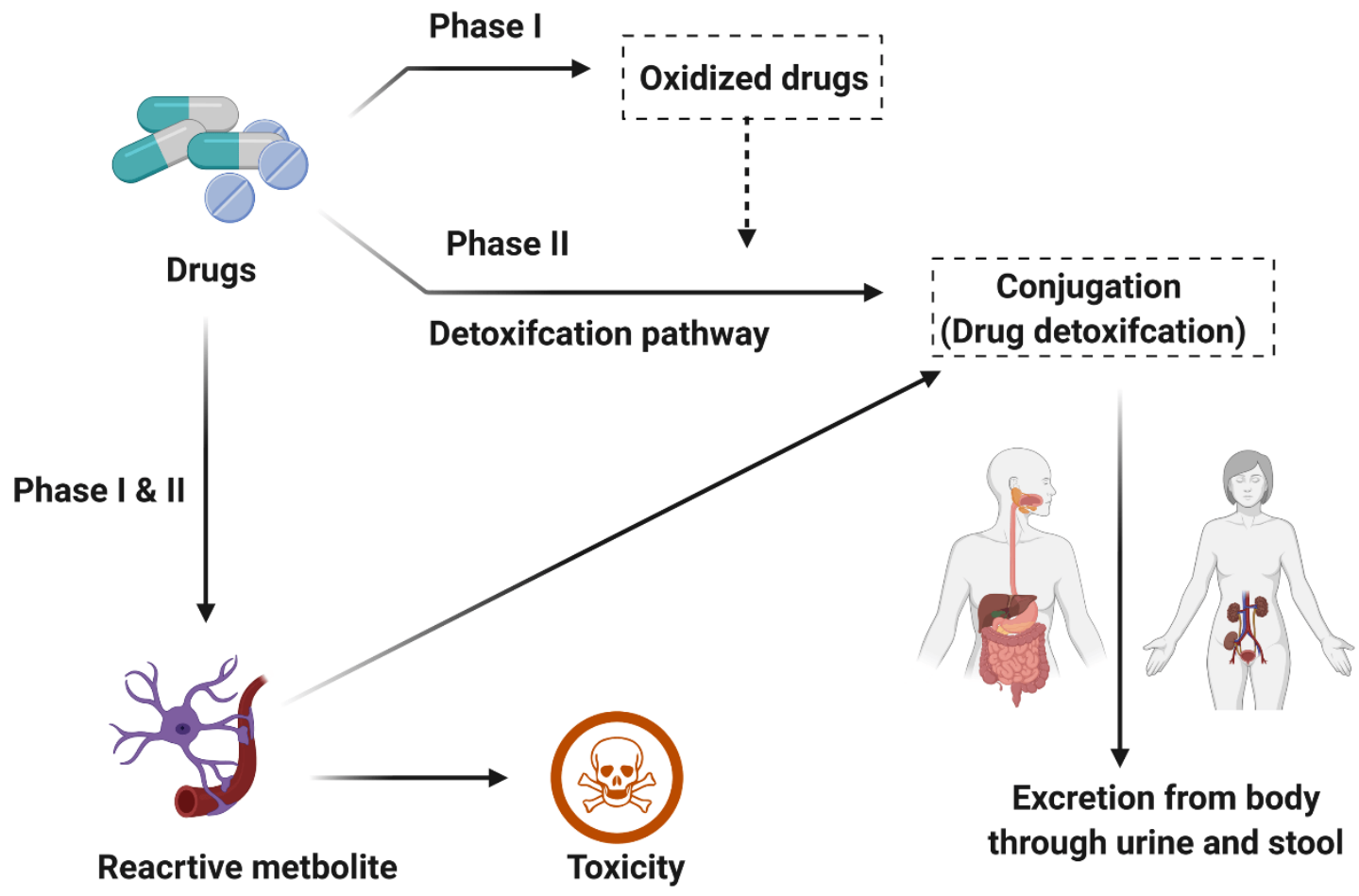

Fig. 2S. General pathways of drug metabolism. CYP450: Cytochrome P450. Reproduced with permission from (Jaladanki et al., 2020). 


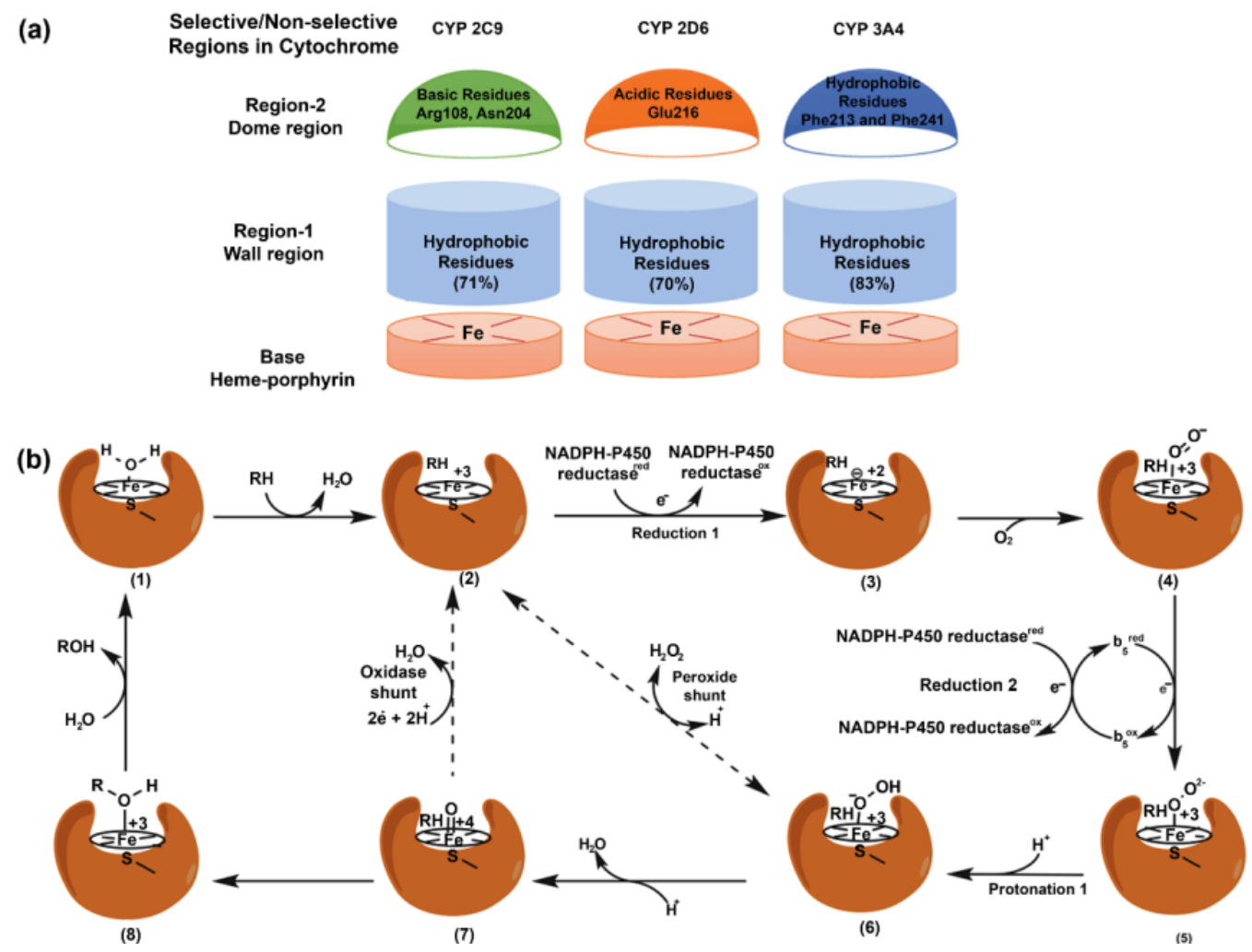

Fig. 3S. (a) Catalytic site for cytochromes and (b) the generalized catalytic cycle of a cytochrome P450 enzyme. Three regions of catalytic site for cytochromes are stated as base, wall, and dome. The location of the drug inside the cavity is determined by the chemical characteristics of these regions. The dome region plays a major role in this regard so that hydrophobic groups with a viewpoint of metabolism are drawn to the Fe center. CYP: cytochromes; NADPH: nicotinamide adenine dinucleotide phosphate (reduced form); RH: drug. Reprinted with permission from (Jaladanki et al., 2020). 
(a)

1. Oxidation by CYPs

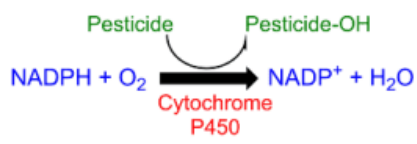

2. Futile cycling

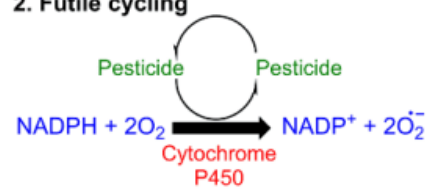

(b)
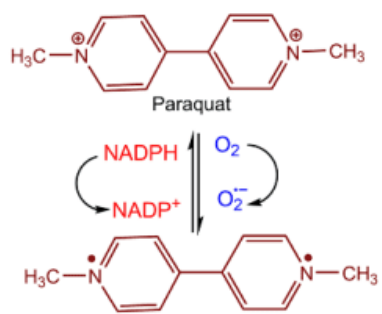

(c)

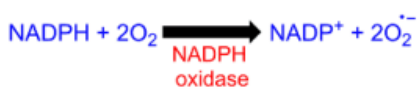

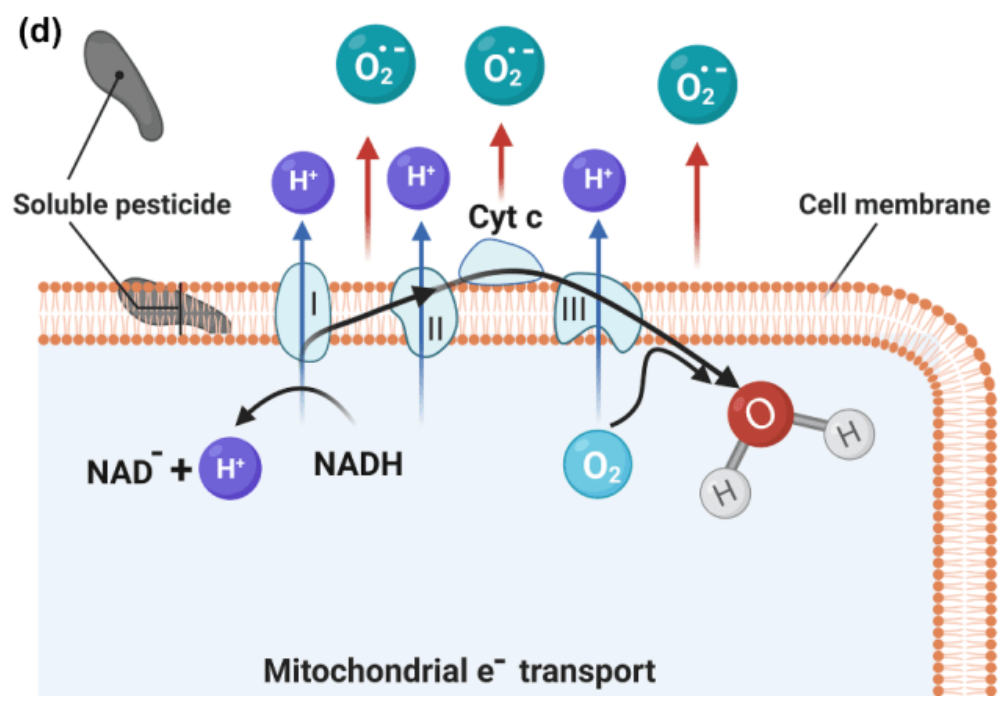

Fig. 4S. The equations of pesticide oxidation catalyzed by cytochrome P450 $(a, b)$ Normal oxidation cause and inefficacious cycling for superoxides production; $(\mathrm{c}, \mathrm{d})$ useless oxidative metabolism of a pesticide through CYP450s, create an uncoupling reaction and superoxide generation. CYP450: Cytochrome P450. Reprinted with modification with permission from (Gray et al., 2013). 


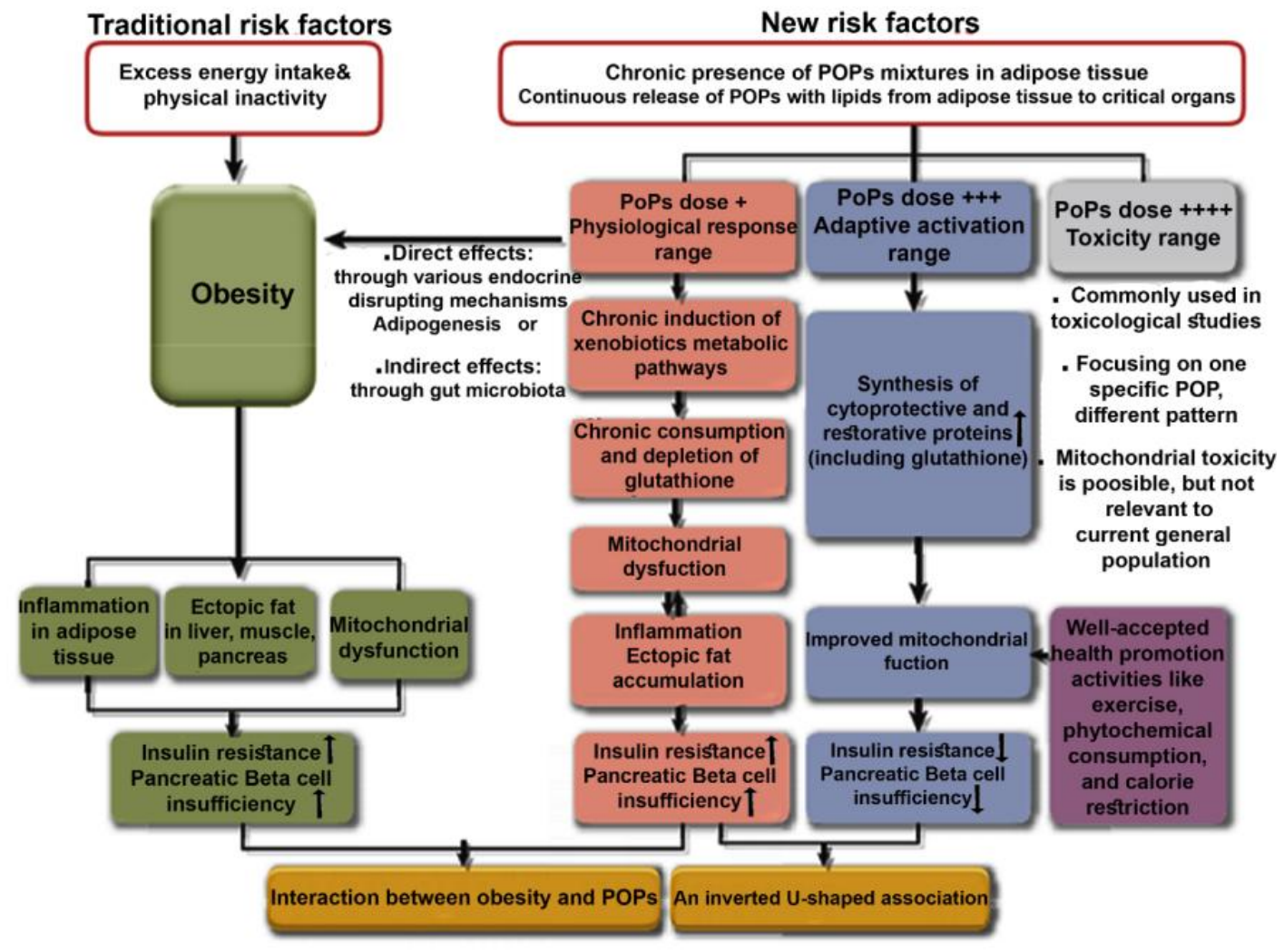

Fig. 5S. Summary of postulated relationships between persistent organic pollutants (POP) and their relationships on the risk of type 2 diabetes $\left(T_{2} D\right)$. Reprinted with permission from(Lee et al., 2014). 
Table 1S. Human health risk ratios and acceptable daily intake of some reported drugs.(Chinnaiyan et al., 2018; Cunningham et al., 2009)

\begin{tabular}{|c|c|c|c|c|c|}
\hline Therapeutic class of drugs & Generic name & $\begin{array}{c}\text { ADI }{ }^{\text {a) }} \\
{[\mu \mathrm{g} / \mathrm{kg} / \mathrm{day}]} \\
\end{array}$ & $\begin{array}{l}\text { PEC }^{\text {b) }} / \text { PNEC }^{\text {c) }} \\
\text { [North America] }\end{array}$ & $\begin{array}{c}\text { PEC / PNEC } \\
\text { [Europe] } \\
\end{array}$ & $\begin{array}{c}\text { PEC / PNEC } \\
\text { [India] } \\
\end{array}$ \\
\hline Anticancer & Topotecan hydrochloride & 0.0021 & $1.6 \mathrm{E}-04$ & $3.0 \mathrm{E}-04$ & - \\
\hline Anticancer & Mercaptopurine monohydrate & 0.0021 & $2.4 \mathrm{E}-02$ & $3.8 \mathrm{E}-02$ & - \\
\hline Anticancer & Vinorelbine & 0.0021 & $6.7 \mathrm{E}-04$ & $1.3 \mathrm{E}-03$ & - \\
\hline Anticancer & Melphalan hydrochloride & 0.0021 & $2.6 \mathrm{E}-04$ & $2.0 \mathrm{E}-04$ & - \\
\hline Benign prostatic hypertrophy & Dutasteride & 0.0016 & $2.9 \mathrm{E}-03$ & $2.3 \mathrm{E}-03$ & - \\
\hline Cardiovascular & Digoxin & 0.071 & $2.0 \mathrm{E}-03$ & $6.4 \mathrm{E}-04$ & - \\
\hline Antiviral (HIV) & Lamivudine & 15.9 & $1.8 \mathrm{E}-03$ & $5.0 \mathrm{E}-04$ & - \\
\hline Antiviral & Acyclovir/valacyclovir & 190 & $3.7 \mathrm{E}-04$ & $1.9 \mathrm{E}-04$ & - \\
\hline Antiviral & Abacavir hemisulfate & 57.1 & $7.6 \mathrm{E}-05$ & $2.4 \mathrm{E}-05$ & - \\
\hline Antiviral (HIV) & Amprenavir + fosamprenavir & 133 & 4.7E-06 & $1.3 \mathrm{E}-06$ & - \\
\hline Antiviral & Zanamavir & 3.14 & 8.9E-07 & 2.9E-06 & - \\
\hline Antiviral & Zidovudine & 14.3 & $1.5 \mathrm{E}-03$ & 4.3E-04 & - \\
\hline Antigastric acid & Ranitidine hydrochloride & 10.7 & $5.3 \mathrm{E}-03$ & $1.9 \mathrm{E}-02$ & - \\
\hline $\begin{array}{l}\text { Anti-inflammatory; } \\
\text { vasoconstrictor }\end{array}$ & Betamethasone valerate & 0.24 & $1.2 \mathrm{E}-03$ & $2.7 \mathrm{E}-03$ & - \\
\hline $\begin{array}{l}\text { Anti-inflammatory; } \\
\text { antipyretics }\end{array}$ & Acetyl salicylic acid & 87 & - & - & 0.07 \\
\hline Corticosteroids & Budesonide & 68 & - & - & - \\
\hline Antidepressant & Bupropion hydrochloride & 51.7 & $2.4 \mathrm{E}-04$ & $4.6 \mathrm{E}-06$ & - \\
\hline Antidiabetic & Metformin hydrochloride & 79.4 & $1.6 \mathrm{E}-02$ & $2.0 \mathrm{E}-02$ & - \\
\hline AntiParkinson's & Ropinirole hydrochloride & 0.12 & $1.2 \mathrm{E}-04$ & $7.5 \mathrm{E}-05$ & - \\
\hline Antimalarial & Atovaquone & 238 & $2.1 \mathrm{E}-06$ & $2.1 \mathrm{E}-05$ & - \\
\hline
\end{tabular}




\begin{tabular}{|c|c|c|c|c|c|}
\hline Antiasthma & Albuterol (salbutamol) & 0.05 & $6.1 \mathrm{E}-05$ & $2.6 \mathrm{E}-04$ & - \\
\hline Antidepressant & Fluoxetine & 77 & - & - & 0.06 \\
\hline Antidepressant & Amitriptyline & 166 & - & - & 0.13 \\
\hline
\end{tabular}

a) ADI: Acceptable daily intake; b) PEC: Predicted environmental concentration; and c) PNEC: Predicted no-effect concentration. 
Table 2S. Some pesticides and their health hazards.

\begin{tabular}{|c|c|c|c|}
\hline Compounds & Impact & Action mechanism & Reference \\
\hline 3,5,6-trichloro-2-pyridinol & Endocrine disruptors for androgen signaling & Reproductive disorders & $\begin{array}{l}\text { (Hazarika et } \\
\text { al., 2019) }\end{array}$ \\
\hline Terbufos (OP), Fonofos (OP) & $\begin{array}{l}\text { The significant interaction between exposure and } \\
\text { genetic variants, }\end{array}$ & Prostate cancer & $\begin{array}{l}\text { (Gray et al., } \\
2013)\end{array}$ \\
\hline Carbendazim & Possible Carcinogen, show Mutagen & $\begin{array}{l}\text { Human Ovarian Granulose-Like Tumor Cell Line } \\
(\mathrm{KGN})\end{array}$ & $\begin{array}{c}\text { (Morinaga et } \\
\text { al., 2004) }\end{array}$ \\
\hline Chlorantraniliprole & Possibly Eye irritant & Damage in the midgut of Bombyx mori & $\begin{array}{l}\text { (Bhandari et } \\
\text { al., 2019; Hu } \\
\text { et al., 2019) }\end{array}$ \\
\hline Chlorpyrifos & Neurotoxicant and psychiatric disorders & $\begin{array}{l}\text { Human liver hepatocellular carcinoma cells } \\
\text { (HepG2), neurotoxicity in rats }\end{array}$ & $\begin{array}{c}\text { (Mahmoud e } \\
\text { al., 2019; } \\
\text { Zhou and Li, } \\
\text { 2018) }\end{array}$ \\
\hline Dichlorvos & $\begin{array}{l}\text { Dyspnea, salivation, convulsion, and other } \\
\text { neurological phenomena }\end{array}$ & Chicken brain tissues, a gastric tissue cancer & $\begin{array}{c}\text { (Okoroiwu } \\
\text { and Iwara, } \\
\text { 2018; Xiao e } \\
\text { al., 2020) }\end{array}$ \\
\hline Dimethoate & Low dermal toxicity & Liver and brain of rats & $\begin{array}{l}\text { (Sharma et } \\
\text { al., 2005) }\end{array}$ \\
\hline Emamectin & $\begin{array}{l}\text { Apoptosis of QSG7701 cells and induced the DNA } \\
\text { damage }\end{array}$ & Human liver & $\begin{array}{c}\text { (Zhang et al., } \\
\text { 2017) } \\
\end{array}$ \\
\hline Imidacloprid & Genotoxicity & Liver & $\begin{array}{c}\text { Costa et al., } \\
2009 ; \\
\text { Schulz- } \\
\text { Jander et al., } \\
\text { 2002) }\end{array}$ \\
\hline Omethoate & Homozygous/heterozygous mutant genotypes & Alteration in the gene expression in the process of & (Duan et al., \\
\hline
\end{tabular}




\begin{tabular}{llr}
\hline Profenofos & $\begin{array}{c}\text { Neurotoxicant, skin irritant, and possibly eye } \\
\text { irritant }\end{array}$ & $\begin{array}{c}\text { (Bhandari et } \\
\text { al., 2019; } \\
\text { Moustafa et } \\
\text { al., 2007) }\end{array}$ \\
\hline Quinalphos & Neurotoxicant, skin and eye irritant & $\begin{array}{c}\text { (Bhandari et } \\
\text { al., 2019; } \\
\text { Govindarajan } \\
\text { et al., 2019) }\end{array}$ \\
\hline Triazophos & Neurotoxicant, skin and eye irritant & $\begin{array}{c}\text { Alzheimer's disease and male sterility in humans } \\
\text { (Bhandari et } \\
\text { al., 2019; } \\
\text { Jain et al., } \\
\text { 2011) }\end{array}$ \\
\hline
\end{tabular}




\section{References:}

Bhandari, G., Zomer, P., Atreya, K., Mol, H.G.J., Yang, X., Geissen, V., 2019. Pesticide residues in Nepalese vegetables and potential health risks. Environ. Res. 172, 511-521.

Chinnaiyan, P., Thampi, S.G., Kumar, M., Mini, K.M., 2018. Pharmaceutical products as emerging contaminant in water: relevance for developing nations and identification of critical compounds for Indian environment. Environ. Monit. Assess. 190, 288.

Costa, C., Silvari, V., Melchini, A., Catania, S., Heffron, J.J., Trovato, A., De Pasquale, R., 2009. Genotoxicity of imidacloprid in relation to metabolic activation and composition of the commercial product. Mutat. Res. Toxicol. Environ. Mutagen. 672, 40-44.

Cunningham, V.L., Binks, S.P., Olson, M.J., 2009. Human health risk assessment from the presence of human pharmaceuticals in the aquatic environment. Regul. Toxicol. Pharmacol. $53,39-45$.

Duan, X., Yang, Y., Wang, S., Feng, X., Wang, T., Wang, P., Liu, S., Li, L., Yao, W., Cui, L., 2017. Changes in the expression of genes involved in cell cycle regulation and the relative telomere length in the process of canceration induced by omethoate. Tumor Biol. 39, 1010428317719782.

Govindarajan, D., Chatterjee, C., Shakambari, G., Varalakshmi, P., Jayakumar, K., Balasubramaniem, A., 2019. Oxidative stress response, epigenetic and behavioral alterations in Caenorhabditis elegans exposed to organophosphorus pesticide quinalphos. Biocatal. Agric. Biotechnol. 17, 702-709.

Gray, J.W., Burns, C.J., Mahlburg, W.M., 2013. Increased cancer burden among pesticide applicators and others due to pesticide exposure. CA. Cancer J. Clin. 63, 364-366.

Hazarika, J., Ganguly, M., Mahanta, R., 2019. Molecular interactions of chlorpyrifos and its environmental degradation products with human sex hormone-binding globulin: an in silico study. J. Appl. Toxicol. 39, 1002-1011.

Hu, J., Li, M., Lu, Z., Mao, T., Chen, J., Wang, H., Qu, J., Fang, Y., Cheng, X., Li, J., 2019. The mechanism of damage in the midgut of Bombyx mori after chlorantraniliprole exposure. Ecotoxicology 28, 903-912.

Jain, S., Ahmed, R.S., Arora, V.K., Banerjee, B.D., 2011. Biochemical and histopathological studies to assess chronic toxicity of triazophos in blood, liver and brain tissue of rats. Pestic. Biochem. Physiol. 100, 182-186.

Jaladanki, C.K., Gahlawat, A., Rathod, G., Sandhu, H., Jahan, K., Bharatam, P. V, 2020. Mechanistic studies on the drug metabolism and toxicity originating from cytochromes P450. Drug Metab. Rev. 52, 366-394.

Kar, S., Sanderson, H., Roy, K., Benfenati, E., Leszczynski, J., 2020. Ecotoxicological assessment of pharmaceuticals and personal care products using predictive toxicology approaches. Green Chem. 22, 1458-1516.

Lee, D.-H., Porta, M., Jacobs Jr, D.R., Vandenberg, L.N., 2014. Chlorinated persistent organic pollutants, obesity, and type 2 diabetes. Endocr. Rev. 35, 557-601. 
Mahmoud, S.M., Moneim, A.E.A., Qayed, M.M., El-Yamany, N.A., 2019. Potential role of Nacetylcysteine on chlorpyrifos-induced neurotoxicity in rats. Environ. Sci. Pollut. Res. 26, 20731-20741.

Morinaga, H., Yanase, T., Nomura, M., Okabe, T., Goto, K., Harada, N., Nawata, H., 2004. A benzimidazole fungicide, benomyl, and its metabolite, carbendazim, induce aromatase activity in a human ovarian granulose-like tumor cell line (KGN). Endocrinology 145, 1860-1869.

Moustafa, G.G., Ibrahim, Z.S., Hashimoto, Y., Alkelch, A.M., Sakamoto, K.Q., Ishizuka, M., Fujita, S., 2007. Testicular toxicity of profenofos in matured male rats. Arch. Toxicol. 81, $875-881$.

Okoroiwu, H.U., Iwara, I.A., 2018. Dichlorvos toxicity: A public health perspective. Interdiscip. Toxicol. 11, 129-137.

Schulz-Jander, D.A., Leimkuehler, W.M., Casida, J.E., 2002. Neonicotinoid insecticides: reduction and cleavage of imidacloprid nitroimine substituent by liver microsomal and cytosolic enzymes. Chem. Res. Toxicol. 15, 1158-1165.

Sharma, Y., Bashir, S., Irshad, M., Nag, T.C., Dogra, T.D., 2005. Dimethoate-induced effects on antioxidant status of liver and brain of rats following subchronic exposure. Toxicology 215 , $173-181$.

Xiao, Y., Zheng, X., Li, G., Zhou, C., Wu, C., Xu, Z., Hu, G., Guo, X., Li, L., Cao, H., 2020. Investigation of the effects of dichlorvos poisoning on AMPK signaling pathway in chicken brain tissues. Environ. Pollut. 261, 114109.

Zhang, Z., Zhao, X., Qin, X., 2017. Potential genotoxic and cytotoxicity of emamectin benzoate in human normal liver cells. Oncotarget $8,82185$.

Zhou, C., Li, X., 2018. Cytotoxicity of chlorpyrifos to human liver hepatocellular carcinoma cells: effects on mitochondrial membrane potential and intracellular free $\mathrm{Ca} 2+$. Toxin Rev. 37, 259-268. 